\title{
Novel targets for the development of drugs for Type 2 Diabetes Mellitus
}




\section{Thesis committee}

\section{Promotor}

Prof. Dr Ivonne M.C.M. Rietjens

Professor of Toxicology

Wageningen University \& Research

\section{Co-promotor}

Dr Jacques J.M. Vervoort

Associate professor at the Laboratory of Biochemistry

Wageningen University \& Research

\section{Other members}

Prof. Dr Huub F.J. Savelkoul, Wageningen University \& Research

Dr Guido J.E.J. Hooiveld, Wageningen University \& Research

Prof. Dr Renger F. Witkamp, Wageningen University \& Research

Prof. Dr Martin van den Berg, Utrecht University

This research was conducted under the auspices of the Graduate School VLAG (Advanced studies in Food Technology, Agrobiotechnology, Nutrition and Health Sciences) 


\title{
Novel targets for the development of drugs for Type 2 Diabetes Mellitus
}

\author{
Alexandros Sotiriou
}

\section{Thesis}

submitted in fulfilment of the requirements for the degree of doctor at Wageningen University

by the authority of Rector Magnificus

Prof. Dr A.P.J. Mol, in the presence of the

Thesis Committee appointed by the Academic Board

to be defended in public

on Wednesday $21^{\text {st }}$ of December 2016

at 11 a.m. in the Aula. 
Alexandros Sotiriou

Novel targets for the development of drugs for Type 2 Diabetes Mellitus

PhD thesis, Wageningen University \& Research, Wageningen, NL (2016)

With references, with summary in English

ISBN: 978-94-6343-030-2

DOI: http://dx.doi.org/10.18174/399762 


\section{Table of contents}

Chapter 1

General Introduction

Chapter 2

25

Correlation between activation of PPARy and resistin downregulation in a mouse adipocyte cell line by a series of thiazolidinediones

Chapter 3

Toll-like receptor 4 (TLR-4) signaling as a method for detecting resistin antagonism

Chapter 4

Resistin exposure of human omental adipocytes affects transcriptional regulation processes involved in cellular differentiation and maintenance

Chapter 5

Identification of Type 2 Diabetes-related biomarkers derived from an in vitro model of inflamed fat tissue

Chapter 6

Discussion and future perspectives

Chapter 7

Summary

Acknowledgements

About the author

List of publications

Overview of completed training activities 



\section{Chapter 1}

General introduction 


\section{Chapter 1}

\section{Introduction}

Type 2 Diabetes Mellitus (T2DM) is a modern metabolic disorder with increasing number of patients over the last decades, and at present even reaching pandemic levels (Wang et al., 2011). Hyperglycemia, insulin resistance and impaired insulin production are the characteristics of T2DM (Olokoba et al., 2012; Lazar, 2007; Ferrannini, 1992). Insulin resistance is the inability to execute efficient glucose uptake by the insulin sensitive organs. T2DM results in disequilibrium of glucose in the body and consequently a disequilibrium in energy and lipid homeostasis. The development of T2DM can be affected by genomic, environmental and behavioral risk factors (Olokoba et al., 2012; Chen et al., 2011).

The aim of the present thesis was to define novel targets for the possible development of drugs for T2DM, and to this end this first chapter presents an overview of insulin function and adipose tissue as the modes of action underlying the adverse effects in T2DM. The chapter also describes the possible drugs for treatment of T2DM, thiazolidinediones (TZDs) used so far, their mode of action and apparent issues that arose from their use pointing at the need for the development of other targets for drug development than TZDs.

\section{Insulin function}

Insulin influences synthesis of glycogen, fatty acids, proteins very likely all through transport of glucose. As a result insulin can influence cellular development including adipocytes differentiation. (Kahn and Flier, 2000; Jung and Choi, 2014). Insulin is produced by the $\beta$ cells which are located in the islets of Langerhans in the pancreas. Whenever there is an excess of glucose in the blood, $\beta$-cells release insulin. Initially insulin binds to the insulin receptor (IR). IR then phosphorylates insulin receptor substrate (IRS). The activation of IRS activates two signaling pathways. The first is the phosphatidylinositol 3- kinase (PI3K) $\mathrm{AKT} /$ protein kinase $\mathrm{B}(\mathrm{PKB})$ pathway that is related to the metabolic actions of insulin. More specifically, the phosphorylation of IRS activates PI3K, which in turn activates a second messenger, phosphatidylinositol-(3,4,5)-triphosphate, which subsequently activates several phosphatidylinositol-(3,4,5)-triphosphate-dependent serine/threonine kinases, including AKT/PKB. The last one activates the translocation of glucose transporter 4 to the plasma membrane, which increases the glucose uptake (Saltiel and Kahn, 2001). The second signaling pathway that is affected by IRS activation is the Ras-mitogen-activated protein kinase (MAPK) pathway that is related to the mitogenic and growth effects of insulin. The 


\section{General Introduction}

MAPK pathway leads to the activation of genes that promote cell growth, inflammation and atherosclerosis (Jung and Choi, 2014).

FFAs and their metabolites, such as acyl-coenzyme A (acyl-CoA), ceramides and diacylglycerol (DAG) can impair insulin signaling. These enzymes can activate several serine/threonine kinases, such as protein kinase $\mathrm{C}(\mathrm{PKC})$, inhibitory kinase $\mathrm{kB}(\mathrm{IKK} \beta)$ and nuclear factor kappa B (NF-kB) (Schinner et al., 2005). PKC, IKK $\beta$, NF-kB etc. can also be activated by inflammatory molecules such as tumor necrosis factor $\alpha$ (TNF- $\alpha$ ) and interleukin-6 (IL-6) (Delarue and Magnan, 2007). The activation of these kinases leads to the inactivation or inhibition of the key proteins of the insulin pathway. Additionally inhibition of insulin-stimulated IRS-1 (Insulin receptor substrate 1) phosphorylation (Petersen and Shulman, 2006) can lead to less GLUT4 translocation and finally lower glucose uptake (Figure 1).

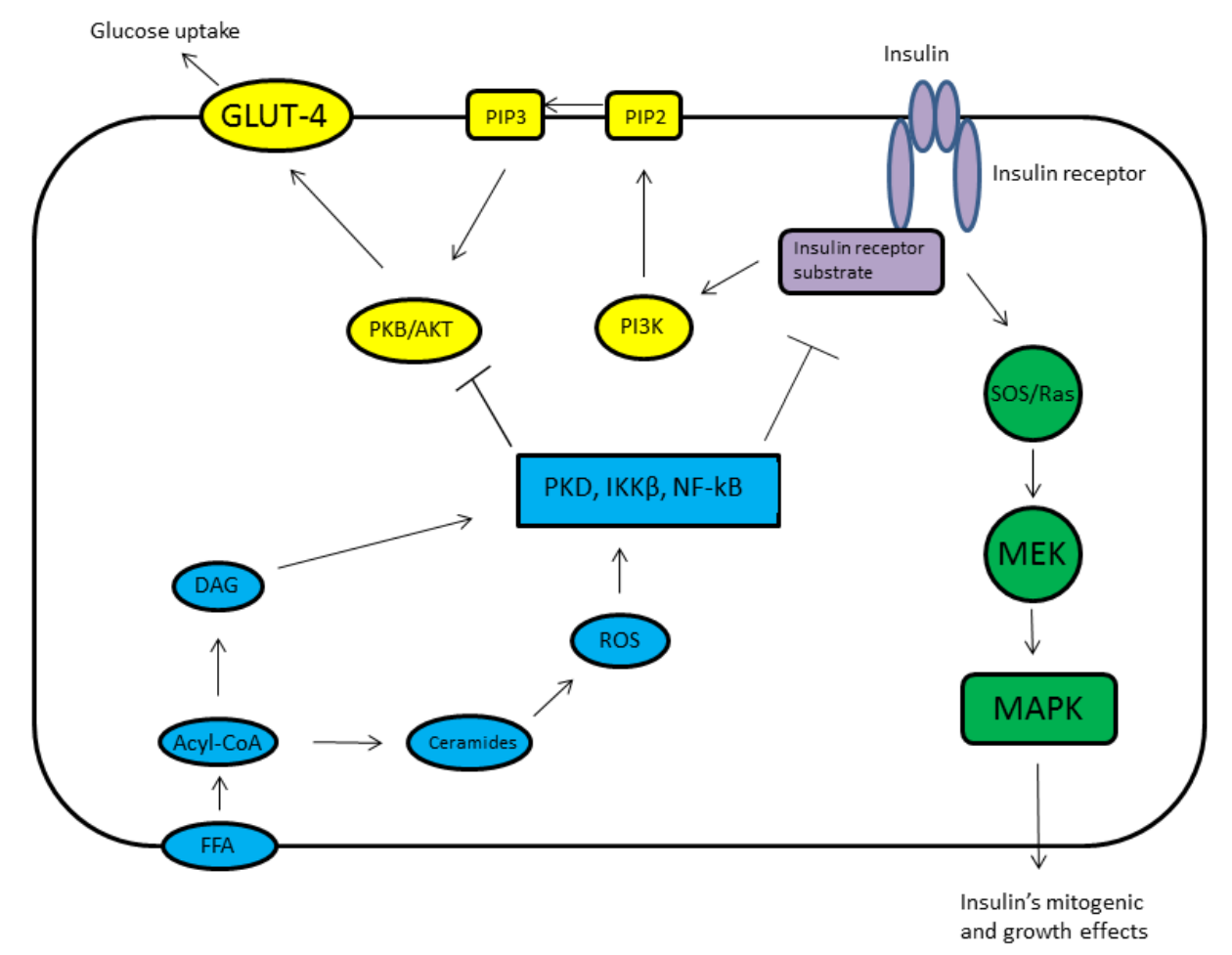

Figure 1. Schematic view of insulin pathways and inhibition by FFAs. Insulin binds to the insulin receptor. Then the insulin receptor phosphorylates the insulin receptor substrate (IRS) which in turn activates PI3K. Next PI3K activates PKB/AKT through the PIP2 and PIP3. Finally GLUT4 translocation is established and glucose uptake takes place. Parallel IRS activates MAPK through the activations of SOS/Ras and MEK. On the other hand FFA and its metabolites can inhibit insulin action by inhibition of insulin-stimulated IRS phosphorylation or inhibiting PKB/AKT with the PKD, IKK $\beta$ and NF-kB. PI3K, phosphatidylinositol 3 kinase; PKB/AKT, protein kinase B; GLUT-4, glucose transporter type 4; FFA, free fatty acids; DAG, diacylglycerol; ROS, reactive oxygen species; IKK $\beta$, inhibitory kB kinase $\beta$; NF-kB, nuclear factor kB. Based on Delarue and Magnan (2007) 


\section{Chapter 1}

\section{Adipose tissue function}

Adipose tissue plays a major role in the energy storage of the body and in energy homeostasis. In addition, adipose tissue is nowadays considered to be an endocrine organ that synthesizes and secretes biologically active substances (Ahima and Flier, 2000; Kershaw and Flier, 2004; Galic et al., 2010; Boden 2011). Adipose tissue consists of different kind of cells including adipocytes, preadipocytes, endothelial cells and immune cells such as macrophages (Jung and Choi, 2014). The energy in the adipose tissue is stored in adipocytes in the form of triglycerides. When there is a surplus of triglycerides then adipocytes either increase in numbers (hyperplasia) or increase in size (hypertrophy) (Hausman et al., 2001). Enlargement of adipocytes is related with increased lipolysis, which is associated with T2DM (Engfeldt and Amer, 1988; Weyer et al., 2000). In case of energy need the triglycerides are lipolysed and release free fatty acids (FFAs) into the systemic circulation. FFAs are then transported to the organs and tissues in need of energy. Increased levels of FFAs, in obesity for example, can cause dysfunctions and dysregulations. The FFAs can directly enter the liver and increase lipid synthesis and gluconeogenesis and increase hepatic insulin resistance (Boden, 1997). The FFAs can cause insulin resistance in the peripheral organs (Boden, 1997; Kelley et al., 1993). The FFAs can also trigger cytokine production of immune cells since they are ligands of the Toll-like receptor 4 (TLR-4) (Shi et al., 2006). By activating TLR-4, inflammation is induced which is characterized as a major contributor to obesity-associated metabolic complications (Suganami et al., 2005).

\section{Dyslipidemia}

A relationship between obesity and dyslipidemia with T2DM was observed by Sam and Haffner (Sam et al., 2008). Dyslipidemia is the state at which increased levels of lipids circulate in the blood (Clemente-Postigo et al., 2011). Obesity related dyslipidemia includes high levels of plasma FFAs, low levels of high density lipoprotein (HDL) and abnormal composition of low-density lipoprotein (LDL). This situation occurs when adipose tissue releases increased number of FFAs, through lipolysis. Increased levels of FFAs can decrease the mRNA expression and/or activity of lipoprotein lipase (LPL) (Klop et al., 2013). In addition to the decreased activity of LPL, there is also increased very-low-density-lipoprotein synthesis (VLDL). High levels of VLDL produced in the liver can inhibit the lipolysis of the chylomicrons, leading to hypertriglyceridemia (Jung and Choi, 2014). Consequently, hypertriglyceridemia promotes cholesteryl ester transfer protein-mediated exchange of 


\section{General Introduction}

triglycerides for cholesterol esters between triglyceride-rich lipoproteins such as (VLDL) and lipoproteins, which leads to a decreased HDL-cholesterol concentration and a low number of triglycerides in LDL (Klop et al., 2013). Increased number of triglycerides in LDL are hydrolyzed by hepatic lipase (HL), promoting small dense LDL formation that is associated with higher risk of cardiovascular disease and T2DM (Guendouzi et al., 1999; St Pierre et al., 2005; Mooradian 2008; Rizzo et al., 2009).

\section{Obesity induced inflammation}

As an endocrine organ, the adipose tissue, in the obese state (Figure 2), secrets various chemokines and cytokines, such as monocyte chemotactic protein 1 (MCP-1), tumor necrosis factor alpha (TNF- $\alpha$ ), interleukin 1 (IL-1), interleukin 6 (IL-6) and interleukin 8 (IL-8) that promote insulin resistance (Hotamisligil, et al., 1993; Jung and Choi, 2014; Amrani et al., 1996; Sartipy and Loskutoff 2003; Rotter et al., 2003). Additionally, the macrophages of the adipose tissue change phenotypically and switch from an anti-inflammatory M2 polarization state to a pro-inflammatory M1 polarization state (Lumeng et al., 2007; Jung and Choi, 2014). Consequently, secretion of pro-inflammatory chemokines and cytokines takes place, which is linked to insulin resistance (Jiao et al., 2009). Conclusively, a decreased number of macrophages that infiltrate adipose tissue is correlated with insulin sensitivity (Jung and Choi, 2014). 


\section{Chapter 1}

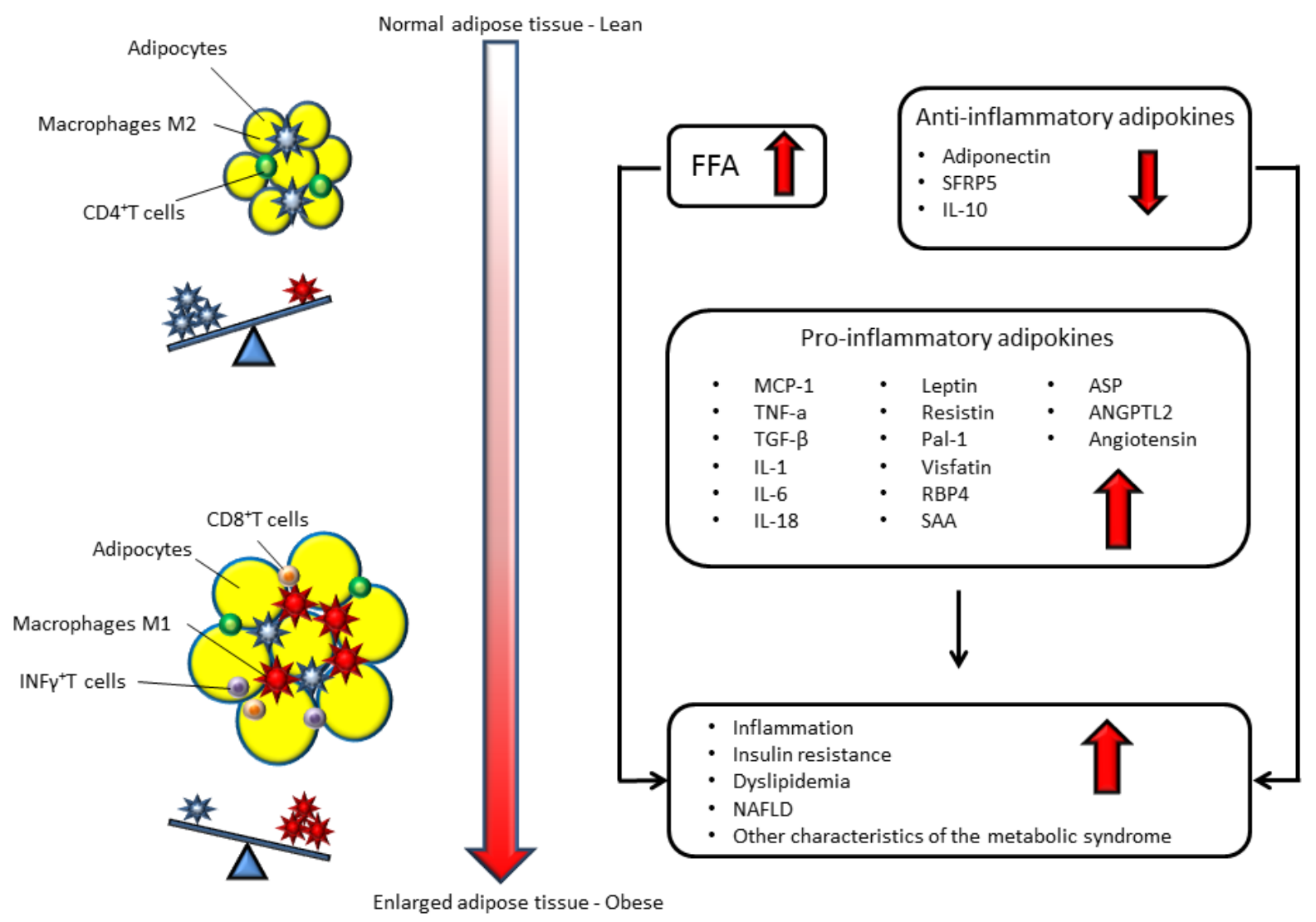

Figure 2. Adipose tissue in normal and obese subjects. In obese state there is an increase in the size of adipocytes and increase of FFAs and pro-inflammatory adipokine secretion. As a consequence there are modifications in inflammatory, glucose and lipid responses. Conversely the levels of anti-inflammatory adipokines are decreased. Additionally a switch in the macrophages phenotype is forced from M2 polarization to M1 polarization. Based on Jung and Choi (2014)

\section{Toll-like receptor 4 (TLR-4)}

Toll-like receptor 4 (TLR-4) was the first characterized mammalian Toll receptor of the Tolllike receptor family, which plays a significant role in the innate immune system acting against microbial pathogens and inducing adaptive immune responses (Medzhitov, 1997; Kawai and Akira 2006; Medzhitov, 2001). It has been reported that TLR-4 detects lipopolysaccharide (LPS) as the ligand and upon ligand binding acts as a signal-transducing receptor (Poltorak et al., 1998, Qureshi et al., 1999; Hoshino et al., 1999; Baccala et al., 2007; Tarkowski et al., 2010). After recognition of LPS, a cascade of signals starts in two pathways (Figure 3). The first is the Myeloid Differentiation primary response gene 88 pathway (MyD88) at which TLR-4 binds to the MyD88 at the TIR (Toll/Interleukin-1 receptor), the cytoplasmic domain of TLR-4. Then the amino-terminal of MyD88 interacts with the amino-terminal of IRAK (IL-1R-associated kinase). Next the formed MyD88-IRAK interacts with TOLLIP (Toll- 


\section{General Introduction}

interacting protein). IRAK is autophosphorylated and it is able to connect with TRAF-6 (TNF-receptor associated factor 6) (Doyle and O’Neil 2006; Medazhitov, 2001; Zangh and Ghosh, 2001). This activates Tat-associated kinase (TAK) and Mitogen activated protein kinase kinase (MKK6) which in turn phosphorylates the IkB kinase (IKK) complex. Subsequently NF-kB and c-Jun N-terminal kinases (JNK) are finally activated (Baccala et al., 2007; Kim 2006; Kawai and Akira, 2006; Medzhitov, 2001; Shi et al., 2006; Tarkowski et al., 2009; Tschopp et al., 2003). Parallel to this pathway TLR-4 has another pathway, the MyD88independent pathway that is based in the connection of TLR-4 to the TIR domain of TIRAP (domain-contain adapter protein) (Kawai and Akira, 2006; Medzhiton, 2001; Mogensen, 2009). TIRAP signals through RNA-dependent protein kinase (PKR) and interferon regulatory factor 3 (Cohen 2002) which later activate NF-kB. NF-kB activation is linked with insulin resistance, T2DM and inflammation (Arkan et al., 2005). Tarkowski and his colleagues showed (2010) that resistin, a cytokine, is competing with LPS for TLR-4 binding.

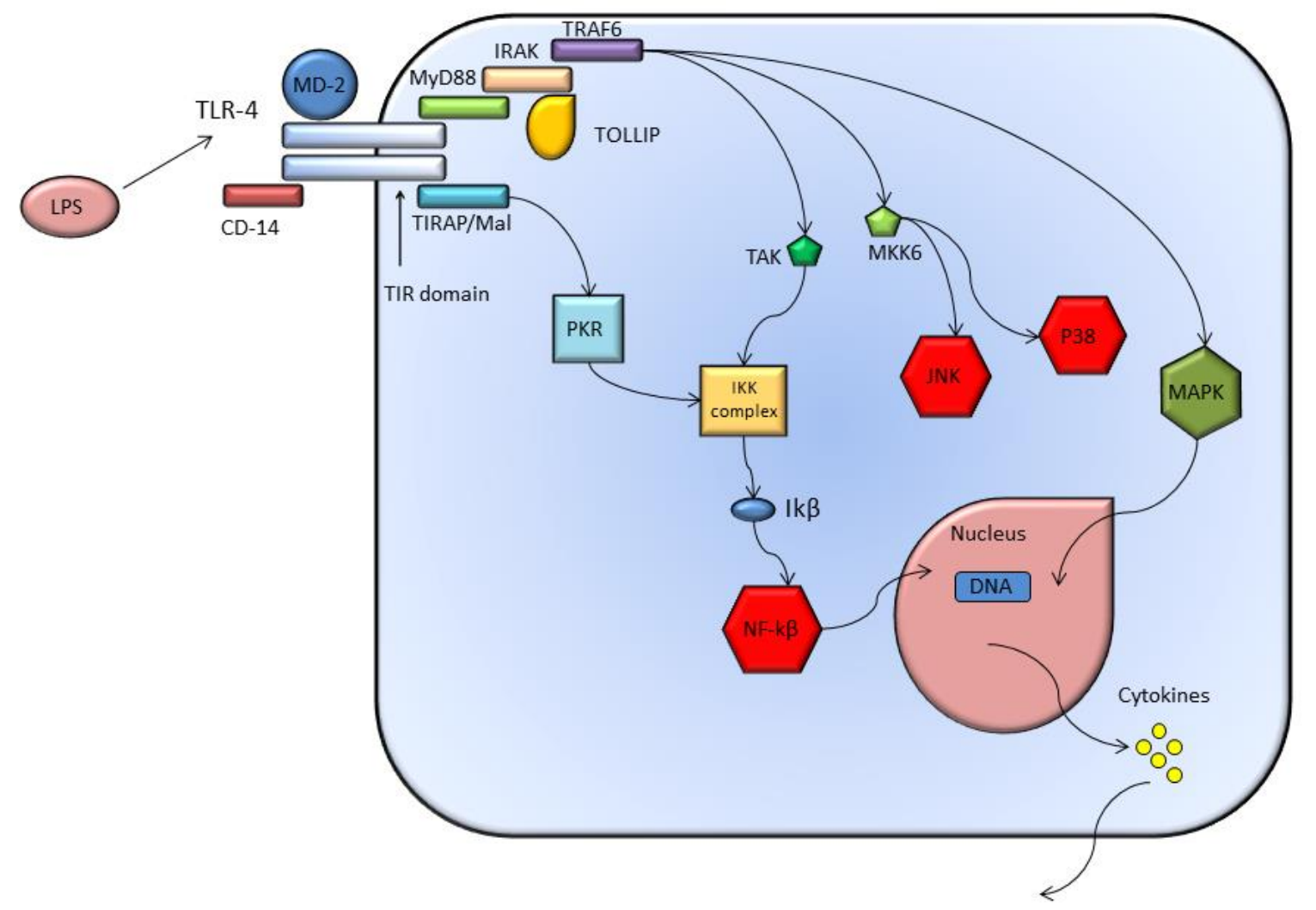

Figure 3. Schematic representation of TLR-4 pathways. The MyD88 pathway follows the activation of IRAK, TRAF and IKK. The MyD88 independent pathway follows the activation of PKR and/or IRF3. Both pathways lead to the activation of NF-kB. Based on Cohen (2002) 


\section{Chapter 1}

\section{Resistin}

Resistin is a $12.5 \mathrm{kDa}$ polypeptide, which belongs to the cysteine-rich proteins termed resistin like molecules (RELMs) (Patel D et al., 2004). In rodents it is secreted by white adipose tissue (McTernan et al., 2002) and in humans basically by adipocytes and macrophages (Patel L et al., 2003). In hyperglycemic and hyperinsulinemic rodents and in humans with T2DM it has been reported that resistin appears in increased levels in plasma (Hartman et al., 2002; Lazar, 2007; Rajala et al., 2004; Stofkova, 2010). The actual receptor of resistin is still unknown. However, several possible pre-diabetic effects of resistin have been reported (Stofkova, 2010). First, resistin could inhibit the intrinsic activity of cell surface glucose transporters as for example the insulin-stimulated 2-deoxyglucose which contributes to glucose uptake (Moon et al., 2003). Second, resistin could suppress GLUT4 translocation (Palanivel et al., 2006) or its gene expression resulting the reduction of insulin capability for glucose uptake (Fu et al., 2006). Third, resistin could promote SOCS-3 expression, which is a known insulin resistance promotor, thus inhibiting insulin signaling (Steppan et al., 2005). Fourth, resistin could block insulin transduction pathways such as PI-3k/Akt (Sheng et al., 2008) as it is shown in figure 1. Fifth, resistin could promote the expression of gluconeogenic enzymes and hinder AMPK activity, correspondingly stimulating the hepatic glucose production (Banerjee et al., 2004). Finally, resistin could activate the release of FFAs from adipose tissue (Pravenec et al., 2006).

\section{$\operatorname{PPAR} \gamma-$ TZDs}

Until recently medication for T2DM was focused on the use of peroxisome proliferatoractivated receptor gamma (PPAR $\gamma)$ agonists. Specific ligands for PPAR $\gamma$ are thiazolidinediones (TZDs) (Berger et al., 2002; Yki-Jarvinen, 2004). However, only pioglitazone has remained in use after troglitazone and rosiglitazone were withdrawn from the market because of hepatotoxicity and increased risk of heart attacks, respectively. In some countries even pioglitazone has been withdrawn from the market because of its possible association with increased incidence of bladder tumors. PPAR $\gamma$ (Figure 4) is a receptor that regulates adipogenesis and glucose homeostasis (Tontonoz and Spiegelman, 2008). As mentioned before dysregulation of these two functions can cause insulin resistance. PPAR $\gamma$ has the ability to promote lipid uptake and storage by the adipose tissue. Uptake of lipids by the peripheral organs would promote insulin resistance (Barnes and Miner, 2009; Boden et al., 2005). Additionally, activation of PPAR $\gamma$ by TZDs in rodents and humans increased the 


\section{General Introduction}

insulin-stimulated glucose uptake thereby counteracting the effects in T2DM (Yki-Jarvinen, 2004). Furthermore, in vitro research in mice has shown that PPAR $\gamma$ activation by various TZDs is correlated with the minimum effective dose (MED) for their antihyperglycemic activity (Willson et al., 1996). The insulin sensitizing effects of TZDs could be related to the binding and activation of the PPAR $\gamma$ receptor. In addition, PPAR $\gamma$ activation has been correlated to the downregulation of adipokines, such as resistin which promote inflammation and eventually lead to insulin resistance.

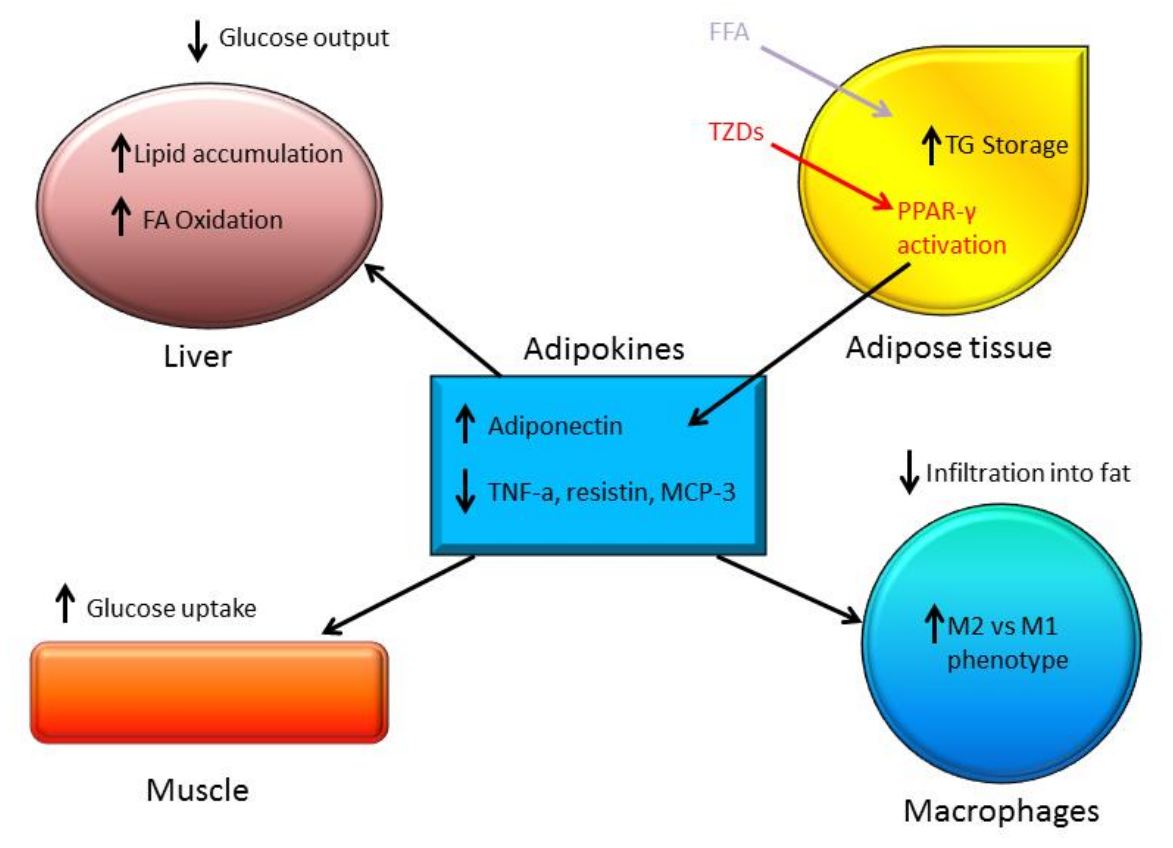

Figure 4. PPAR $\gamma$ activation by TZDs modulates glucose and lipid metabolism, leading to improved insulin sensitivity. Activation of PPAR $\gamma$ increases lipid uptake and storage. Activation of PPAR $\gamma$ also increases the production of anti-inflammatory adipokines, such as adiponectin, and decreases the production pro-inflammatory adipokines such as resistin, TNF-alpha and MCP-1. Additionally PPAR $\gamma$ forces macrophages towards antiinflammatory M2 phenotype, reducing the macrophage infiltration into fat. Based on Tontonoz and Spiegelman (2008)

\section{Other possible targets for drug development}

More recently due to the side effects resulting in the withdrawal of the majority of the TZDs from the market, research has been redirected away from PPAR $\gamma$ and the TZDs focusing on other possible targets for drug development. These include in addition to resistin, already described above and the major focus of the present thesis, incretin hormone glucagon-like peptide 1 receptor (GLP-1R) agonists, dipeptidyl peptidase-4 (DPP-4) inhibitors and sodiumglucose cotransporter type 2 (SGLT-2) inhibitors. The first two are focusing on targeting the "incretin effect", which is the glucose-dependent increase of insulin stimulation in response to nutrients, in comparison with the glucose infused intravenously, and is decreased in T2DM 


\section{Chapter 1}

(Holst and Orskov 2004; Nauck 2016; Lutz and Osto 2016). More specifically GLP-1 is a gut hormone and has been found to stimulate insulin secretion. DPP-4 is responsible for the degradation and inactivation of incretin hormones. Therefore finding GLP-1 agonists and/or DPP-4 inhibitors might be of use in finding targets for drug development for T2DM (Graaf et al. 2016). Examples of GLP-1R agonists are exenatide, liraglutide, lixisenatide, dulaglutide and albiglutide (Nauck 2016). Examples of DPP-4 inhibitors are sigagliptin, saxagliptin, linagliptin and alogliptin (Nauck 2016). On the other hand inhibition of SGLT-2, which is involved in glucose reabsorption in the blood after it is filtered by the kidney, uses a more hemodynamic approach, since it is targeting the kidney so as to increase urinary glucose excretion (Reed 2016; Scheen 2016). Examples of SGLT-2 inhibitors are canagliflozin, dapaglifloxin and empagliflozin.

\section{Outline of the thesis}

Type 2 Diabetes Mellitus (T2DM) is a modern metabolic disorderand results when the body is unable to produce sufficient amount of insulin or does not respond properly to insulin, the hormone that regulates the level of glucose in blood (Saltiel et al., 2001). The increase of glucose in the blood may cause hyperglycemia that leads to long term complications. On the other hand, a decrease of glucose levels may cause hypoglycemia that might lead to coma and ultimately to death (Guo et al., 2009).

T2DM is the most common type and is a result of impaired activity of $\beta$-cells, which are the cells that produce insulin, in combination with the insulin resistance of the insulin-targeted organs. It is closely related with the metabolic syndrome which is described by abdominal obesity, atherogenic dyslipidemia, elevated blood pressure, insulin resistance and prothrombotic and proinflammatory states (Tenenbaum et al., 2003). Until now, there are no easy medical treatments for diabetes. Additionally, the treatments do not have a preventive character for the complications associated with diabetes, such as neuropathy, retinopathy and nephropathy (Segev et al., 2003). Usually medical treatments focus on the use of exogenous insulin to control the level of glucose or on the transplantation of the whole-pancreas organ which has been the most effective treatment for diabetic people with severe complications, until now (Bonner-Weir et al., 2005). One of the major drawbacks is the limited supply of donor tissues (Weir et al., 1997).

The aim of the present thesis was to define novel targets for the possible development of drugs for T2DM. Optimization of present preventive therapies including development of less 


\section{General Introduction}

expensive drugs may be of help. Such new drugs may be analogues to presently prescribed drugs like for example pioglitazone and rosiglitazone which belong to the thiazolidinedione class of insulin sensitizing compounds that act as PPAR $\gamma$ agonists, and have an important role in lipid metabolism and insulin sensitization. The structure activity relationship between PPAR $\gamma$ agonists and their glucose lowering activity has been well established (Willson et al., 1996). However given the serious side effects there is a need for definition of other targets for development of drugs for T2DM. Amongst possible novel targets, adipokines such as resistin that promote insulin resistance have been identified as possible novel targets for development of drugs for T2DM, since resistin has been reported to be detected at higher levels in plasma of T2DM patients (Owecki et al., 2011) and has also been reported to be downregulated upon PPAR $\gamma$ activation (Saltiel et al., 2001). Thus, the aim of the present thesis was to define novel targets for the possible development of drugs for T2DM with emphasis on resistin related mechanisms.

Chapter one of the thesis gives an introduction and a global overview of T2DM, insulin function and the adipose tissue as well as of the modes of action underlying the adverse effects in T2DM. T2DM results from impaired insulin production of the $\beta$-cells of the pancreas and from the inability of the peripheral organs targeted by insulin to exert adequate glucose uptake from the blood, leading to insulin resistance. In this thesis we focused especially on resistin as a possible new drug strategy and novel drug target that deals with insulin resistance.

Given that PPAR $\gamma$ activation is an important target for TZDs, drugs that were withdrawn for too serious side effects but were effective against T2DM, in chapter 2 we investigated whether for a series of related TZDs the level of activation of PPAR $\gamma$ detected by a CALUX reporter gene assay would correlate with their potential for resistin downregulation in mouse adipocytes, and whether the $\mathrm{EC}_{50}$ s for resistin downregulation in the mouse adipocytes would correlate with the MED reported for their antihyperglycemic activity in a mouse model (Willson et al., 1996). If such correlations would be established it would support the use of resistin downregulation as an additional and possibly more direct target for the development of antihyperglycemic compounds.

Given that resistin may be an important new target for development of drugs for T2DM and that the TLR-4 receptor may be an important target for resistin mediated induction of biological effects, in chapter 3 we investigated the possible use of a TLR-4 signaling reporter 


\section{Chapter 1}

gene cell line to detect antagonists for resistin mediated effects. Studies have shown that resistin competes with LPS for TLR-4 binding (Tarkowsky, 2010). Consequently, the inhibition of resistin induced TLR-4 signaling could provide a new mechanism and screening tool to detect and develop new T2DM drugs able to counteract resistin induced insulin resistance.

Resistin is proven to promote insulin resistance with a possible intermediate role for the TLR4 receptor although the actual receptor(s) of resistin and the actual pathway(s) that resistin uses are still unknown. In chapter 4 we investigated the effects of resistin in human adipocytes. We used an RNA sequencing method to obtain detailed insight in the effects of resistin in adipocytes and possibly identify novel biomarkers that could be useful for the development of new T2DM medicines.

In chapter 5 we used a novel in vitro model for T2DM, combining adipocytes with activated macrophages. This assay was used to screen for new proteins that could be involved in T2DM. The aim was to identify novel protein markers that can be of interests for research into the mechanism of T2DM development and additional risk assessment of obese patients prior to the onset of T2DM. In addition, our data were compared to in vivo data sets from mice and humans to further investigate whether some of these proteins have the potential to be used as diagnostic tools/biomarkers which would allow for a better and more personalized treatment of T2DM patients.

Finally in chapter 6 a general discussion is presented with remarks about the findings in this thesis and some future perspectives about the methods used but also for the general problem of T2DM.

\section{References}

Ahima, R. S. and J. S. Flier (2000). "Adipose tissue as an endocrine organ." Trends Endocrinol Metab 11(8): 327-332.

Amrani, A., et al. (1996). "Interleukin-1 effect on glycemia in the non-obese diabetic mouse at the pre-diabetic stage." J Endocrinol 148(1): 139-148.

Arkan, M. C., et al. (2005). "IKK-beta links inflammation to obesity-induced insulin resistance." Nat Med 11(2): 191-198.

Baccala, R., et al. (2007). "TLR-dependent and TLR-independent pathways of type I interferon induction in systemic autoimmunity." Nat Med 13(5): 543-551. 


\section{General Introduction}

Banerjee, R. R., et al. (2004). "Regulation of fasted blood glucose by resistin." Science 303(5661): 1195-1198.

Barnes, K. M. and J. L. Miner (2009). "Role of resistin in insulin sensitivity in rodents and humans." Curr Protein Pept Sci 10(1): 96-107.

Berger, J. P., et al. (2005). "PPARs: therapeutic targets for metabolic disease." Trends Pharmacol Sci 26(5): 244-251.

Boden, G. (1997). "Role of fatty acids in the pathogenesis of insulin resistance and NIDDM." Diabetes 46(1): 3-10.

Boden, G. (2011). "Obesity, insulin resistance and free fatty acids." Curr Opin Endocrinol Diabetes Obes 18(2): 139-143.

Boden, G., et al. (2005). "Thiazolidinediones upregulate fatty acid uptake and oxidation in adipose tissue of diabetic patients." Diabetes 54(3): 880-885.

Bonner-Weir, S. and G. C. Weir (2005). "New sources of pancreatic beta-cells." Nat Biotechnol 23(7): 857-861.

Chen, L., et al. (2012). "The worldwide epidemiology of type 2 diabetes mellitus--present and future perspectives." Nat Rev Endocrinol 8(4): 228-236.

Clemente-Postigo, M., et al. (2011). "Adipose tissue gene expression of factors related to lipid processing in obesity." PLoS One 6(9): e24783.

Cohen, J. (2002). "The immunopathogenesis of sepsis." Nature 420(6917): 885-891.

Delarue, J. and C. Magnan (2007). "Free fatty acids and insulin resistance." Curr Opin Clin Nutr Metab Care 10(2): 142-148.

Doyle, S. L., et al. (2007). "Signaling by Toll-like receptors 8 and 9 requires Bruton's tyrosine kinase." J Biol Chem 282(51): 36953-36960.

Engfeldt, P. and P. Arner (1988). "Lipolysis in human adipocytes, effects of cell size, age and of regional differences." Horm Metab Res Suppl 19: 26-29.

Ferrannini, E. (1992). "The insulin resistance syndrome." Curr Opin Nephrol Hypertens 1(2): 291-298.

$\mathrm{Fu}$, Y., et al. (2006). "Proinflammatory cytokine production and insulin sensitivity regulated by overexpression of resistin in 3T3-L1 adipocytes." Nutr Metab (Lond) 3: 28.

Galic, S., et al. (2010). "Adipose tissue as an endocrine organ." Mol Cell Endocrinol 316(2): 129-139.

Graaf de, C., (2016) "Glucagon-Like Peptide-1 and Its Class B G Protein-Coupled Receptors: A Long March to Therapeutic Successes”. Pharmacol Rev 68(4): 954-1013.

Guendouzi, K., et al. (1999). "Biochemical and physical properties of remnant-HDL2 and of pre beta 1-HDL produced by hepatic lipase." Biochemistry 38(9): 2762-2768.

Guo, T. and M. Hebrok (2009). "Stem cells to pancreatic beta-cells: new sources for diabetes cell therapy." Endocr Rev 30(3): 214-227. 


\section{Chapter 1}

Hartman, H. B., et al. (2002). "Mechanisms regulating adipocyte expression of resistin." J Biol Chem 277(22): 19754-19761.

Hausman, D. B., et al. (2001). "The biology of white adipocyte proliferation." Obes Rev 2(4): 239-254.

Holst, J.J., Orskov, C. (2004) "The incretin approach for diabetes treatment: modulation of islet hormone release by GLP-1 agonism.” Diabetes. 53 Suppl 3:S197-204.

Hoshino, K., et al. (1999). "Cutting edge: Toll-like receptor 4 (TLR4)-deficient mice are hyporesponsive to lipopolysaccharide: evidence for TLR4 as the Lps gene product." J Immunol 162(7): 3749-3752.

Hotamisligil, G. S., et al. (1993). "Adipose expression of tumor necrosis factor-alpha: direct role in obesity-linked insulin resistance." Science 259(5091): 87-91.

Jiao, P., et al. (2009). "Obesity-related upregulation of monocyte chemotactic factors in adipocytes: involvement of nuclear factor-kappaB and c-Jun NH2-terminal kinase pathways." Diabetes 58(1): 104-115.

Jung, U. J. and M. S. Choi (2014). "Obesity and its metabolic complications: the role of adipokines and the relationship between obesity, inflammation, insulin resistance, dyslipidemia and nonalcoholic fatty liver disease." Int J Mol Sci 15(4): 6184-6223.

Kahn, B. B. and J. S. Flier (2000). "Obesity and insulin resistance." J Clin Invest 106(4): 473481.

Kawai, T. and S. Akira (2006). "TLR signaling." Cell Death Differ 13(5): 816-825.

Kelley, D. E., et al. (1993). "Interaction between glucose and free fatty acid metabolism in human skeletal muscle." J Clin Invest 92(1): 91-98.

Kershaw, E. E. and J. S. Flier (2004). "Adipose tissue as an endocrine organ." J Clin Endocrinol Metab 89(6): 2548-2556.

Kim, J. K. (2006). "Fat uses a TOLL-road to connect inflammation and diabetes." Cell Metab 4(6): 417-419.

Klop, B., et al. (2013). "Dyslipidemia in obesity: mechanisms and potential targets." Nutrients 5(4): 1218-1240.

Lazar, M. A. (2007). "Resistin- and Obesity-associated metabolic diseases." Horm Metab Res 39(10): 710-716.

Lumeng, C. N., et al. (2007). "Obesity induces a phenotypic switch in adipose tissue macrophage polarization." J Clin Invest 117(1): 175-184.

Lutz, T.A., Osto, E.(2016) "Glucagon-like peptide-1, glucagon-like peptide-2, and lipid metabolism." Curr Opin Lipidol 27(3):257-63. doi: 10.1097/MOL.0000000000000293.

McTernan, C. L., et al. (2002). "Resistin, central obesity, and type 2 diabetes." Lancet 359(9300): 46-47. 


\section{General Introduction}

McTernan, P. G., et al. (2002). "Increased resistin gene and protein expression in human abdominal adipose tissue." J Clin Endocrinol Metab 87(5): 2407.

Medzhitov, R. (2001). "Toll-like receptors and innate immunity." Nat Rev Immunol 1(2): 135-145.

Mogensen, T. H. (2009). "Pathogen recognition and inflammatory signaling in innate immune defenses." Clin Microbiol Rev 22(2): 240-273, Table of Contents.

Moon, B., et al. (2003). "Resistin inhibits glucose uptake in L6 cells independently of changes in insulin signaling and GLUT4 translocation." Am J Physiol Endocrinol Metab 285(1): E106-115.

Mooradian, A. D. (2009). "Dyslipidemia in type 2 diabetes mellitus." Nat Clin Pract Endocrinol Metab 5(3): 150-159.

Nauck, M. (2016). Incretin therapies : highlighting common features and differences in the modes of action of glucagon-like peptide- 1 receptor agonists and dipeptidyl peptidase- 4 inhibitors, 203-216.

Olokoba, A. B., et al. (2012). "Type 2 diabetes mellitus: a review of current trends." Oman Med J 27(4): 269-273.

Owecki, M., et al. (2011). "Serum resistin concentrations are higher in human obesity but independent from insulin resistance." Exp Clin Endocrinol Diabetes 119(2): 117-121.

Palanivel, R., et al. (2006). "Regulation of insulin signaling, glucose uptake and metabolism in rat skeletal muscle cells upon prolonged exposure to resistin." Diabetologia 49(1): 183190.

Patel, L., et al. (2003). "Resistin is expressed in human macrophages and directly regulated by PPAR gamma activators." Biochem Biophys Res Commun 300(2): 472-476.

Patel, S. D., et al. (2004). "Disulfide-dependent multimeric assembly of resistin family hormones." Science 304(5674): 1154-1158.

Petersen, K. F. and G. I. Shulman (2006). "Etiology of insulin resistance." Am J Med 119(5 Suppl 1): S10-16.

Poltorak, A., et al. (1998). "Genetic and physical mapping of the Lps locus: identification of the toll-4 receptor as a candidate gene in the critical region." Blood Cells Mol Dis 24(3): 340-355.

Pravenec, M., et al. (2006). "Fat-specific transgenic expression of resistin in the spontaneously hypertensive rat impairs fatty acid re-esterification." Int J Obes (Lond) 30(7): 1157-1159.

Qureshi, S. T., et al. (1999). "The Lps locus: genetic regulation of host responses to bacterial lipopolysaccharide." Inflamm Res 48(12): 613-620.

Rajala, M. W., et al. (2004). "Regulation of resistin expression and circulating levels in obesity, diabetes, and fasting." Diabetes 53(7): 1671-1679. 


\section{Chapter 1}

Reed J.W., (2016).'Impact of sodium-glucose cotransporter 2 inhibitors on blood pressure." Vasc Health Risk Manag. 27;12:393-405.

Rizzo, M., et al. (2009). "Small, dense low-density lipoproteins (LDL) are predictors of cardio- and cerebro-vascular events in subjects with the metabolic syndrome." Clin Endocrinol (Oxf) 70(6): 870-875.

Rotter, V., et al. (2003). "Interleukin-6 (IL-6) induces insulin resistance in 3T3-L1 adipocytes and is, like IL-8 and tumor necrosis factor-alpha, overexpressed in human fat cells from insulin-resistant subjects." J Biol Chem 278(46): 45777-45784.

Saltiel, A. R. and C. R. Kahn (2001). "Insulin signalling and the regulation of glucose and lipid metabolism." Nature 414(6865): 799-806.

Sam, S., et al. (2008). "Relationship of abdominal visceral and subcutaneous adipose tissue with lipoprotein particle number and size in type 2 diabetes." Diabetes 57(8): 2022-2027.

Sartipy, P. and D. J. Loskutoff (2003). "Monocyte chemoattractant protein 1 in obesity and insulin resistance." Proc Natl Acad Sci U S A 100(12): 7265-7270.

Scheen A,J., (2016). "DPP-4 inhibitor plus SGLT-2 inhibitor as combination therapy for type 2 diabetes: from rationale to clinical aspects.” Expert Opin Drug Metab Toxicol. 29:1-11.

Schinner, S., et al. (2005). "Protein kinase B activity is sufficient to mimic the effect of insulin on glucagon gene transcription." J Biol Chem 280(8): 7369-7376.

Schinner, S., et al. (2005). "Molecular mechanisms of insulin resistance." Diabet Med 22(6): 674-682.

Segev, H., et al. (2004). "Differentiation of human embryonic stem cells into insulinproducing clusters." Stem Cells 22(3): 265-274.

Sheng, C. H., et al. (2008). "Resistin is expressed in human hepatocytes and induces insulin resistance." Endocrine 33(2): 135-143.

Shi, H., et al. (2006). "TLR4 links innate immunity and fatty acid-induced insulin resistance." J Clin Invest 116(11): 3015-3025.

Steppan, C. M. and M. A. Lazar (2004). "The current biology of resistin." J Intern Med 255(4): 439-447.

Steppan, C. M., et al. (2005). "Activation of SOCS-3 by resistin." Mol Cell Biol 25(4): 15691575.

Stofkova, A. (2010). "Resistin and visfatin: regulators of insulin sensitivity, inflammation and immunity." Endocr Regul 44(1): 25-36.

St-Pierre, A. C., et al. (2005). "Low-density lipoprotein subfractions and the long-term risk of ischemic heart disease in men: 13-year follow-up data from the Quebec Cardiovascular Study." Arterioscler Thromb Vasc Biol 25(3): 553-559.

Suganami, T., et al. (2005). "A paracrine loop between adipocytes and macrophages aggravates inflammatory changes: role of free fatty acids and tumor necrosis factor alpha." Arterioscler Thromb Vasc Biol 25(10): 2062-2068. 


\section{General Introduction}

Tarkowski, A., et al. (2010). "Resistin competes with lipopolysaccharide for binding to tolllike receptor 4." J Cell Mol Med 14(6B): 1419-1431.

Tenenbaum, A., et al. (2003). "Metabolic syndrome and type 2 diabetes mellitus: focus on peroxisome proliferator activated receptors (PPAR)." Cardiovasc Diabetol 2: 4.

Tontonoz, P. and B. M. Spiegelman (2008). "Fat and beyond: the diverse biology of PPARgamma." Annu Rev Biochem 77: 289-312.

Tschopp, J., et al. (2003). "NALPs: a novel protein family involved in inflammation." Nat Rev Mol Cell Biol 4(2): 95-104.

Wang, Y. C., et al. (2011). "Health and economic burden of the projected obesity trends in the USA and the UK." Lancet 378(9793): 815-825.

Weir, G. C. and S. Bonner-Weir (1997). "Scientific and political impediments to successful islet transplantation." Diabetes 46(8): 1247-1256.

Weyer, C., et al. (2000). "Enlarged subcutaneous abdominal adipocyte size, but not obesity itself, predicts type II diabetes independent of insulin resistance." Diabetologia 43(12): 1498-1506.

Willson, T. M., et al. (1996). "The structure-activity relationship between peroxisome proliferator-activated receptor gamma agonism and the antihyperglycemic activity of thiazolidinediones." Journal of Medicinal Chemistry 39(3): 665-668.

Yki-Jarvinen, H. (2004). "Thiazolidinediones." N Engl J Med 351(11): 1106-1118.

Zhang, G. and S. Ghosh (2001). "Toll-like receptor-mediated NF-kappaB activation: a phylogenetically conserved paradigm in innate immunity." J Clin Invest 107(1): 13-19. 



\section{Chapter 2}

\section{Correlation between activation of PPAR $\gamma$} and resistin downregulation in a mouse

\section{adipocyte cell line by a series of thiazolidinediones.}
A. Sotiriou
R. H. Blaauw
C. Meijer
L. H. Gijsbers
B. van der Burg
J. Vervoort

Ivonne M.C.M. Rietjens 


\title{
Chapter 2
}

\begin{abstract}
The present study shows significant correlations between the $\mathrm{EC}_{50}$ for PPAR $\gamma$ activation in a reporter gene cell line and resistin downregulation in mouse adipocytes, and between the $\mathrm{IC}_{50}$ for resistin downregulation and the already published minimum effective dose for antihyperglycemic activity in a mouse model. These correlations indicate that PPAR $\gamma$ mediated downregulation of resistin might promote insulin sensitivity and that downregulation of resistin in mouse adipocytes provides an adequate and possibly more direct bioassay for screening of newly developed antihyperglycemic compounds. Because of the higher throughput of the PPAR $\gamma$ the resistin downregulation assays seems most suitable to be used as a second tier in a tiered screening strategy.
\end{abstract}




\section{Introduction}

Insulin resistance is the state at which the insulin targeted organs are not responding to insulin signaling (Lazar, 2007). It is a characteristic feature of human and rodent obesity and type 2 diabetes mellitus (Ferrannini, 1992). The mechanism underlying insulin resistance is poorly understood, but the phenomenon is strongly associated with increased adipogenesis and increased serum levels of free fatty acids (Boden, 1997; Lazar, 2007; Reitman et al., 2000). Excessive deposition of fat not only in adipocytes but also in peripheral organs, such as skeletal muscles and liver, can lead to insulin resistance (Tarcin et al., 2007). Consequently medication that reduces or eliminates insulin resistance is needed for the prevention or the treatment of type 2 diabetes mellitus.

Current drug development strategies for type 2 diabetes focus on the development of peroxisome proliferator-activated receptor gamma (PPAR $\gamma$ ) agonists. Thiazolidinediones (TZDs) are an example of such insulin sensitizing compounds that are ligand specific to PPAR $\gamma$ (Berger and Moller, 2002; Yki-Jarvinen, 2004). Currently only pioglitazone is used as medication, after the withdrawal of troglitazone in 2004 and rosiglitazone in 2011 from the market because of suspected hepatotoxicity and increased risk of heart attacks, respectively.

Studies have shown that the effect the TZDs have on insulin resistance is related to the activation of PPAR $\gamma$ in their primary target tissue being the adipose tissue (Lehrke and Lazar, 2005). PPAR $\gamma$ is a receptor that regulates adipogenesis and glucose homeostasis (Tontonoz and Spiegelman, 2008). Several studies have shown that poor functioning of adipogenesis and glucose homeostasis can lead to insulin resistance. Activation of PPAR $\gamma$ promotes the uptake of lipids in the adipose tissue and not in peripheral organs such as the liver and muscle. Uptake of the lipids in peripheral organs reflects the insulin resistant state (Barnes and Miner, 2009; Boden et al., 2005). Additionally activation of PPAR $\gamma$ is correlated with the production of smaller more insulin-sensitive adipocytes (Okuno et al., 1998). Treatment with the TZDs rosiglitazone, pioglitazone and troglitazone increased the insulin-stimulated glucose uptake in peripheral organs in rodents and in human (Yki-Jarvinen, 2004). Willson and his colleagues (Willson et al., 1996) have shown that for a series of structurally related TZDs their $\mathrm{EC}_{50}$ for PPAR $\gamma$ activation detected in an in vitro reporter gene assay could be correlated to the minimum effective dose (MED) for their antihyperglycemic activity characterised in an in vivo mouse model. 


\section{Chapter 2}

Thus, the biological effects of the TZDs on insulin sensitivity have been ascribed to their binding to and activation of PPAR $\gamma$ that might be involved in the insulin sensitizing effects of TZDs (Tontonoz and Spiegelman, 2008). A possible central downstream mechanism for the insulin sensitizing effects of TZDs is the modulation of bioactive molecules such as adipokines (Tontonoz and Spiegelman, 2008). Activation of PPAR $\gamma$, resulting downregulation of the adipokine resistin, has been suggested to be involved in the mechanism underlying the insulin sensitising effect of TZDs (Tontonoz and Spiegelman, 2008).

Resistin is a $12.5 \mathrm{kDa}$ polypeptide, belonging to the family of cysteine-rich proteins termed RELMs (Resistin-Like Molecules) (Patel D. et al., 2004). It is secreted by the white adipose tissue in mice (McTernan P. et al., 2002) and by adipose tissue and macrophages in humans (Patel L et al., 2003). Increased levels of resistin in hyperglycemic and hyperinsulinemic rodents and in obese and type 2 diabetes mellitus human have been reported (Hartman et al., 2002; Lazar, 2007; Rajala et al., 2004; Stofkova, 2010). Upon PPAR $\gamma$ activation by the TZD rosiglitazone, resistin was reported to be downregulated (Saltiel and Kahn, 2001; Steppan et al., 2001). It has been suggested that resistin is involved in insulin resistance by interfering with the insulin signaling pathway, however its receptor causing the downstream effects on insulin sensitivity remains to be established (Barnes and Miner, 2009; Daquinag et al., 2011; Haugen et al., 2001; McTernan P. et al., 2003; Sanchez-Solana et al., 2012; Tarkowski et al., 2010). The aim of the present study was to investigate whether for a series of related TZDs the level of activation of PPAR $\gamma$ detected by a recently developed reporter gene assay would correlate with their potential for resistin downregulation in mouse adipocytes, and whether the $\mathrm{EC}_{50} \mathrm{~s}$ for resistin downregulation in the mouse adipocytes would correlate with the MED reported for their antihyperglycemic activity in a mouse model (Willson et al., 1996). If such correlations would be established it would support the use of resistin downregulation as an additional and possibly more direct target for the development of antihyperglycemic compounds.

\section{Materials and methods}

\section{Thiazolidinediones}

The structures of the TZDs that were used in this study are shown in Figure 1. Rosiglitazone (CAS Nr: 122320-73-4), CAY-10415 (CAS Nr: 146062-49-9), CAY-10506 (CAS Nr: 292615-75-9), ciglitazone (CAS no. 74772-77-3), pioglitazone (CAS no. 111025-46-8) were purchased from Cayman chemicals (Ann Arbor, Michigan). DRF-2519 (CAS Nr: 194713-46- 
7) was purchased from Sigma (St. Louis, USA). 5,4-Fluorobenzilidene-2,4-TZD (CAS Nr: 262601-87-6) was purchased from Santa Cruz Chemicals (California, USA). Englitazone (CAS Nr: 122228-35-7) was purchased from Dalton Chemicals Services (Toronto, Canada). CS-7017 (CAS Nr : 223132-38-5) (Shimazaki et al., 2008), BRL-48482 (Buckle et al., 1996; Imran et al., 2007), DRF-2189 (CAS Nr : 172647-53-9) (Lohray et al., 1998), (Z)-5-[[4-[2-(2Benzoxazolylmethylamino) ethoxy]phenyl]-methylene]-2,4-thiazolidinedione (Cantello et al., 1994) (from now on called 65 as in the reference), (Z)-5-[[4-[2-(Methyl-2pyridinylamino)ethoxy]phenyl]-methylene]-2,4-thiazolidinedione (Cantello et al., 1994) (from now on called 66 as in the reference) and PMT-13 (Chakrabarti et al., 2002; Imran et al., 2007) were synthesized by Chiralix (Nijmegen, Netherlands) according to literature (Buckle et al.,1996; Cantello et al., 1994; Chakrabarti et al., 2002; Lohray et al., 1998; McTernan P. et al., 2003) and the purity and identity of the compounds was confirmed by ${ }^{1} \mathrm{H}$ nuclear magnetic resonance spectroscopy ( $\left.{ }^{1} \mathrm{H}-\mathrm{NMR}\right)$ performed on a Varian $400 \mathrm{MHz}$ spectrometer (Palo Alto, California) and compared to data reported in literature (Buckle et al., 1996; Cantello et al., 1994; Chakrabarti et al., 2002; Lohray et al., 1998; McTernan P. et al., 2003). All compounds were tested for cytotoxicity using the MTT test performed as described previously (Hussain et. al., 1993).

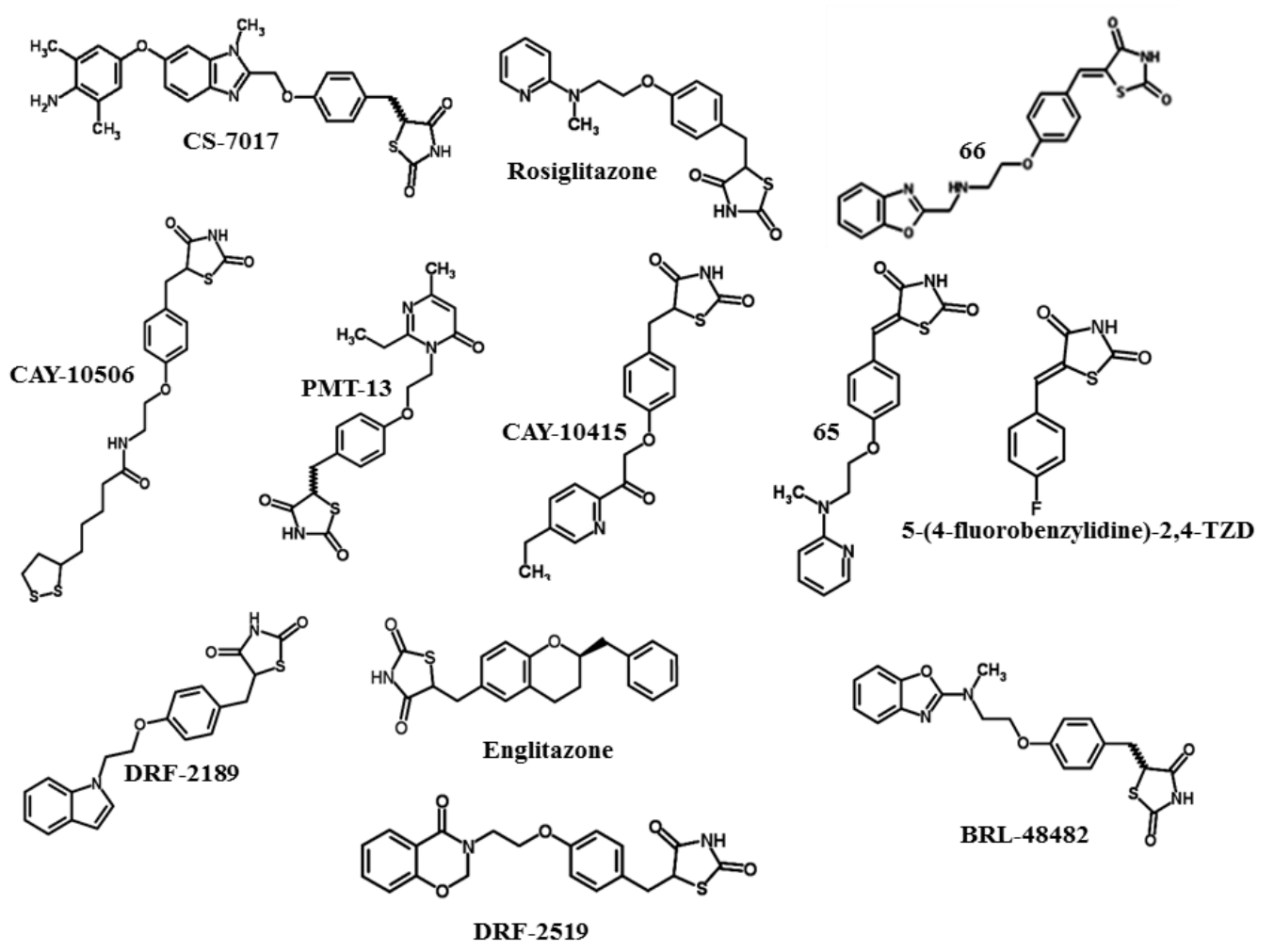

Figure 1. Chemical structure of TZDs tested for PPAR- $\gamma$ activation and resistin downregulation 


\section{Chapter 2}

\section{Reporter gene cell line and assay}

The cell line that was used for this study is a CALUX reporter gene cell line consisting of U2OS cells stably transfected with a 6xPPREtataLuc construct and an expression vector specific for PPAR $\gamma 2$ described in detail elsewhere (Gijsbers et al., 2011). Cells of this PPAR $\gamma 2$ CALUX reporter cell line were cultured in Dulbecco's modified Eagle's medium and Ham's F12 medium (DMEM with high glucose and l-glutamine)(Glutamax, Invitrogen, Breda, Netherlands) supplemented with 7.5\% Fetal Calf Serum (FCS, Invitrogen), 1\% NonEssential Amino acid (NEAA, Invitrogen) and $0.1 \%$ penicillin/streptomycin $(10 \mathrm{u} / \mathrm{ml}-$ $10 \mu \mathrm{g} / \mathrm{ml}$, Invitrogen). Once per week, $200 \mu \mathrm{g} / \mathrm{ml} \mathrm{G} 418$ was added to the culture medium in order to keep selection pressure on the cells.

On the first day cells were trypsinised and diluted in assay medium (DMEM: F12 without phenol red), supplemented with 5\% stripped serum (DCC FCS), 1\% NEAA 100x and 0.1\% penicilium/streptomycin and plated in a 96 well plate at 10000 cells/well. On the second day cells were exposed to TZDs at concentrations of $100 \mathrm{pM}$ up to $10 \mu \mathrm{M}$ for $24 \mathrm{~h}$ with the exception of DRF-2189 that was tested at concentrations of $1 \mathrm{nM}$ up to $10 \mu \mathrm{M}$. On the third day, cells were lysed and luciferase activity was measured as described before (Gijsbers et al., 2011). To this end $100 \mu \mathrm{l}$ of Flash Mix (20 mM tricine, $1.07 \mathrm{mM}\left(\mathrm{MgCO}_{3}\right)_{4} \mathrm{Mg}(\mathrm{OH})_{2} 5 \mathrm{H}_{2} \mathrm{O}$, $2.67 \mathrm{mM} \mathrm{MgSO}_{4}, 0.1 \mathrm{mM}$ EDTA, $2.0 \mathrm{mM}$ dithiothreitol, $470 \mu \mathrm{M}$ D-luciferine, and $5.0 \mathrm{mM}$ ATP) were added per well and light production was measured directly using a luminometer (Anthos, Eugendorf, Austria) and expressed as relative light units (RLUs).

\section{T3-L1 preadipocytes}

3T3-L1 fibroblast-like preadipocytes were cultured in DMEM with high glucose and 1glutamine, supplemented with 10\% Newborn Calf Serum (NCS, Thermo Fisher Scientific Rockford, Illinois) and 1\% penicillin/streptomycin/ampicilin (Sigma, St.Louis, Missouri) and incubated at $37^{\circ} \mathrm{C}$ at $5 \% \mathrm{CO}_{2}$. The cells were split 1:10 twice every week. The confluence during culturing was kept below $80 \%$.

\section{Differentiation to adipocytes}

For the differentiation of pre-adipocytes into adipocytes, 16000 3T3-L1 cells per well were seeded in 6-well plates and incubated until confluence. After 2 days of confluence the cells were ready for differentiation. The medium was changed to MDI (methylxanthine dexamethasone - insulin) induction medium being DMEM with high glucose and l-glutamine 
supplemented with $10 \%$ FCS, $0.5 \mathrm{mM}$ 3-isobutyl-1-methylxanthine (IBMX), $0.5 \mu \mathrm{g} / \mathrm{ml}$ insulin, $0.1 \mu \mathrm{M}$ dexamethasone on day 0 and incubated for 3 days. After three days, the MDI induction medium was changed to insulin medium (Insulin medium being DMEM high glucose and l-glutamine supplemented with $10 \%$ FCS, $0.5 \mu \mathrm{g} / \mathrm{ml}$ insulin) and incubated for 3 days. On day 6, the medium was changed to DMEM high glucose and l-glutamine supplemented with $10 \%$ FCS. Full differentiation as judged by morphological changes and red oil $\mathrm{O}$ staining occurred 9 days after changing to MDI induction medium (see Result section).

\section{mRNA isolation}

Upon changing the medium to MDI induction medium, samples were taken each day to measure resistin mRNA expression during differentiation until day 9. Additionally, the cells were exposed to series of TZDs on day 7 for $24 \mathrm{~h}$. For mRNA isolation the medium was aspired and cells were washed with PBS. Then $600 \mu \mathrm{l}$ of $1 \% \beta$-mercaptoethanol in RLT lysis buffer (Qiagen kit) were added, cells were scraped and the lysate was collected and stored at $80^{\circ} \mathrm{C}$ until further analysis.

The purification of RNA was done using the RNeasy mini kit obtained from QIAGEN (Venlo, Netherlands). For the homogenisation of the lysate, a QIAshredder spin column was used (Qiagen, Venlo, Netherlands). In brief the lysate $(600 \mu \mathrm{l})$ was transferred onto a QIAshredder spin column and centrifuged at $14000 \mathrm{rpm}$ for 2 minutes. Then $600 \mu \mathrm{l}$ of $70 \%$ ethanol were added to the flow through of the spin column and the sample was mixed thoroughly. Part of lysate/ethanol mixture was transferred to an RNeasy column and centrifuged at $14000 \mathrm{rpm}$ for $15 \mathrm{sec}$. The flow through was discarded. The rest of the lysate/ethanol mixture were added to the same RNeasy column and centrifuged at $14000 \mathrm{rpm}$ for $15 \mathrm{sec}$. The flow through was discarded. Next RW1 buffer was added to the column and the column was centrifuged at $14000 \mathrm{rpm}$ for $15 \mathrm{~s}$ and the flow through was discarded. Next $500 \mu \mathrm{l}$ of RPE buffer was added to the column and the column was centrifuged at $14000 \mathrm{rpm}$ for $15 \mathrm{~s}$ and the flow through was discarded. The step was repeated one more time with 2 min of centrifugation. The column was transferred to a new tube and RNase-free water (30 $\mu \mathrm{l})$ was added, followed by centrifugation at $14000 \mathrm{rpm}$ for $15 \mathrm{~s}$ to elute total RNA. The concentration of total RNA that was collected in the flow through was measured with a Nanodrop (ND1000, Thermo scientific, Wilmington, Delaware). 


\section{Chapter 2}

\section{Reverse Transcriptase reaction and Real-Time PCR with SYBR green}

To perform the reverse transcriptase (RT) reaction an Omniscript RT kit (Qiagen, Venlo, Netherlands) was used. Total RNA samples were diluted to $0.33 \mu \mathrm{g} / \mu \mathrm{l}$ in RNase-free water. To each sample $2 \mu \mathrm{l}$ 10x buffer RT, $2 \mu \mathrm{d}$ dNTP Mix, $2 \mu 1$ Random Hexamer Primers, $2.5 \mu 1$ RNase Inhibiter, $1 \mu \mathrm{l}$ Omniscript Reverse Transcriptase and $4.5 \mu \mathrm{l}$ of RNase-free water were added and the mixture was incubated for 60 minutes at $37^{\circ} \mathrm{C}$ in an iCycler (Bio-Rad) to obtain cDNA.

The expression of resistin mRNA was measured by real-time quantitative chain polymerase reaction (RT-qPCR) using an iCycler (Bio-Rad). For every reaction $5 \mu \mathrm{l}$ of sample cDNA, 1 $\mu \mathrm{l}$ forward (CTGTGTCCCATCGATGAAGC), $1 \mu \mathrm{l}$ reverse (CTCAAGACTGCTGTGCC TTC) primers specific for mouse resistin, $12.5 \mu \mathrm{l}$ of Sybr green mastermix (Bio-Rad) and 5.5 $\mu \mathrm{l}$ of RNase free water were used. The plate was incubated at $95^{\circ} \mathrm{C}$ for $15 \mathrm{~min}$, and then for 45 cycles each consisting of incubation at $95^{\circ} \mathrm{C}$ for $30 \mathrm{sec}$, at $60^{\circ} \mathrm{C}$ for $30 \mathrm{sec}$, at $72^{\circ} \mathrm{C}$ for 45 sec. This was followed by incubation at $72^{\circ} \mathrm{C}$ for $5 \mathrm{~min}$, at $95^{\circ} \mathrm{C}$ for $1 \mathrm{~min}$ and finally $10 \mathrm{sec}$ for every half degree from $95^{\circ} \mathrm{C}$ to $55^{\circ} \mathrm{C}$ for the melting curve. Hypoxanthine-guanine phosphoribosyltransferase (Hprt) quantitect primer assay by Qiagen was used as invariant endogenous control.

\section{Data analysis}

For the data analysis GraphPad Prism software was used (version 5.00 for windows, GraphPad software, San Diego, USA). The gene expression of resistin was measured with real time PCR. Relative quantification was performed according to the comparative $2^{-\Delta \Delta \mathrm{Ct}}$ method (Pfaffl, 2001). The relative expression was normalized with the Hprt gene, which was taken as a housekeeping gene. The $\Delta \Delta \mathrm{Ct}$ calculation was used as follows $\Delta \Delta \mathrm{Ct}=(\mathrm{Ct}$, target gene $-\mathrm{Ct}$, Hprt $)-\left(\mathrm{Ct}_{\mathrm{c}}\right.$, target gene $-\mathrm{Ct}_{\mathrm{c}}$, Hprt $)$, with $\mathrm{Ct}$ being the number of threshold cycles of the target gene and $\mathrm{Ct}_{\mathrm{c}}$ being the number of threshold cycles of the control, in this case the unexposed cell sample. The results for the gene expression was given as a unitless value through the formula $2^{-\Delta \Delta \mathrm{Ct}}$ (Pfaffl, 2001). For the PPAR $\gamma$ activation and the inhibition of resistin mRNA expression, dose-response curves of the data were used to determine the $\mathrm{EC}_{50}$ and the $\mathrm{IC}_{50}$ values respectively. The correlation between $\mathrm{PPAR} \gamma \mathrm{EC}_{50}$ values and resistin $\mathrm{IC}_{50}$ values between resistin $\mathrm{IC}_{50}$ and MED values was determined by calculating the linear regression $\left(\mathrm{R}^{2}\right)$ and the correlation coefficient (Pearson $\mathrm{r}$ ). 


\section{Results}

\section{Synthesis of TZD model compounds}

Although several TZDs could be commercially obtained many additional TZD model compounds had to be synthesized. The compounds CS-7017, BRL-48482, DRF-2189, 65, 66 and PMT-13 were synthesized according to literature (Buckle et al., 1996; Cantello et al., 1994; Chakrabarti et al., 2002; Lohray et al., 1998; McTernan P. et al., 2003). Purity and identity of the synthetized compounds were analysed by ${ }^{1} \mathrm{H}-\mathrm{NMR}$. Spectral data were identical to the data reported in literature (Buckle et al., 1996; Cantello et al., 1994; Chakrabarti et al., 2002; Lohray et al., 1998; McTernan P. et al., 2003).

\section{PPAR $\gamma$ activation by the TZD model compounds in the PPAR $\gamma-2$ CALUX stable reporter cell line}

For the determination of PPAR $\gamma$ activation by the ligand specific TZDs the recently developed PPAR $\gamma$-2 CALUX cell line (Gijsbers et al., 2011) was used. Figure 2 shows the dose-dependent luciferase induction in the assay upon exposure of the cells to increasing concentrations of four representative TZDs. The results are expressed as percentage of the positive control, rosiglitazone, measured as relative light units (RLUs). The different TZDs show different potencies for PPAR $\gamma$ mediated induction of luciferase activity. From the dose dependent curves using the GraphPad software, the $\mathrm{EC}_{50}$ values for the PPAR $\gamma$ induction by the different TZDs were determined (Table 1). These results reveal that CS-7017 showed the highest potency, reflected by an $\mathrm{EC}_{50}$ value that was 20 fold lower than that for rosiglitazone. CAY-10506 and BRL-48482 were also more potent than rosiglitazone with $\mathrm{EC}_{50}$ values that were respectively 2.3 and 1.3 fold lower than that of rosiglitazone. PMT-13, DRF-2519, 65, 66, CAY-10415, DRF-2189, 5-(4-fluorobenzilidine)-2,4-TZD and englitazone showed 1-500 fold lower potency than rosiglitazone (Table 1). 


\section{Chapter 2}
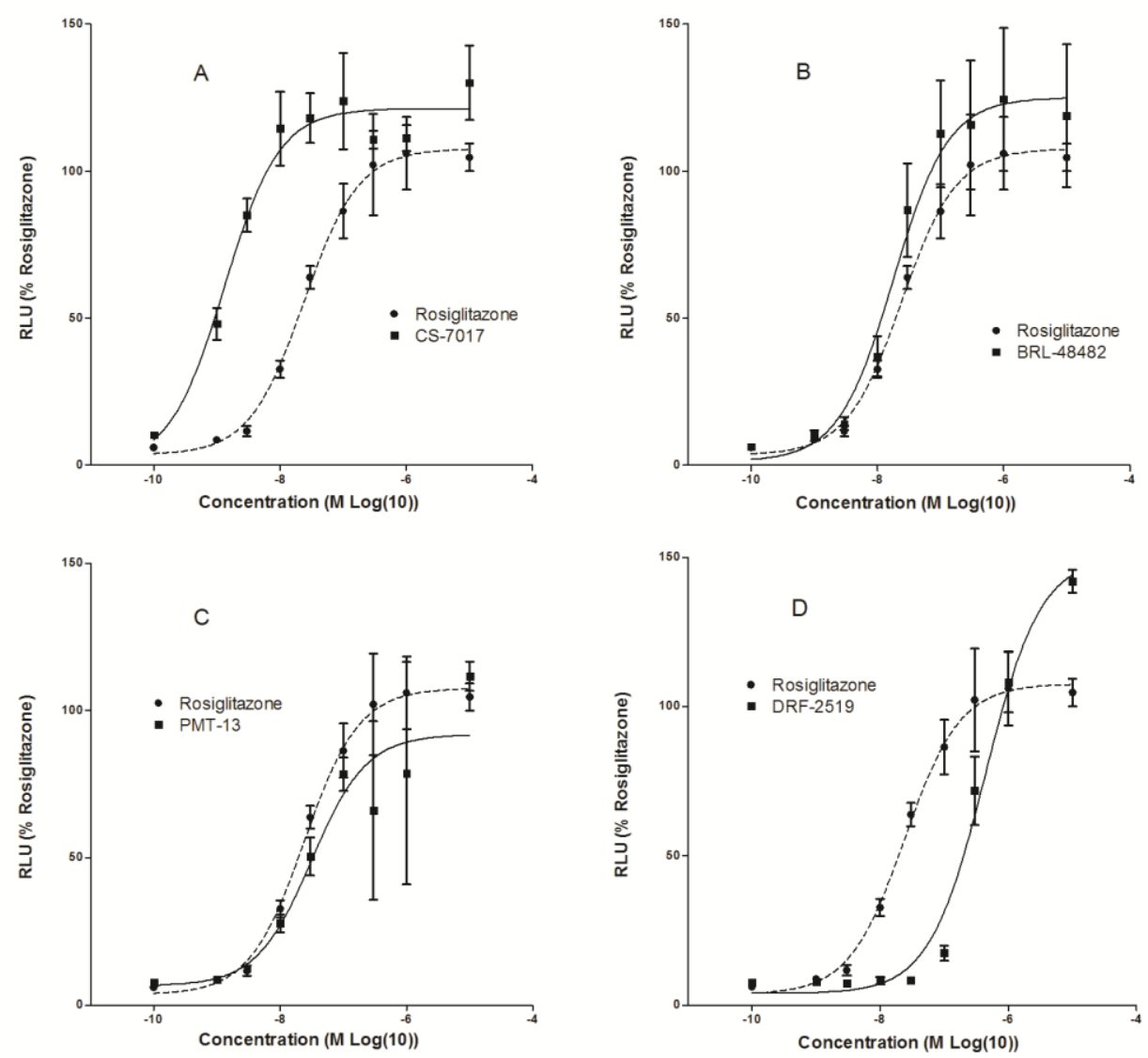

Figure 2. Dose dependent PPAR $\gamma$ activation by four representative TZDs including CS-7017 (A), BRL-48482 (B) and PMT-13 (C), and DRF-2519 (D) expressed as \% of the maximum rosiglitazone response (rosiglitazone, dotted line) which is included for comparison, measured as relative light units.

Table 1. $\mathrm{EC}_{50}$ values of PPAR $\gamma$ activation as tested in this study and in literature, $\mathrm{IC}_{50}$ values of resistin mRNA inhibition in $\mu \mathrm{M}$ calculated using Graphpad software v.5 and minimum effective dose (MED) in vivo from literature.

\begin{tabular}{lllll} 
Thiazolidinedione & $\mathbf{E C}_{\mathbf{5 0}}(\boldsymbol{\mu M})$ & $\mathbf{I C}_{\mathbf{5 0}}(\boldsymbol{\mu M})$ & $\begin{array}{l}\mathbf{E C}_{\mathbf{5 0}}(\boldsymbol{\mu M}) \\
\text { Literature }\end{array}$ & MED $(\boldsymbol{\mu m o l ~ k g - 1})$ \\
\hline Englitazone & 13.39 & - & 13 & - \\
DRF-2519 & 0.435 & 1.07 & - & - \\
CAY-10506 & 0.0106 & 0.0585 & 10 & - \\
CAY-10415 & 4.88 & 0.198 & - & - \\
65 & 0.0786 & 0.187 & 0.14 & 300 \\
66 & 0.342 & 0.0521 & 0.19 & 300 \\
5-(4- & 11.9 & - & - & - \\
fluorobenzylidine) & & & & - \\
-2,4-TZD & & & & - \\
DRF-2189 & 0.798 & 2.28 & - & - \\
PMT-13 & 0.0314 & 0.00584 & 0.01 & 3 \\
CS-7017 & 0.00132 & 0.000595 & 0.0002 & 3 \\
BRL-48482 & 0.0178 & 0.00246 & 0.013 & 3000 \\
Rosiglitazone & 0.024 & 0.0262 & 0.01 & 300 \\
Ciglitazone & - & 7.553 & 3 & 0.69 \\
Pioglitazone & 0.60 & 4.59 & &
\end{tabular}




\section{Optimization of the 3T3-L1 adipocytes differentiation}

Figure 3 shows the morphology of the 3T3-L1 preadipocytes during the differentiation process to adipocytes followed by microscopy. Changes in the morphology of the cells started at day 3. Lipid droplets started to be formed after day 6 and full maturation to adipocytes was achieved at day 9. For the sample at day 9 the presence of lipid droplets was confirmed by staining with Red Oil O which is a lipid specific stain (Figure 3E). Figure 4 shows the resistin mRNA detected in the cells from day 0 to 9 during the differentiation of pre-adipocytes to adipocytes. From these data it follows that resistin mRNA levels increase upon increased differentiation of the cells with levels increased from day 7 onwards. Based on these results it was decided to expose the cells to the various TZDs from day 7 to 8 and detect possible effects on resistin expression levels at day 8.

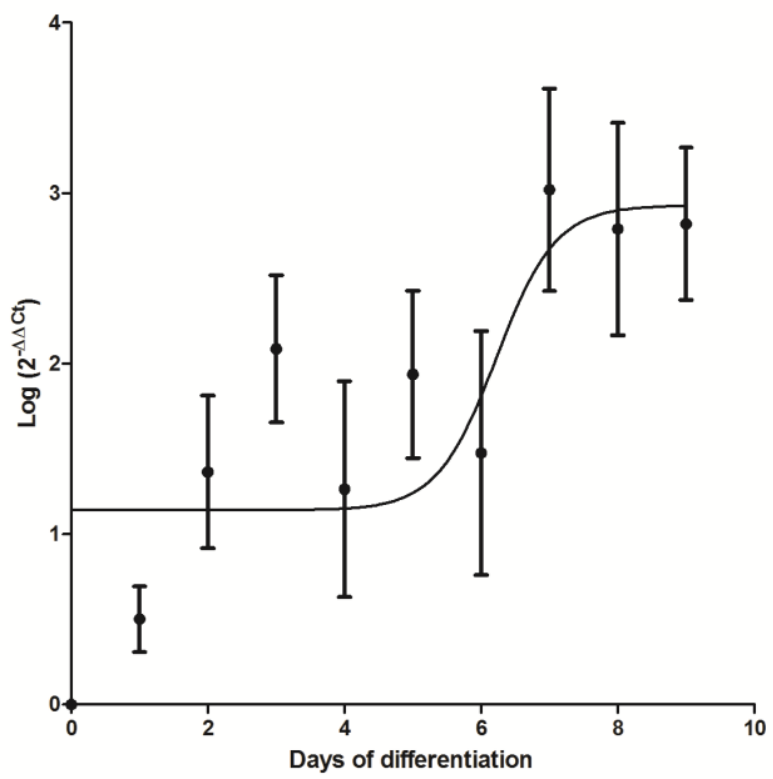

Figure 4. Resistin mRNA expression during differentiation from day 0 to day 9 expressed as $\log \left(2^{-\Delta \Delta C t}\right)$, normalized with undifferentiated cells of day 0 .

\section{Resistin downregulation by the TZD model compounds in 3T3-L1 adipocytes}

Figure 5 shows the dose dependent change of resistin gene expression as quantified by qPCR after the exposure of the cells to a series of TZDs. From the results presented, it follows that resistin gene expression is inhibited by TZDs. The $\mathrm{IC}_{50}$ values for the TZD mediated resistin downregulation in the 3T3-L1 adipocytes were derived from these dose-dependent curves using the Graphpad software and are shown in Table 1. The TZDs clearly differed in their potency for resistin downregulation in the 3L3-L1 adipocytes. CS-7017 showed the highest 


\section{Chapter 2}
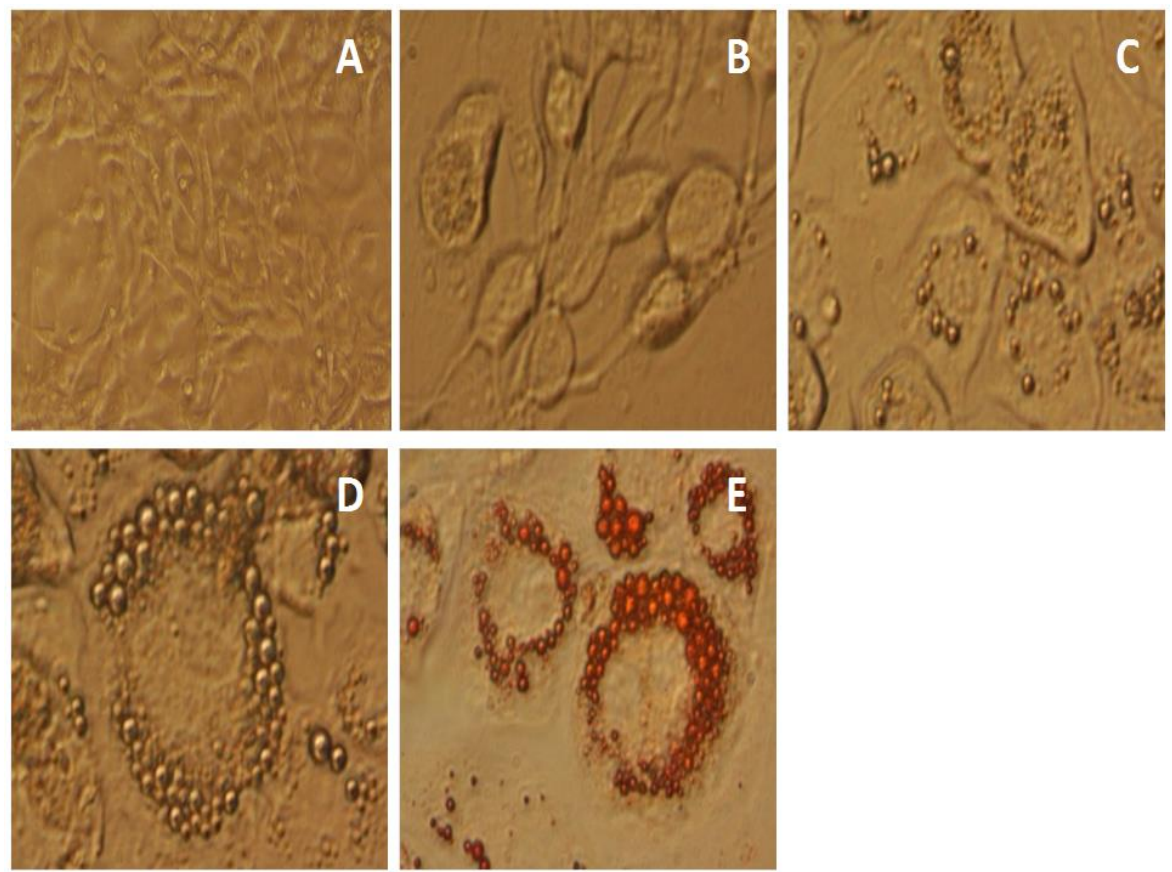

Figure 3. Microscopic pictures of 3T3-L1 cells differentiating into mature adipocytes at day 0 (A), day 3 (B), day 6 (C) and day 9 (D) during differentiation. The presence of lipids on day 9 was confirmed by the Oil Red O staining $(\mathrm{E})$.

potency for inhibiting resistin gene expression reflected by an $\mathrm{IC}_{50}$ that was 44 fold lower than that obtained for rosiglitazone, a known inhibitor of resistin gene expression. BRL-48482, PMT-13, were also more potent than rosiglitazone with $\mathrm{IC}_{50}$ values that were respectively 10 and 4.5 fold lower than that of rosiglitazone. CAY-10415, CAY-10506, 65, 66, DRF-2519 and DRF-2189 showed 2-80 fold lower potency than rosiglitazone. Englitazone and 5-(4fluorobenzilidene)-2,4-TZD were not able to inhibit resistin gene expression in the 3T3-L1 adipocytes. Using the MTT assay it was established that at the concentrations tested the TZDs did not affect cell viability.

Figure 6 shows the correlation between the calculated $\mathrm{EC}_{50}$ values for PPAR $\gamma$ activation in the reporter gene assay and the $\mathrm{IC}_{50}$ values for resistin downregulation in the mouse adipocytes. A significant correlation $(\mathrm{p}<0.05)$ of $\mathrm{R}^{2}=0.693$ is obtained. The Pearson correlation coefficient amounts to $r=0.832$. Figure 7 shows the correlation between the calculated $\mathrm{IC}_{50}$ value for resistin downregulation and the MED for the antihyperglycemic activity in vivo of six TZDs for which these MED data were available (Willson et al., 1996). The correlation between resistin downregulation and the MED for antihyperglycemic activity revealed an $\mathrm{R}^{2}=0.71(\mathrm{p}<0.05)$ and a Pearson correlation coefficient of $\mathrm{r}=0.877$. 

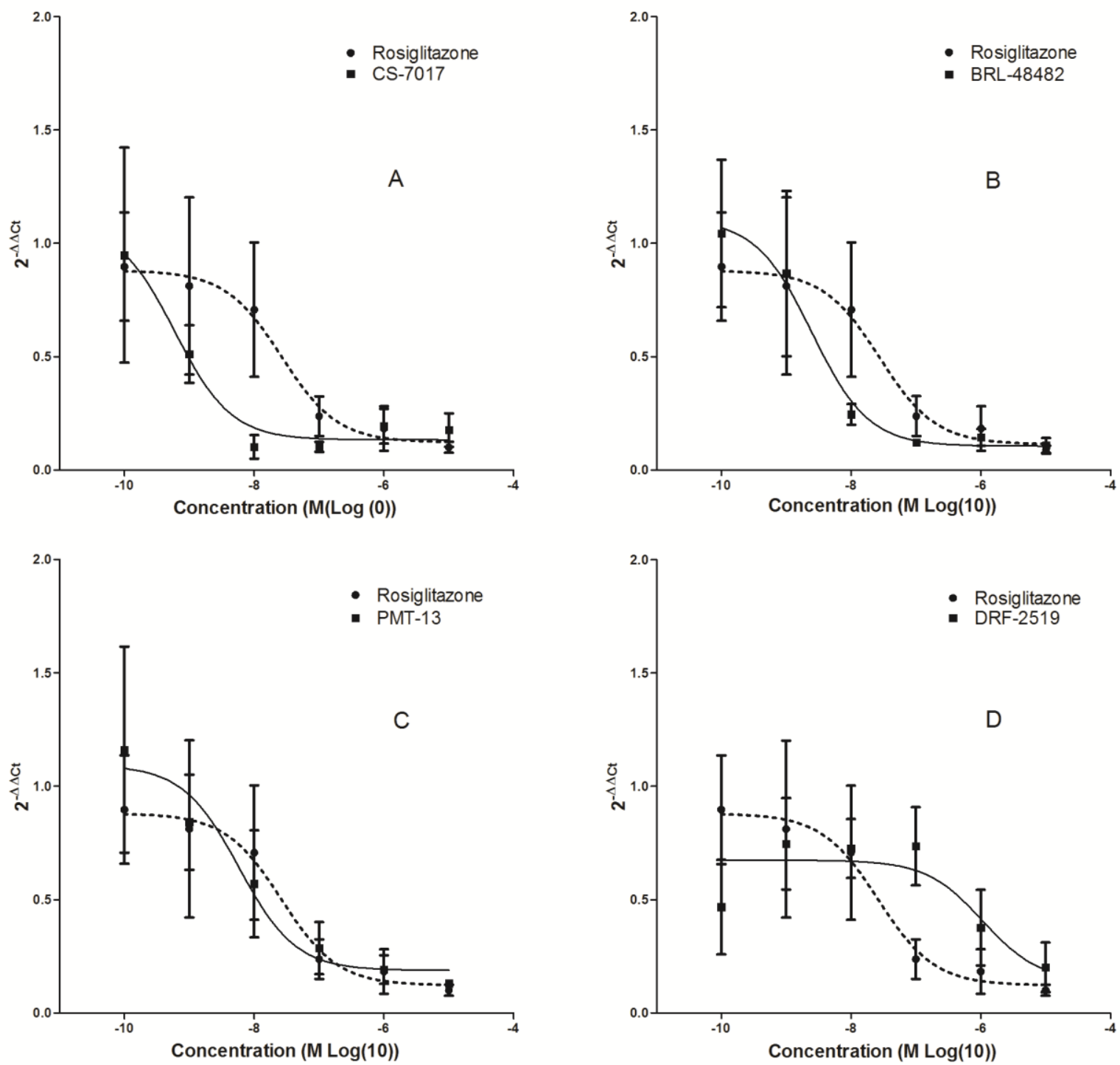

Figure 5. Inhibition of resistin mRNA expression in 3T3-L1 mouse adipocytes by four representative TZDs, CS7017 (A), BRL-48482 (B) PMT-13 (C) and DRF-2519 (D) expressed as unitless value from the formula of 2$\Delta \Delta \mathrm{CT}$. All figures also present the curve for rosiglitazone (dotted line) used as the reference compound. The experiment was repeated 3 times. 


\section{Chapter 2}

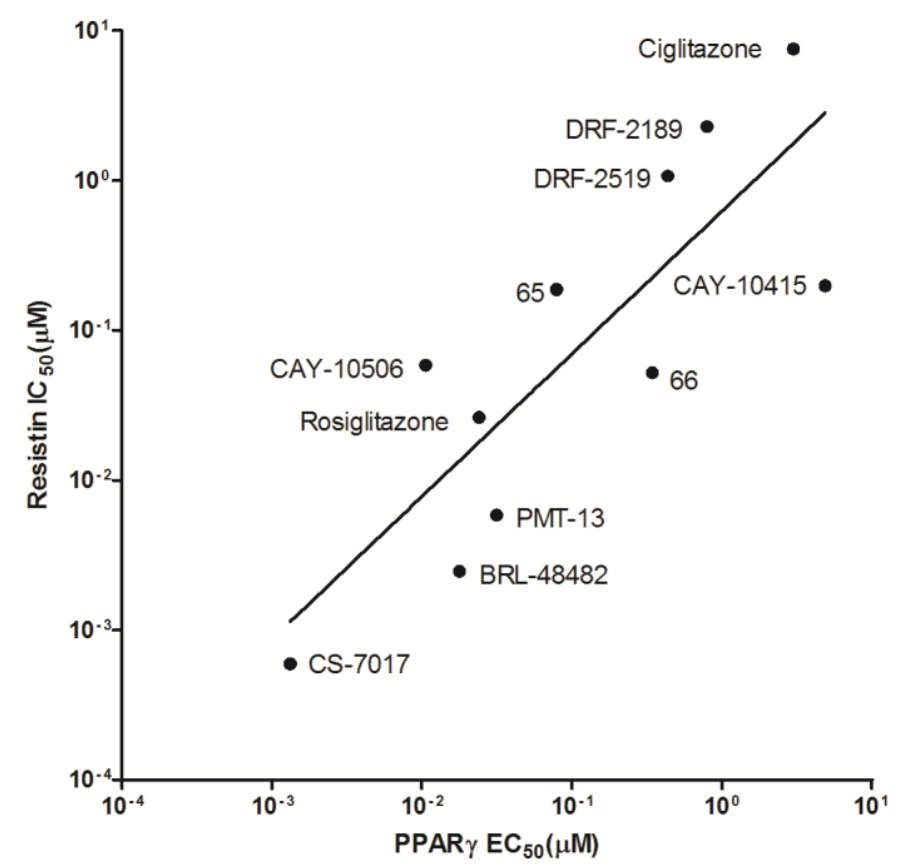

Figure 6. Correlation between $\mathrm{EC}_{50}$ values for PPAR $\gamma$ activation in a reporter gene assay and $\mathrm{IC}_{50}$ values for inhibition of resistin expression in mouse adipocytes. A significant correlation of $\mathrm{R}^{2}=0.693$ with $\mathrm{p}<0.05$ was obtained. The Pearson correlation coefficient was calculated $\mathrm{r}=0.832$.

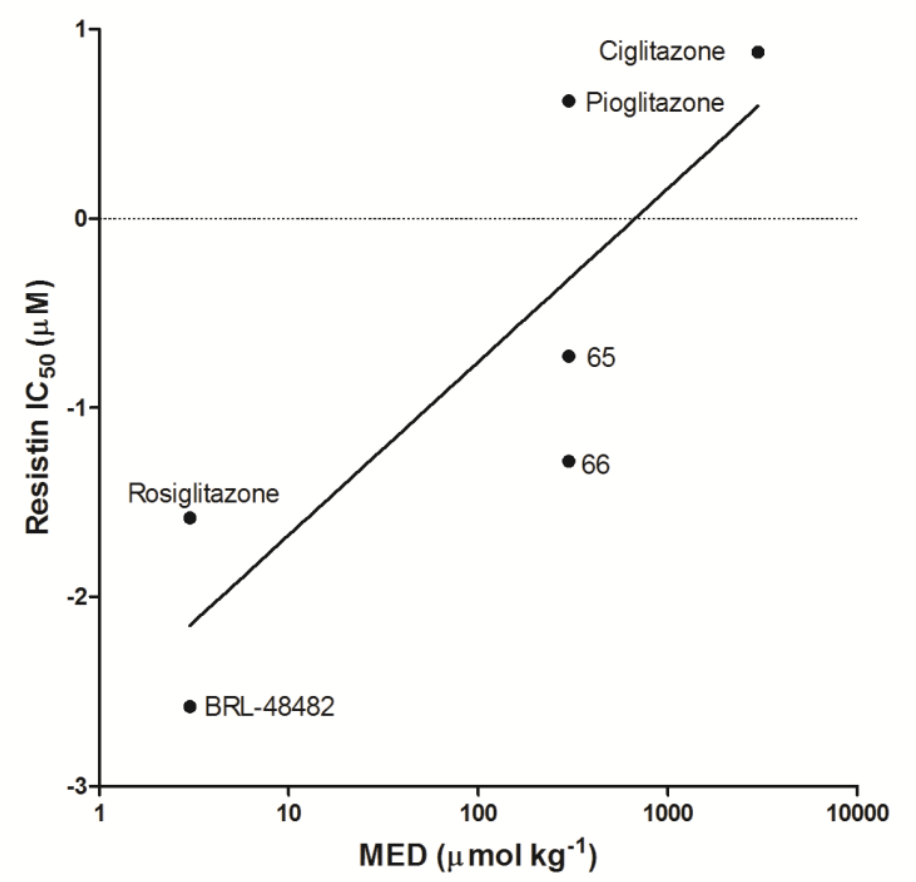

Figure 7. Correlation between $\mathrm{IC}_{50}$ for downregulation of resistin in mouse adipocytes and the minimum effective dose (MED) for antihyperglycemic activity found in vivo in a mouse model (Willson et al., 1996). A significant correlation of $\mathrm{R}^{2}=0.71$ with $\mathrm{p}<0.05$ was obtained. The Pearson correlation coefficient was calculated $\mathrm{r}=0.877$. 


\section{Discussion}

The aim of the present study was to investigate whether resistin downregulation in mouse 3T3-L1 adipocytes could provide a suitable endpoint for testing new antihyperglycemic drugs, as an alternative for PPAR $\gamma$ agonist activity. To this end it was investigated if for a series of related TZDs the $\mathrm{EC}_{50}$ for activation of PPAR $\gamma$ detected in a recently developed reporter gene assay would correlate with their potential for resistin downregulation in mouse adipocytes, and subsequently if the $\mathrm{IC}_{50}$ values for resistin downregulation would correlate with the MED for antihyperglycemic activity in a mouse model reported in the literature (Willson et al., 1996).

For fourteen TZDs their potency to activate PPAR $\gamma$ in the PPAR $\gamma$-2 CALUX assay and their potency to down regulate resistin in 3T3-L1 adipocytes was quantified. The $\mathrm{EC}_{50}$ values for PPAR $\gamma$ mediated gene expression obtained using the newly developed PPAR $\gamma-2$ stable reporter cell line were comparable to the $\mathrm{EC}_{50}$ values reported in the literature for PPAR $\gamma$ agonist activity of some the TZDs using other PPAR $\gamma$ reporter gene assays (Imran et al., 2007; Willson et al., 1996). The only difference between the data previously reported and those obtained in the present study were the results obtained for CAY-10506 that showed a 1000 fold lower $\mathrm{EC}_{50}$ value in the present study than reported in literature.

Rosiglitazone is a known inhibitor of resistin gene expression (Daquinag et al., 2011; Haugen et al., 2001; Rajala et al., 2003) and the results of the present study reveal that also other TZDs can reduce resistin expression levels. The TZDs differ in their potency to inhibit resistin gene expression in the mouse adipocytes.

A comparison between the $\mathrm{EC}_{50}$ values for PPAR $\gamma$ activation and the $\mathrm{IC}_{50}$ values for resistin downregulation revealed a significant correlation ( $\mathrm{p}<0.05$ ) of $\mathrm{R}^{2}=0.693$ (Figure 6). The calculated correlation coefficient showed a Pearson $\mathrm{r}$ of 0.832 . This correlation between $\mathrm{EC}_{50}$ for PPAR $\gamma$ agonist activity and the $\mathrm{IC}_{50}$ for resistin dowregulation may reflect that the resistin downregulation is PPAR $\gamma$ mediated. This would be in line with the findings of Steppan et al. (2001) who reported that resistin is downregulated by the TZDs upon PPAR $\gamma$ activation.

In a subsequent analysis a direct comparison between the $\mathrm{IC}_{50}$ for inhibition of resistin expression and the MED for antihyperglycemic activity of a series of six TZDs in a mouse model (Willson et al., 1996) was performed. This comparison also revealed, a significant correlation as shown in Figure $7\left(\mathrm{R}^{2}=0.71, \mathrm{p}<0.05\right)$. The correlation coefficient showed a 


\section{Chapter 2}

Pearson $\mathrm{r}$ of $0.877(\mathrm{p}<0.05)$. These correlations indicate that PPAR $\gamma$ mediated downregulation of resistin might inhibit its negative effects and promote insulin sensitivity, and that downregulation of resistin in mouse adipocytes provides an adequate bioassay, in addition to PPAR $\gamma$ activation, for the screening of newly developed antihyperglycemic compounds. Because of the higher throughput of the PPAR $\gamma$ the resistin downregulation assays seems most suitable to be used as a second tier in a tiered screening strategy.

\section{Acknowledgement}

This research was financially supported by the Dutch Ministry of Economic Affairs, Agriculture and Innovation, Grant Pieken in de Delta - Healthy ageing project, PID082022.

\section{References}

Barnes, K. M. and J. L. Miner (2009). "Role of resistin in insulin sensitivity in rodents and humans." Curr Protein Pept Sci 10(1): 96-107.

Berger, J. and D. E. Moller (2002). "The mechanisms of action of PPARs." Annu Rev Med 53: 409-435.

Boden, G. (1997). "Role of fatty acids in the pathogenesis of insulin resistance and NIDDM." Diabetes 46(1): 3-10.

Boden, G., et al. (2005). "Thiazolidinediones upregulate fatty acid uptake and oxidation in adipose tissue of diabetic patients." Diabetes 54(3): 880-885.

Buckle, D. R., et al. (1996). "Non thiazolidinedione antihyperglycaemic agents .1. alphaheteroatom substituted beta-phenylpropanoic acids." Bioorg Med Chem Lett 6(17): 21212126.

Cantello, B. C., et al. (1994). "[[omega-(Heterocyclylamino)alkoxy]benzyl]-2,4thiazolidinediones as potent antihyperglycemic agents." Journal of Medicinal Chemistry 37(23): 3977-3985.

Chakrabarti, R., et al. (2002). "PMT13, a pyrimidone analogue of thiazolidinedione improves insulin resistance-associated disorders in animal models of type 2 diabetes." Diabetes Obes Metab 4(5): 319-328.

Daquinag, A. C., et al. (2011). "An isoform of decorin is a resistin receptor on the surface of adipose progenitor cells." Cell Stem Cell 9(1): 74-86.

Ferrannini, E. (1992). "The insulin resistance syndrome." Curr Opin Nephrol Hypertens 1(2): 291-298.

Gijsbers, L., et al. (2011). "Stable reporter cell lines for peroxisome proliferator-activated receptor gamma (PPARgamma)-mediated modulation of gene expression." Anal Biochem 414(1): 77-83. 
Hartman, H. B., et al. (2002). "Mechanisms regulating adipocyte expression of resistin." J Biol Chem 277(22): 19754-19761.

Haugen, F., et al. (2001). "Inhibition by insulin of resistin gene expression in 3T3-L1 adipocytes." FEBS Lett 507(1): 105-108.

Hussain, R. F., et al. (1993). "A new approach for measurement of cytotoxicity using colorimetric assay." J Immunol Methods 160(1): 89-96.

Imran, M., et al. (2007). "Recent thiazolidinediones as antidiabetics." Journal of Scientific \& Industrial Research 66(2): 99-109.

Lazar, M. A. (2007). "Resistin- and Obesity-associated metabolic diseases." Horm Metab Res 39(10): 710-716.

Lehrke, M. and M. A. Lazar (2005). "The many faces of PPARgamma." Cell 123(6): 993999.

Lohray, B. B., et al. (1998). "Novel euglycemic and hypolipidemic agents. 1." Journal of Medicinal Chemistry 41(10): 1619-1630.

McTernan, P. G., et al. (2003). "Resistin and type 2 diabetes: regulation of resistin expression by insulin and rosiglitazone and the effects of recombinant resistin on lipid and glucose metabolism in human differentiated adipocytes." J Clin Endocrinol Metab 88(12): 60986106.

McTernan, P. G., et al. (2002). "Increased resistin gene and protein expression in human abdominal adipose tissue." J Clin Endocrinol Metab 87(5): 2407.

Okuno, A., et al. (1998). "Troglitazone increases the number of small adipocytes without the change of white adipose tissue mass in obese Zucker rats." J Clin Invest 101(6): 13541361.

Patel, L., et al. (2003). "Resistin is expressed in human macrophages and directly regulated by PPAR gamma activators." Biochem Biophys Res Commun 300(2): 472-476.

Patel, S. D., et al. (2004). "Disulfide-dependent multimeric assembly of resistin family hormones." Science 304(5674): 1154-1158.

Pfaffl, M. W. (2001). "A new mathematical model for relative quantification in real-time RTPCR." Nucleic Acids Res 29(9): e45.

Rajala, M. W., et al. (2003). "Adipose-derived resistin and gut-derived resistin-like moleculebeta selectively impair insulin action on glucose production." J Clin Invest 111(2): 225230 .

Rajala, M. W., et al. (2004). "Regulation of resistin expression and circulating levels in obesity, diabetes, and fasting." Diabetes 53(7): 1671-1679.

Reitman, M. L., et al. (2000). "Lipoatrophy revisited." Trends Endocrinol Metab 11(10): 410416.

Saltiel, A. R. and C. R. Kahn (2001). "Insulin signalling and the regulation of glucose and lipid metabolism." Nature 414(6865): 799-806. 


\section{Chapter 2}

Sanchez-Solana, B., et al. (2012). "Mouse Resistin Modulates Adipogenesis and Glucose Uptake in 3T3-L1 Preadipocytes Through the ROR1 Receptor." Molecular Endocrinology 26(1): 110-127.

Shimazaki, N., et al. (2008). "Anti-tumour activity of CS-7017, a selective peroxisome proliferator-activated receptor gamma agonist of thiazolidinedione class, in human tumour xenografts and a syngeneic tumour implant model." European Journal of Cancer 44(12): 1734-1743.

Steppan, C. M., et al. (2001). "The hormone resistin links obesity to diabetes." Nature 409(6818): 307-312.

Stofkova, A. (2010). "Resistin and visfatin: regulators of insulin sensitivity, inflammation and immunity." Endocr Regul 44(1): 25-36.

Tarcin, O., et al. (2007). "Insulin resistance, adipocyte biology, and thiazolidinediones: a review." Metab Syndr Relat Disord 5(2): 103-115.

Tarkowski, A., et al. (2010). "Resistin competes with lipopolysaccharide for binding to tolllike receptor 4." J Cell Mol Med 14(6B): 1419-1431.

Tontonoz, P. and B. M. Spiegelman (2008). "Fat and beyond: the diverse biology of PPARgamma." Annu Rev Biochem 77: 289-312.

Willson, T. M., et al. (1996). "The structure-activity relationship between peroxisome proliferator-activated receptor gamma agonism and the antihyperglycemic activity of thiazolidinediones." Journal of Medicinal Chemistry 39(3): 665-668.

Yki-Jarvinen, H. (2004). "Thiazolidinediones." N Engl J Med 351(11): 1106-1118. 




\title{
Chapter 3
}

\section{Toll-like receptor 4 (TLR-4) signaling as a method for detecting resistin antagonism.}

\author{
A. Sotiriou \\ H. Xiong \\ L.H.J. De Haan \\ J. Vervoort
}

I.M.C.M. Rietjens 


\title{
Chapter 3
}

\begin{abstract}
The present study investigated whether a reporter assay detecting Toll-like receptor 4 (TRL-4) mediated gene expression could be a possible bioassay to detect candidate drugs able to inhibit resistin mediated effects. This was done because inhibition of resistin mediated activities may prove a novel target for development of antidiabetic drugs. To detect activation of TLR-4 mediated gene expression and enable detection of possible antagonists for resistin mediated activation of the receptor, a TLR-4 reporter gene cell line (HEK-Blue-TLR-4) was applied. The results obtained reveal that resistin, like the natural ligand lipopolysaccharide (LPS) is able to activate TLR-4 mediated gene expression with $\mathrm{EC}_{50}$ values for LPS and resistin amounting to respectively $0.05 \mu \mathrm{g} / \mathrm{ml}$ and $5 \mu \mathrm{g} / \mathrm{ml}$. Resistin was also shown to compete with LPS for binding to TLR-4. The LPS or resistin dependent induction of TLR-4 mediated gene expression was inhibited by two known TLR-4 inhibitors including, LPS-RS and Polymyxin B. It is concluded that the TLR-4 reporter gene cell line provides a novel tool to detect resistin antagonists and may be of use as a new target to screen for a possible new type of antidiabetic drugs.
\end{abstract}




\section{Introduction}

The causes of Type 2 diabetes mellitus (T2DM) are impaired insulin production and the inability of insulin sensitive organs to take up glucose, a state that is called insulin resistance. Insulin resistance has been correlated with inflammation (Boden, 1997; Lazar, 2007). Several inflammatory and proinflammatory factors, including resistin, have been identified in obese patients with T2DM (Spanger et al., 2003; Pradhan et al., 2001; Nagaev et al., 2006; Schwartz et al., 2011). Current drugs for the treatment of T2DM mainly originate from the group of thiazolidinediones, and extensive drug development programmes focusing on activation of PPAR $\gamma$ mediated gene expression as an important characteristic of the thiazolidinediones (Willson et al., 1996) have been or are being executed. In the search for additional and alternative targets for treatment of T2DM the hormone resistin has been identified.

Resistin has been associated with the pathogenesis of obesity which is directly connected to T2DM (Lazar et al., 2007; Owecki et al., 2011; Benomar et al., 2016). Resistin is a 12.5 kDa polypeptide and belongs to the resistin-like molecule hormone family (Steppan et.al., 2001, Patel et al., 2004). In rodents and humans, high levels of resistin are correlated with obesity and impaired insulin resistance (Stappan et al., 2001; Owecki et al., 2011). Consequently inhibition of resistin activity using anti-resistin antibodies was shown to improve insulin sensitivity and glucose homeostasis (Steppan et. al., 2001). Nonetheless the receptor mediating these downstream effects on insulin sensitivity is not yet identified (Barnes and Miner, 2009; Daquinag et al., 2011; Haugen et al., 2001; McTernan P. et al., 2003; SanchezSolana et al., 2012; Tarkowski et al., 2010).

It has been shown that resistin can be a ligand towards the Toll-like receptor 4 (TLR-4) and that resistin is able to compete with the natural ligand, which is lipopolysaccharide (LPS), for binding to the TLR-4 (Poltorak et al., 1998; Qureshi et al., 1999; Hoshino et al., 1999; Tarkowski et al., 2010). In these studies antibodies against TLR-4 abolished resistin binding to human leucocytes and inhibited cytokine production by peripheral blood mononuclear cells in response to resistin stimulation (Tarkowski et al., 2010). Activation of the TLR-4 receptor was also shown to play a significant role in the activity of the innate immune system against microbial pathogens and in the induction of adaptive immune responses (Kawai and Akira, 2006; Medzhitov, 2001). It was also shown that TLR-4 detects lipopolysaccharide (LPS) (Tarkowski et al., 2010). Additionally insulin sensitivity was shown to be suppressed by resistin following its binding to TLR-4 (Benomar et al., 2012). Resistin signals were shown to 


\section{Chapter 3}

lead to the activation of pro-inflammatory pathways and the induction of insulin resistance through the regulation of several mediators, such as adaptor protein MyD88, Toll/interleukin1 receptor domain-containing adaptor proteins (TIRAP), Jun NH2-terminal kinase (JNK) and p38 mitogen activated protein kinase (MAPK) (Benomar et al., 2012). More recent studies on TLR-4 knockout mice and neuronal cells expressing TLR-4 siRNAs showed no effects of resistin and revealed a novel mechanism of insulin resistance caused by central resistin-TLR4 pathway where adiponectin is inhibited promoting FGF21 resistance (Benomar et al., 2016)

All the above point at a possible role of resistin mediated activation of TLR- 4 in the activation of inflammatory pathways and the induction of insulin resistance, important responses in T2DM. Given the possible role of resistin mediated activation of TLR-4 in these T2DM related responses it would be of interest to develop a method to detect possible inhibition of resistin induced inflammation and insulin resistance through TLR-4 as a novel target in drug development. Such an assay would allow identification of resistin induced TLR-4 signaling antagonists and or inhibitors that could be considered as possible novel compounds for treatment and or prevention of T2DM. The aim of the present study was to investigate if the TLR-4 reporter gene cell line (HEK-Blue-TLR-4) can be used to detect resistin mediated activation of TLR-4 as well as its inhibition by possible antagonists and or inhibitors. To this end we tested the activity of LPS, a natural ligand for TLR-4, and resistin in this TLR-4 reporter gene cell line. We also investigated whether the assay could detect inhibition of LPS or resistin mediated activation of TLR-4 by two known inhibitors of TLR-4 signaling.

\section{Materials and methods}

\section{Inhibitors and resistin}

Two known, commercially available, inhibitors of TLR-4, LPS-RS and Polymyxin B were purchased from InvivoGen (Toulouse, France). Human resistin was purchased from Bioconnect B.V. (Huissen, Netherlands).

\section{HEK-Blue-TLR-4 cells.}

The reporter gene cell line HEK-Blue-TLR4 was purchased from InvivoGen (Toulouse, France). According to the manufacturer these cells are designed to study human TLR-4 activation by monitoring the activation of NF- $\mathrm{BB}$. More specifically this cell line was generated by transfecting the hTLR-4 gene, the MD-2/CD14 co-receptor genes and a secreted embryonic alkaline phosphatase (SEAP) reporter gene into HEK293 cells. The SEAP reporter 
gene was placed under the control of an IL-20 p40 minimal promoter fused to five NF- $\kappa \mathrm{B}$ and AP-1 binding sites. Consequently any stimulation with TLR-4 ligand would activate NF- $\mathrm{B}$ and AP-1 which would induce the production of SEAP (HEK-Blue ${ }^{\text {TM }}$ hTLR4, InvivoGen). Furthermore SEAP levels were detected with QUANTI-Blue ${ }^{\mathrm{TM}}$ (InvivoGen) which is an alkaline phosphatase detection medium.

\section{Reporter gene assay}

Briefly, cells were maintained in Dulbecco's modified Eagle's medium (DMEM) supplemented with $4.5 \mathrm{~g} / \mathrm{l}$ glucose, $10 \%$ fetal calf serum (FCS, Invitrogen), $50 \mathrm{U} / \mathrm{ml}$ penicillin (Invitrogen), $50 \mu \mathrm{g} / \mathrm{ml}$ streptomycin (Invitrogen), $100 \mu \mathrm{g} / \mathrm{ml}$ Normocin ${ }^{\mathrm{TM}}$ (InvivoGen) and 2 mM L-glutamine (Gibco by life technologies, Netherlands). The growth medium was renewed 2 times per week. Cells were not cultured above 20 passages according to manufacturer's directions.

For testing the compounds $200 \mu \mathrm{l}$ of a cell suspension were plated in 96 well plates at a concentration of $1.4 \times 10^{5}$ cells $/ \mathrm{ml}$. The medium that was used for the testing was supplemented with heat inactivated $\left(56{ }^{\circ} \mathrm{C}\right.$ for $\left.30 \mathrm{~min}\right)$ FCS. TLR-4 signaling was induced according to the protocol provided by the supplier by exposing the cells first to LPS $(0.05$ $\mu \mathrm{g} / \mathrm{ml})$. Simultaneously the exposed cells were exposed to the two TLR-4 inhibitors at final concentrations varying from $3 \mathrm{ng} / \mathrm{ml}$ to $10 \mu \mathrm{g} / \mathrm{ml}$ for LPS-RS and $10 \mathrm{ng} / \mathrm{ml}$ to $70 \mu \mathrm{g} / \mathrm{ml}$ for polymyxin $b$ to inhibit the LPS induced signaling. Plates were incubated at $37{ }^{\circ} \mathrm{C}$ in $5 \% \mathrm{CO}_{2}$ for $22 \mathrm{~h}$. Similarly, TLR-4 signaling was induced by exposing cells to resistin $(5 \mu \mathrm{g} / \mathrm{ml})$ and then simultaneously cells were exposed to the two inhibitors in concentrations ranged as mentioned above.

\section{Quanti-Blue $^{\mathrm{TM}}$ colorimetric enzyme activity assay}

For the detection of the activity of the reporter gene SEAP, $180 \mu 1$ per well Quanti-Blue ${ }^{\mathrm{TM}}$ and $20 \mu \mathrm{l}$ of the supernatant of the exposed cells were incubated for $2 \mathrm{~h}$ at $37{ }^{\circ} \mathrm{C}$ with $5 \% \mathrm{CO}_{2}$. The SEAP activity was measured by measuring the formation of the blue reaction product using a spectrophotometer (Molecular Device, USA) at $620 \mathrm{~nm}$.

\section{MTT assay}

The MTT assay was used to test if there was a possible cytotoxic effect of the compounds at the concentrations tested. In brief, $20 \mu 1$ of 3-(4,5-dimethylthiazol-2-yl)-2,5- 


\section{Chapter 3}

diphenyltetrazolium bromide (MTT) diluted in PBS were added to the HEK-Blue-TLR4 cells exposed to the TLR-4 inhibitors at the same concentrations as in the inhibition experiments. Plates were incubated for $22 \mathrm{~h}$ at $37{ }^{\circ} \mathrm{C}$ with $5 \% \mathrm{CO}_{2}$. Then medium was removed and $100 \mu \mathrm{l}$ DMSO (Merck, Germany) were added to lyse the cells and dissolve the formazan crystals. Plates were shaken for $15 \mathrm{~min}$ to allow the blue/violate formazan crystals to dissolve. The OD was measured at $562 \mathrm{~nm}$ and $620 \mathrm{~nm}$. The results were expressed as percentage of the lowest concentration.

All experiments were performed in triplicate and data are expressed as the percentage of the lowest concentrations of the average of three experiments. $\mathrm{IC}_{50}$ values were determined using the Graphpad Prism 5 software. The software uses a non-linear regression fit described by the equation:

$$
\mathrm{Y}=\text { Bottom }+(\text { Top-Bottom }) /\left(1+10^{\wedge}\left(\left(\log \mathrm{IC}_{50}-\mathrm{X}\right) * \text { HillSlope }\right)\right)
$$

\section{Results}

\section{Resistin induces TLR-4 signaling in HEK-blue TLR-4 cells}

The first step was to verify resistin's TLR-4 inducing ability in HEK-blue TLR-4 cells and compare it with the induction by LPS, the natural ligand of TLR-4. Figures 1A and 1B show the LPS induced TLR-4 signaling and resistin induced TLR-4 signaling. From these data it can be derived that both compounds are able to induce TLR-4 mediated gene expression with the $\mathrm{EC}_{50}$ of LPS being $0.05 \mu \mathrm{g} / \mathrm{ml}$ and 100 fold lower that the $\mathrm{EC}_{50}$ of resistin which amounted to $5 \mu \mathrm{g} / \mathrm{ml}$.
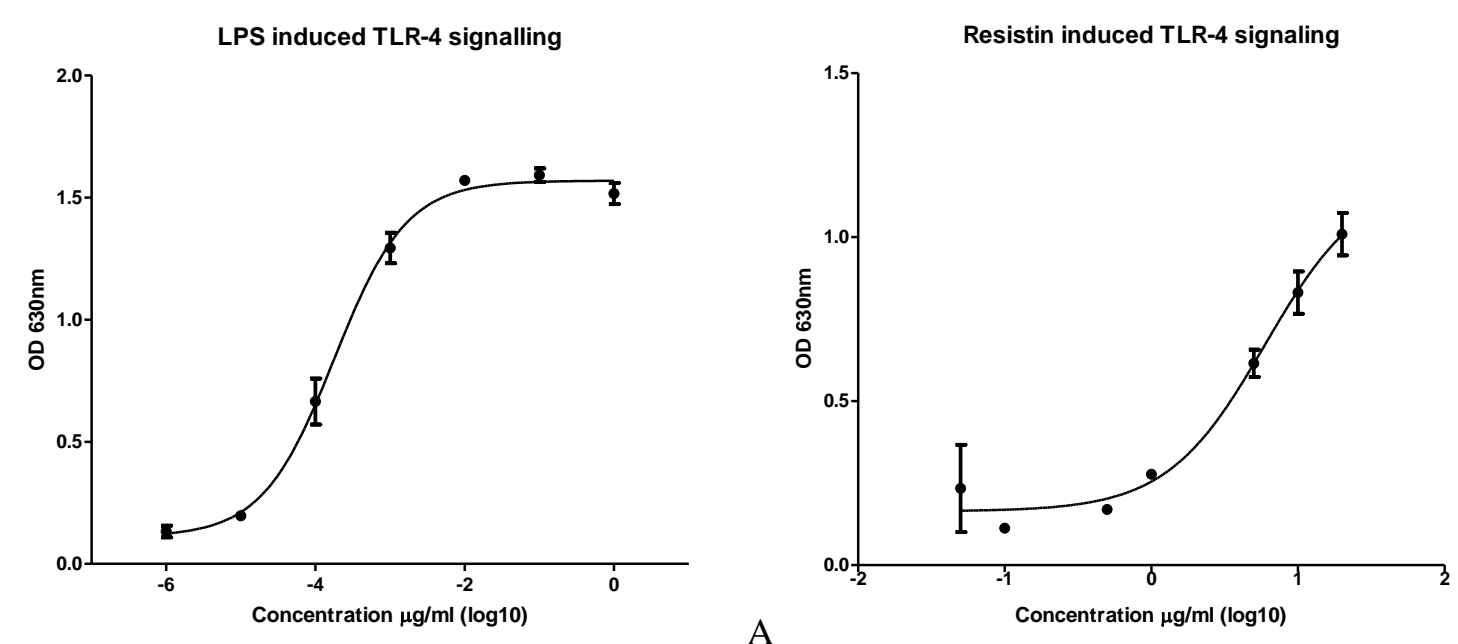

Figure 1. LPS induced TLR-4 signaling by LPS (A) and resistin (B). 


\section{Viability of the cells upon exposure to the TRL-4 inhibitors}

In a next step it was investigated whether the TRL-4 inhibitors to be tested would affect viability of the HEK-Blue-TLR4 cells. This was done to enable selection of non-cytotoxic concentrations to be tested in the TLR-4 reporter gene assay and/or correct for cytotoxicity if needed. From these data it can be derived that LPS-RS and Polymyxin B did not affect cell viability up to the highest concentrations tested (Figures 2A, 2B). This can be taken into account when analyzing results from the reporter gene assay.
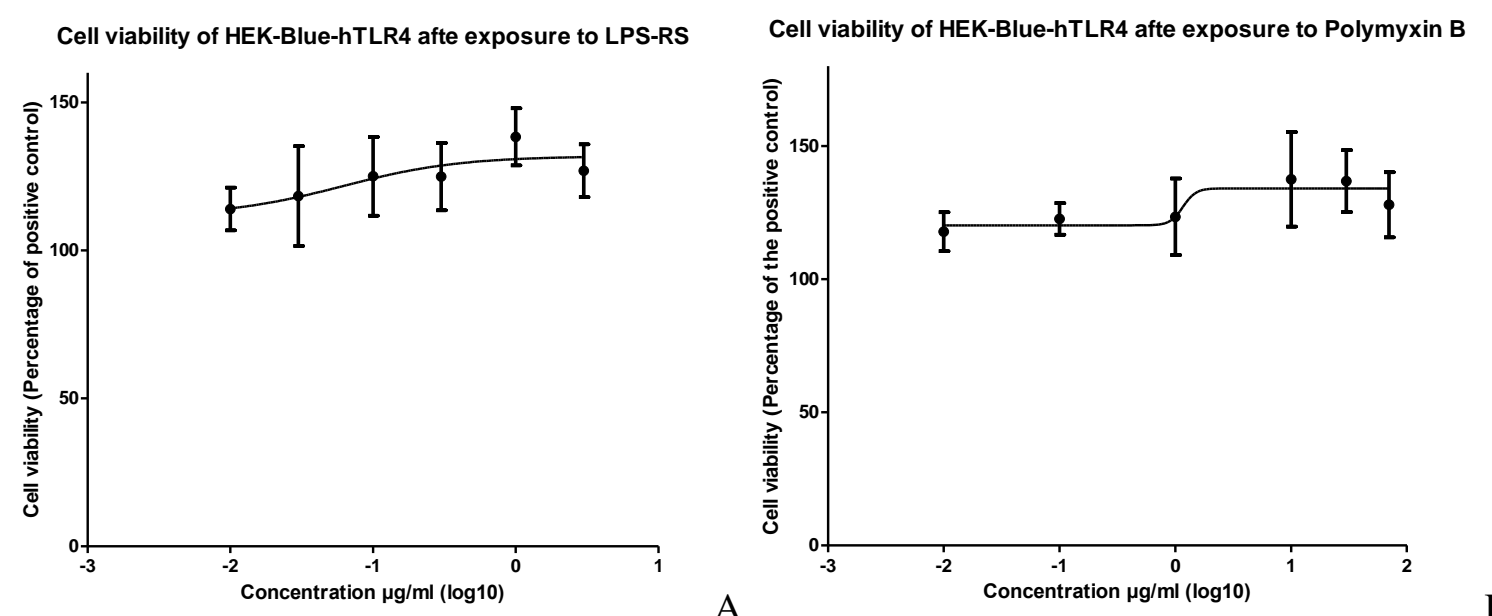

Figure 2 Viability of HEK-Blue-TLR4 cells when exposed to increasing concentrations of LPS-RS (A) and Polymyxin B (B).

\section{Effect of TLR-4 inhibitors on LPS and resistin induced TLR-4 signaling.}

We used two known inhibitors for the determination of the inhibition of LPS induced TLR-4 signaling. The results are shown in figures $3 \mathrm{~A}, 3 \mathrm{~B}$ and $\mathrm{IC}_{50}$ values derived from these graphs are summarized in table 1 . The levels of TLR-4 signaling inhibition by the two compounds are expressed compared to the value at the lowest concentration of inhibitor set to $100 \%$. In figure $2 \mathrm{~A}$ inhibition of LPS by LPS-RS is shown. The $\mathrm{IC}_{50}$ for inhibition of LPS induced TLR-4 gene expression was $0.2 \mu \mathrm{g} / \mathrm{ml}\left(\mathrm{R}^{2}=0.9259\right)$. Polymyxin $\mathrm{B}$ (Figure $2 \mathrm{~b}$ ) inhibited the LPS induced TLR-4 mediated gene expression with an $\mathrm{IC}_{50}$ value of $5.7 \mu \mathrm{g} / \mathrm{ml}\left(\mathrm{R}^{2}=0.99\right)$. Together these data reveal that the TLR- 4 reporter gene assays can be used to detect TRL-4 antagonism towards LPS. 


\section{Chapter 3}
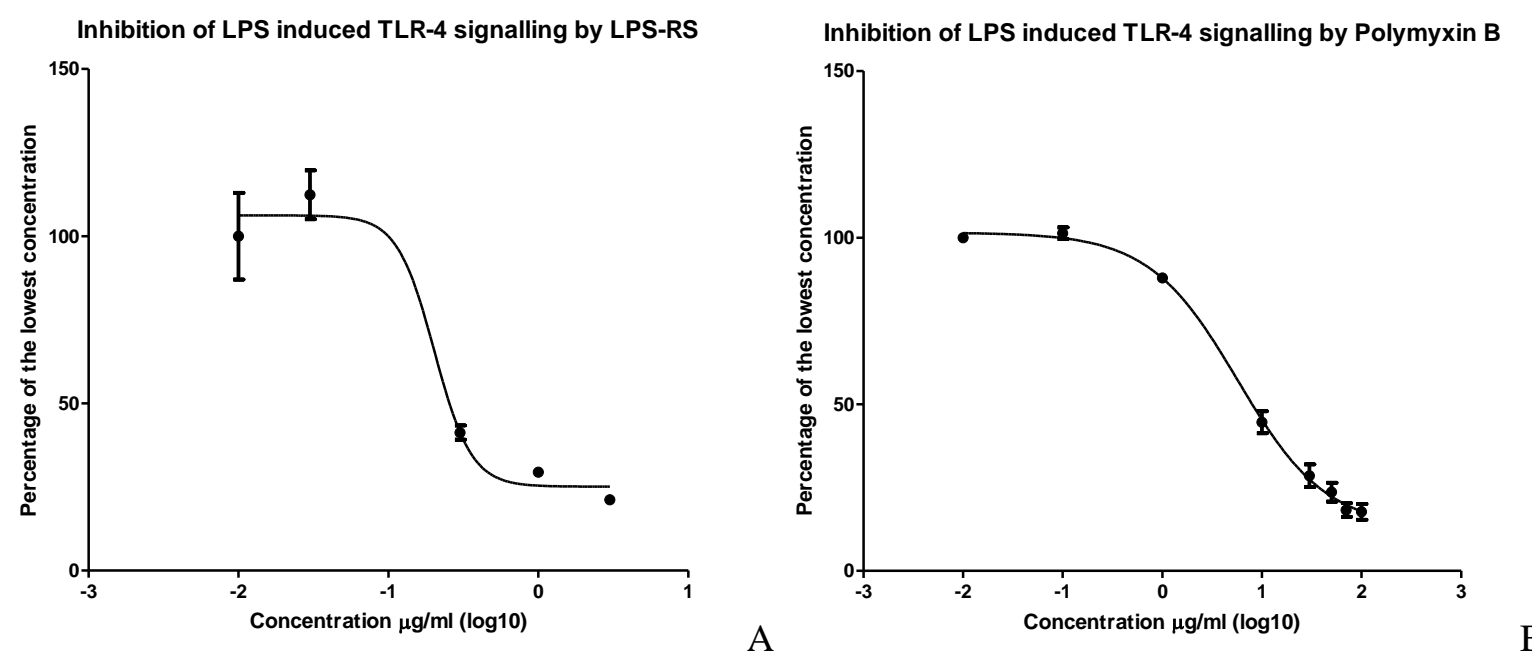

Figure 3. Inhibition of LPS induced TLR-4 signaling by LPS-RS (A) and polymyxin B (B). The concentration of LPS present in the respective incubations amounted to its $\mathrm{EC}_{50}$ value of $0.05 \mu \mathrm{g} / \mathrm{ml}$ for activation as determined from Figure 1A. $\mathrm{IC}_{50}$ values were calculated by Graphpad prism and amounted to $0.2 \mu \mathrm{g} / \mathrm{ml}$ and 5.7 $\mu \mathrm{g} / \mathrm{ml}$ respectively

Figures $4 \mathrm{~A}$ and 4B show the inhibition of the resistin induced TLR-4 mediated gene expression by LPS-RS and Polymyxin B respectively when TLR-4 signaling is induced by resistin. LPS-RS inhibited the resistin-induced TLR-4 signaling with an $\mathrm{IC}_{50}$ of $0.29 \mu \mathrm{g} / \mathrm{ml}$ $\left(\mathrm{R}^{2}=0.7\right)$. Polymyxin $\mathrm{B}$ inhibited the signaling with an $\mathrm{IC}_{50}$ of $7.2 \mu \mathrm{g} / \mathrm{ml}\left(\mathrm{R}^{2}=0.64\right)$. These data reveal that the TRL-4 reporter gene assay could also detect TRL-4 antagonism with respect to resistin as the agonist.
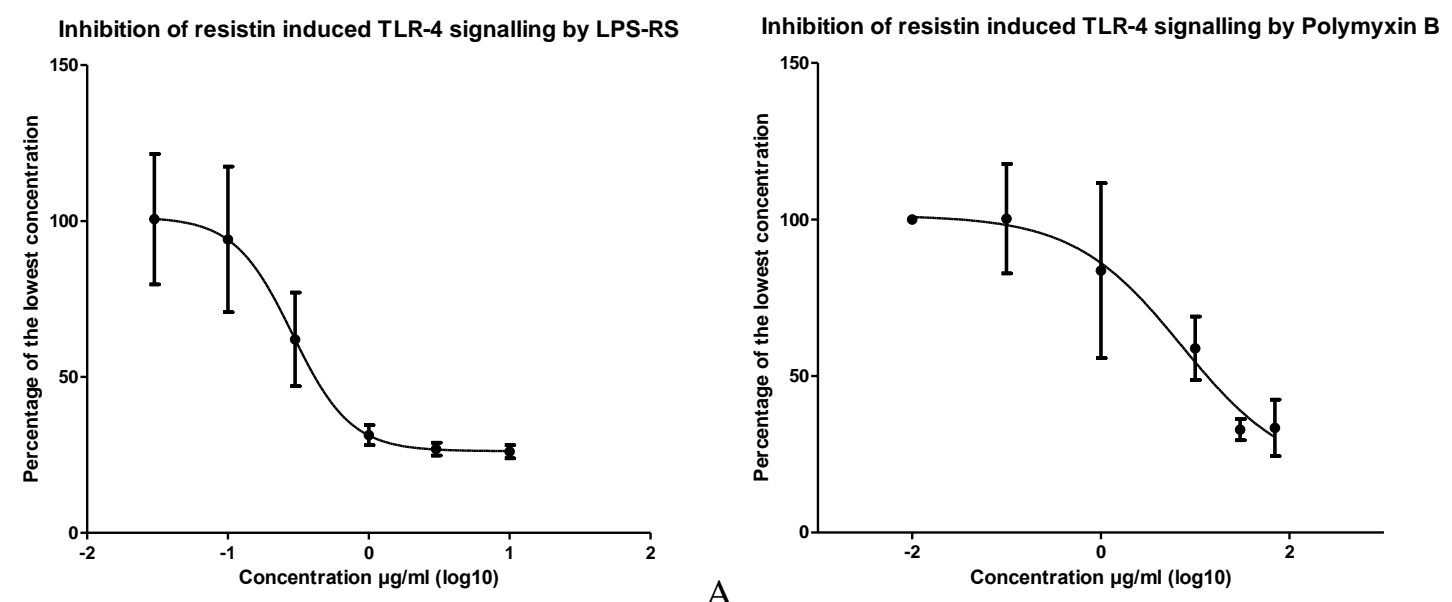

Figure 4. Inhibition of resistin induced TLR-4 signaling by LPS-RS (A) and resistin (B). The concentration of resistin present in the respective incubations amounted to its $\mathrm{EC}_{50}$ value of $5 \mu \mathrm{g} / \mathrm{ml}$ for activation as determined from Figure 1B. $\mathrm{IC}_{50}$ values were calculated by Graphpad prism and amounted to $0.29 \mu \mathrm{g} / \mathrm{ml}$ and $7.2 \mu \mathrm{g} / \mathrm{ml}$ respectively 
Table 1. $\mathrm{IC}_{50}$ values for TLR-4 inhibition by LPS and resistin as determined from the curves presented in figures 3 and 4

\begin{tabular}{|c|c|c|c|c|c|c|}
\hline & \multirow[t]{2}{*}{ LPS } & & \multicolumn{2}{|l|}{ Resistin } & \multicolumn{2}{|c|}{ Literature $\mathrm{IC}_{50}$} \\
\hline & & & & & LPS & Resistin \\
\hline & $\mathrm{IC}_{50}$ & $\mathrm{R}^{2}$ & $\mathrm{IC}_{50}$ & $\mathrm{R}^{2}$ & - & - \\
\hline LPS-RS & $0.2 \mu \mathrm{g} / \mathrm{ml}$ & 0.93 & $0.29 \mu \mathrm{g} / \mathrm{ml}$ & 0.7 & $\begin{array}{l}0.1 \mu \mathrm{g} / \mathrm{ml} \\
\text { (Lu et. al. }\end{array}$ & - \\
\hline Polymyxin B & $5.7 \mu \mathrm{g} / \mathrm{ml}$ & 0.99 & $7.2 \mu \mathrm{g} / \mathrm{ml}$ & 0.64 & $\begin{array}{l}1.6 \mu \mathrm{g} / \mathrm{ml} \\
(\text { Khowniu } \\
2005)\end{array}$ & - \\
\hline
\end{tabular}

\section{Discussion}

The aim of the present study was to investigate whether a reporter gene assay for Toll-like receptor 4 (TLR-4) signaling could be used as a method for detecting resistin antagonism. Given the role of resistin in increasing insulin resistance this may provide a novel target for development of novel drug strategies for T2DM. The connection of resistin with the TLR-4 receptor was made initially by Tarkowski et al. (2010) who reported that resistin competed with LPS for the binding to TLR-4. Taking this into consideration we used a commercially available reporter gene cell line to investigate whether this assay would enable detection of resistin antagonism. Both LPS and resistin were shown to act as agonists able to induce TLR4 mediated gene expression in the reporter gene with $\mathrm{EC}_{50}$ values of $0.05 \mu \mathrm{g} / \mathrm{ml}$ and $5 \mu \mathrm{g} / \mathrm{ml}$ respectively. Stevens et al. (2013) showed a range of $\mathrm{EC}_{50}$ for LPS induced TLR-4 mediated gene expression from 3 experiments of 0.011 to $0.084 \mu \mathrm{g} / \mathrm{ml}$ which complies with our results. As for resistin mediated activation of TLR-4 this study is to the best of our knowledge, the first one reporting an $\mathrm{EC}_{50}$ value for this interaction. In several cell based models with different cell lines (THP-1, PBMC) resistin effect concentrations have been reported, ranging from $50 \mathrm{ng} / \mathrm{ml}$ to $1000 \mathrm{ng} / \mathrm{ml}$ (Tarkowski et al., 2010; Benomar et al., 2012; Lee et al., 2012). Only in one of these studies an $\mathrm{EC}_{50}$ for a resistin induced effect was presented representing an $\mathrm{EC}_{50}$ for induction of growth hormone release, but not for TLR-4. $\mathrm{EC}_{50}$ s were obtained for dispersed anterior pituitary cells exposed to resistin (Rodríguez-Pacheco et. al., 2009) for 4 and 24 hours and amounted to $3.5 \mathrm{nM}$ and $2.6 \mathrm{pM}$ respectively. Normally serum resistin in humans ranges from 7 to $22 \mathrm{ng} / \mathrm{ml}$ but is significantly increased in patients with T2DM (Jamaluddin et al., 2011; Steppan et al., 2001). It is also increased in patients with metabolic syndrome and its related diseases such as coronary artery disease and cardiovascular disease (Ohmori et al., 2005; Reilly et al., 2005). In a study with patients with acute coronary syndrome serum resistin levels reported as high as $1.18 \mu \mathrm{g} / \mathrm{ml}$ (Wang et al., 2009). Nonetheless there are differences in the quantitative observations reported by different groups 


\section{Chapter 3}

a phenomenon most probably reflecting resistin's secretion timing (McTernan et al., 2003; Jamaluddin et al., 2011).

In a next step the inhibition of the LPS induced TLR-4 mediated gene expression by selected inhibitors was characterized. For LPS-RS it has been reported that it inhibits LPS mediated effects by competing with LPS for the same binding site on MD-2 (Golenblock et al., 1991; Coats et al., 2005). Polymyxin b has been reported as an endotoxin binding protein able to inhibit LPS mediated effects by binding to lipid A, the toxic component of LPS (Duff et al., 1982; Shoenfelt et al., 2009; Birknmeier et al., 2006; Bhor et al., 2005). The results obtained in the present study revealed that both inhibitors were able to reduce the LPS mediated TLR-4 gene expression in the reporter gene assay with $\mathrm{IC}_{50}$ values of $0.2 \mu \mathrm{g} / \mathrm{ml}$ and $5.73 \mu \mathrm{g} / \mathrm{ml}$ for LPS-RS and polymixin $b$ respectively. $\mathrm{Lu}$ et al. reported an $\mathrm{IC}_{50}$ of LPS-RS for LPS inhibition at $0.1 \mu \mathrm{g} / \mathrm{ml}$ which is close to our results. Khownium et al. (2005) showed an $\mathrm{IC}_{50}$ for polymyxin B inhibition of LPS induced TLR-4 mediated effects of $1.6 \mu \mathrm{g} / \mathrm{ml}$.

In a next step we tested if these inhibitors would also inhibit resistin induced TLR-4 signaling. Both compounds showed resistin antagonism, inhibiting resistin mediated TLR-4 induced gene expression. Comparison of the $\mathrm{IC}_{50} \mathrm{~S}$ for inhibition of the LPS and resistin induced TLR4 signaling reveals that the $\mathrm{IC}_{50}$ s for LPS-RS and also for Polymyxin $\mathrm{B}$ are quite close differing less than 2-fold.

Based on the results obtained it can be concluded that the HEK-Blue-hTLR4 cell line which is commercially available can be used to screen for compounds that may inhibit resistin induced TLR-4 signaling, reflecting resistin antagonism. Given the role of resistin in increasing insulin resistance in T2DM (Benomar et al., 2012; Benomar et al., 2016) this assay may proof of value as a novel target in high throughput screening of compounds that could counteract insulin resistance via resistin antagonism. Whether this will eventually lead to novel classes of drugs for T2DM remains to be established.

\section{Acknowledgement}

This research was financially supported by the Dutch Ministry of Economic Affairs, Agriculture and Innovation, Grant Pieken in de Delta - Healthy ageing project, PID082022. 


\section{References}

Barnes, K. M. and J. L. Miner (2009). "Role of resistin in insulin sensitivity in rodents and humans." Curr Protein Pept Sci 10(1): 96-107.

Benomar, Y., et al. (2016). "Central Resistin/TLR4 Impairs Adiponectin Signaling, Contributing to Insulin and FGF21 Resistance." Diabetes 65(4): 913-926.

Benomar, Y., et al. (2013). "Central Resistin Overexposure Induces Insulin Resistance Through Toll-Like Receptor 4." Diabetes 62(1): 102-114.

Bhor, V. M., et al. (2005). "Polymyxin B: An ode to an old antidote for endotoxic shock." Molecular Biosystems 1(3): 213-222.

Birkenmeier, G., et al. (2006). "Polymyxin B-conjugated alpha 2-macroglobulin as an adjunctive therapy to sepsis: Modes of action and impact on lethality." Journal of Pharmacology and Experimental Therapeutics 318(2): 762-771.

Boden, G. (1997). "Role of fatty acids in the pathogenesis of insulin resistance and NIDDM." Diabetes 46(1): 3-10.

Coats, S. R., et al. (2005). "MD-2 mediates the ability of tetra-acylated and penta-acylated lipopolysaccharides to antagonize Escherichia coli lipopolysaccharide at the TLR4 signaling complex." J Immunol 175(7): 4490-4498.

Daquinag, A. C., et al. (2011). "An isoform of decorin is a resistin receptor on the surface of adipose progenitor cells." Cell Stem Cell 9(1): 74-86.

Duff, G. W. and E. Atkins (1982). "The Inhibitory Effect of Polymyxin-B on EndotoxinInduced Endogenous Pyrogen Production." J Immunol Methods 52(3): 333-340.

Golenbock, D. T., et al. (1991). "Lipid-a-Like Molecules That Antagonize the Effects of Endotoxins on Human Monocytes." Journal of Biological Chemistry 266(29): 1949019498.

Haugen, F., et al. (2001). "Inhibition by insulin of resistin gene expression in 3T3-L1 adipocytes." FEBS Lett 507(1): 105-108.

Hoshino, K., et al. (1999). "Cutting edge: Toll-like receptor 4 (TLR4)-deficient mice are hyporesponsive to lipopolysaccharide: evidence for TLR4 as the Lps gene product." $\mathrm{J}$ Immunol 162(7): 3749-3752.

Kawai, T. and S. Akira (2006). "TLR signaling." Cell Death Differ 13(5): 816-825.

Kawamoto, T., et al. (2008). "TAK-242 selectively suppresses Toll-like receptor 4-signaling mediated by the intracellular domain." Eur J Pharmacol 584(1): 40-48.

Khownium, K., et al. (2006). "Novel endotoxin-sequestering compounds with terephthalaldehyde-bis-guanylhydrazone scaffolds." Bioorg Med Chem Lett 16(5): 13051308.

Lazar, M. A. (2007). "Resistin- and Obesity-associated metabolic diseases." Horm Metab Res 39(10): 710-716. 


\section{Chapter 3}

Lee, E. S., et al. (2012). "Caffeic Acid Disturbs Monocyte Adhesion onto Cultured Endothelial Cells Stimulated by Adipokine Resistin." J Agric Food Chem 60(10): 27302739.

Lu, Z. Y., et al. (2013). "TLR4 antagonist reduces early-stage atherosclerosis in diabetic apolipoprotein E-deficient mice." Journal of Endocrinology 216(1): 61-71.

McTernan, P. G., et al. (2003). "Resistin and type 2 diabetes: regulation of resistin expression by insulin and rosiglitazone and the effects of recombinant resistin on lipid and glucose metabolism in human differentiated adipocytes." J Clin Endocrinol Metab 88(12): 60986106.

Medzhitov, R. (2001). "Toll-like receptors and innate immunity." Nature Reviews Immunology 1(2): 135-145.

Nagaev, I., et al. (2006). "Human Resistin Is a Systemic Immune-Derived Proinflammatory Cytokine Targeting both Leukocytes and Adipocytes." PLoS One 1(1).

Ohmori, R., et al. (2005). "Associations between serum resistin levels and insulin resistance, inflammation, and coronary artery disease." J Am Coll Cardiol 46(2): 379-380.

Owecki, M., et al. (2011). "Serum Resistin Concentrations are Higher in Human Obesity but Independent from Insulin Resistance." Experimental and Clinical Endocrinology \& Diabetes 119(2): 117-121.

Patel, S. D., et al. (2004). "Disulfide-dependent multimeric assembly of resistin family hormones." Science 304(5674): 1154-1158.

Poltorak, A., et al. (1998). "Defective LPS signaling in C3H/HeJ and C57BL/10ScCr mice: Mutations in Tlr4 gene." Science 282(5396): 2085-2088.

Pradhan, A. D., et al. (2001). "C-reactive protein, interleukin 6, and risk of developing type 2 diabetes mellitus." Jama-Journal of the American Medical Association 286(3): 327-334.

Qureshi, S. T., et al. (1999). "The Lps locus: genetic regulation of host responses to bacterial lipopolysaccharide." Inflamm Res 48(12): 613-620.

Reilly, M. P., et al. (2005). "Resistin is an inflammatory marker of atherosclerosis in humans." Circulation 111(7): 932-939.

Rodriguez-Pacheco, F., et al. (2009). "Resistin Regulates Pituitary Somatotrope Cell Function through the Activation of Multiple Signaling Pathways." Endocrinology 150(10): 46434652 .

Sanchez-Solana, B., et al. (2012). "Mouse Resistin Modulates Adipogenesis and Glucose Uptake in 3T3-L1 Preadipocytes Through the ROR1 Receptor." Molecular Endocrinology 26(1): 110-127.

Schwartz, D. R. and M. A. Lazar (2011). "Human resistin: found in translation from mouse to man." Trends in Endocrinology and Metabolism 22(7): 259-265. 
Shoenfelt, J., et al. (2009). "Involvement of TLR2 and TLR4 in inflammatory immune responses induced by fine and coarse ambient air particulate matter." Journal of Leukocyte Biology 86(2): 303-312.

Spranger, J., et al. (2003). "Inflammatory cytokines and the risk to develop type 2 diabetes Results of the prospective population-based European Prospective Investigation into Cancer and Nutrition (EPIC)-Potsdam study." Diabetes 52(3): 812-817.

Steppan, C. M., et al. (2001). "The hormone resistin links obesity to diabetes." Nature 409(6818): 307-312.

Stevens, C. W., et al. (2013). "Pharmacological characterization of LPS and opioid interactions at the toll-like receptor 4." British Journal of Pharmacology 168(6): 14211429.

Tarkowski, A., et al. (2010). "Resistin competes with lipopolysaccharide for binding to tolllike receptor 4." J Cell Mol Med 14(6B): 1419-1431.

Wang, H., et al. (2009). "High serum resistin level may be an indicator of the severity of coronary disease in acute coronary syndrome." Chin Med Sci J 24(3): 161-166. 



\title{
Chapter 4
}

\section{Resistin exposure of human omental}

adipocytes affects transcriptional regulation processes involved in cellular differentiation and maintenance.

\author{
A. Sotiriou \\ L. Gijsbers \\ S. Denissov \\ I.M.C.M. Rietjens \\ M. Musters \\ J. Vervoort
}




\title{
Chapter 4
}

\begin{abstract}
Resistin is a small cysteine-rich protein hormone involved in inflammation and insulin resistance. Resistin is mainly secreted from adipose tissue in mice and from immune and epithelial cells but also from adipocytes in humans. An increase in obesity and insulin resistance was observed to be correlated with increased levels of resistin. The precise mode of action of resistin is however still unclear. To better understand the cellular processes that are altered by resistin, we profiled human omental adipocytes, with and without resistin treatment, by means of next generation sequencing (RNA-sequencing) to determine the resistin specific modulation of gene expression. In total, 55 genes were significantly up or down regulated under the influence of resistin in human omental adipocytes. Pathway analysis with Ingenuity Pathway Analysis showed basically 2 networks that are affected by the identified genes. These two networks are "protein trafficking, cellular assembly and organization and cellular function and maintenance" and "cell death and survival, cancer and organismal injury and abnormalities". An analysis of the specific function of the genes up and down regulated with regards to the two networks mentioned indicate that resistin is involved in modifications in transcriptional regulation processes with a role in cellular differentiation and maintenance processes. From the gene expression data it can be proposed that resistin is a survival regulator and is involved in lipid storage. Resistin appears to be expressed when energy conservation is essential. When energy conservation is accompanied by food overconsumption, obesity, inflammation and insulin resitance can be the consequence. Resistin can therefore be considered as a biomarker protein for these health related issues.
\end{abstract}




\section{Introduction}

In 2014, the WHO, estimated the number of diabetic people at 422 million, compared to 108 million in 1980. Obesity is one of the main factors contributing to type 2 diabetes mellitus (T2DM), but it is also linked to insulin resistance, cardiovascular diseases and cancer (Benomar et al., 2012, Caballero, 2003; Calle et al., 2003). In 2014, 1.9 billion adults were overweight and 600 million were obese (WHO, 2015; Cabia et al., 2016). Obesity is characterized by the excessively storage of body fat in adipose tissue, leading to a chronic inflammatory state (Sousa-Santos et. al., 2013; Lumeng and Saltiel, 2011; Freitas-Lima et. al., 2015). Adipose tissue operates as an endocrine organ (Cabia et al., 2016) that secretes adipocytokines such as leptin, adiponectin, resistin and IL-6 (Wang et. al., 2004; Galic et al., 2010). These adipocytokines play a major role in energy homeostasis and metabolism. In obese persons, changes occur in the secretion of these inflammatory cytokines, which can affect insulin sensitivity through effects on the energy homeostasis (Kahn and Flier, 2000; Wellen and Hotamisligil, 2003; Steppan et al., 2001; Olefsky and Class, 2009).

Resistin is a $12.5 \mathrm{kDa}$ hormone that is mainly secreted by adipocytes in mice, while in humans immune and epithelial cells but also adipocytes can secrete resistin (McTernan et al., 2002; Cabia et a., 2016). The expression in human adipose tissue is suggested to originate from resident inflammatory cells (Schwartz and Lazar, 2011; Patel et al., 2003; McTernan et al., 2002), but adipocytes can also contribute to resistin expression (McTernan et al., 2002; Benomar et al., 2012; Reilly et al., 2005; Silha et al., 2003; Vendrell et al. 2004). Resistin has 6 disulfide bonds, which suggests that the protein is very stable in an in vivo situation as degradation by proteases will be hampered by the compact global inert structure (Patel et al., 2004).

In this study we investigated the mechanism behind resistin mediated effects by exposing human adipocytes to resistin and analyzing its effect on gene expression. We identified genes that were up or down regulated by resistin in human adipocytes using RNA sequencing technology (RNAseq).

\section{Materials and methods}

\section{Human omental adipocytes}

Human omental adipocytes (Omental Adipocytes 96-Well PlateSuperlot, mixed donor lot)(Zen-bio, NC, USA) were used for this study. The cells were from a female non-diabetic, 


\section{Chapter 4}

non-smoking individual with BMI of 45.84 and age 34.6. Those cells were already differentiated and delivered in a multiwell plate, so no (sub)culturing had to be done. Every 34 days the medium was refreshed with Omental Basal Medium (OM-BM) (Tebu-bio, Netherlands) supplemented with $1 \mathrm{x}$ penstrep.

\section{Exposure of cells to resistin and RNAseq sampling}

Regarding the RNAseq sampling cells were treated as follows. Human omental adipocytes were maintained according to manufacturer's protocol. Medium was removed from the wells and the cells were washed one time with Phosphate Buffered Saline (PBS)(Gibco, Paisley, Scotland), Netherlands). OM-BM was added containing either $1 \mu \mathrm{M}$ human resistin (SigmaAldrich, St. MO, USA) or no resistin (control). Cells were treated for 18 hours and subsequently harvested in Qiazol (Qiagen, Venlo, NL).

\section{rRNA removal with ribo-zero removal kit.}

In more detail, magnetic beads had to be washed before using them. Therefore magnetic beads were added to an eppendorf tube and washed, pelleted, re-suspended in storage buffer and until used in room temperature and the tube was placed on a magnetic stand for $1 \mathrm{~min}$. Remaining beads were stored at $2{ }^{\circ} \mathrm{C}$ to $8{ }^{\circ} \mathrm{C}$. In a separate eppendorf tube total RNA isolated from the adipocytes was treated with rRNA removal solution provided by the manufacturer. Treated sample was incubated and rRNA probes hybridize to rRNA.

\section{Ribo-zero removal kit - preparation of magnetic beads.}

For each sample, $225 \mu \mathrm{l}$ of the Magnetic Beads were transferred into an RNase-free tube slowly avoiding bubble formation. Tubes were placed on a magnetic stand for 1 min until the solutions became clear. Then supernatant was removed. Tubes were removed from the magnetic stand, and $225 \mu \mathrm{l}$ of RNase-Free Water were added after which the magnetic beads were resuspended by vortexing for 3 times. Tubes were then replaced on the magnetic stand for $1 \mathrm{~min}$ until the solutions became clear again after which the remaining water was discarded. Next $65 \mu$ of Magnetic Bead Resuspension Solution were added and the beads were resuspended by vortexing once.

\section{Ribo-zero rRNA removal kit - preparation of samples.}

In an RNase-free microcentrifuge tube $20 \mu \mathrm{l}$ of total RNA, $8 \mu \mathrm{l}$ of RNase-free water, $8 \mu \mathrm{l}$ of rRNA removal solution (containing probes that hybridizes to rRNA) and $4 \mu$ of rRNA 
reaction buffer were added. The solution was mixed thoroughly and incubated for $10 \mathrm{~min}$ at $68{ }^{\circ} \mathrm{C}$. Any condensation left was removed and the solution was incubated at room temperature for $5 \mathrm{~min}$.

For each sample $40 \mu \mathrm{l}$ of probe-hybridized RNA sample were transferred to the $65 \mu 1$ of washed, room temperature Magnetic Beads. Immediately and thoroughly the contents were mixed by pipetting 10-15 times. Additionally tubes were vortexed at high speed for a minimum of $10 \mathrm{sec}$ and incubated for $5 \mathrm{~min}$ at room temperature. After this, the tubes were incubated at $50{ }^{\circ} \mathrm{C}$ for 5 minutes. After this incubation the tubes were placed on a magnetic stand for at least 1 minute until the solutions became clear. The supernatant containing the sample depleted of rRNA was removed, and transferred to an appropriately sized RNase-free microcentrifuge tube in $-20{ }^{\circ} \mathrm{C}$.

\section{Ethanol precipitation of the rRNA-depleted sample.}

The volume of each rRNA-depleted sample was adjusted to $180 \mu 1$ using RNase-Free Water. $18 \mu \mathrm{l}$ of $3 \mathrm{M}$ sodium acetate and $2 \mu \mathrm{l}$ of glycogen $(10 \mathrm{mg} / \mathrm{ml})$ were added to each tube, followed by gentle vortexing. Additionally 3 volumes $(600 \mu \mathrm{l})$ of ice-cold $100 \%$ ethanol were added and the samples were vortexed gently. Tubes were placed at $-20{ }^{\circ} \mathrm{C}$ for at least 1 hour. Subsequently the samples were centrifuged at $10,000 \mathrm{x}$ g for 30 minutes and the supernatants were carefully removed and discarded. The pellets were washed with ice-cold $70 \%$ ethanol and centrifuged at higher than $10,000 \times \mathrm{g}$ for 5 minutes. The supernatants were removed and discarded. This washing step with ethanol was repeated twice with $70 \%$ ethanol. Centrifugation was repeated to collect and remove any residual supernatant. The final pellets containing rRNA-depleted RNA were air dried at room temperature for 5 min. The rRNAdepleted RNA pellets were dissolved in $10 \mu \mathrm{l}$ of RNase-Free Water and samples were stored in $-20{ }^{\circ} \mathrm{C}$ overnight.

\section{ScriptSeq RNA-seq library preparation kit - cDNA synthesis}

For the fragmentation of RNA we used in a total of $12 \mu 1,1 \mu 1$ fragmentation solution, $2 \mu 1$ cDNA Primer and $9 \mu \mathrm{l}$ of dissolved rRNA-depleted RNA incubated for 5 min at $85{ }^{\circ} \mathrm{C}$ in a thermocycler with heated lid. RNA fragmentation was stopped by placing the sample on ice. The next step was the synthesis of cDNA. To this end to each sample were added $4 \mu \mathrm{l}$ of cDNA synthesis master mix ( $3 \mu \mathrm{l}$ of cDNA synthesis, $0.5 \mu 1$ of $100 \mathrm{mM}$ DTT and $0.5 \mu \mathrm{l}$ of StarScript AMV Reverse Transcriptase). Incubation at $25^{\circ} \mathrm{C}$ for $5 \mathrm{~min}$ was followed by 


\section{Chapter 4}

incubations at $42{ }^{\circ} \mathrm{C}$ for $20 \mathrm{~min}$. Then the reactions were cooled to $37{ }^{\circ} \mathrm{C}$. One by one the reactions were taken out from the thermocycler and $1 \mu$ of Finishing Solution was added. Incubation at $37{ }^{\circ} \mathrm{C}$ for 10 minutes followed. After this incubation, each sample was incubated at $95{ }^{\circ} \mathrm{C}$ for $3 \mathrm{~min}$ and finally cooled to $25{ }^{\circ} \mathrm{C}$. For the tagging of DNA $8 \mu \mathrm{l}$ of Terminal Tagging Master Mix (7.5 $\mu \mathrm{l}$ of Terminal Tagging Premix and $0.5 \mu \mathrm{l}$ of DNA Polymerase) were added to each sample followed by thorough mixing and incubation first at $25{ }^{\circ} \mathrm{C}$ for $15 \mathrm{~min}$ and then at $95{ }^{\circ} \mathrm{C}$ for $3 \mathrm{~min}$. Finally the reactions were cooled to $4{ }^{\circ} \mathrm{C}$.

For PCR amplification the cDNA was purified. To this end the MinElute PCR purification Kit (Qiagen, Venlo, NL) was used. According to the manufacturer 5 volumes of Buffer PB were added to 1 volume of the PCR reaction and mixed thoroughly. MinElute columns were placed in collection tubes provided by the manufacturer. Samples were applied to the MinElute columns and centrifuged for $1 \mathrm{~min}$. The flow-through was discarded. For the washing, $750 \mu \mathrm{l}$ of PE buffer were put to the columns and centrifuged for $1 \mathrm{~min}$. To completely remove ethanol from PE buffer the columns were additionally centrifuged for $1 \mathrm{~min}$ at maximum speed. Afterwards columns were put in clean tubes. To elute DNA, $10 \mu \mathrm{EB}$ buffer were added in the center of the membrane, let for $1 \mathrm{~min}$ and the samples were centrifuged for 1 $\min$.

The samples were sent to ServiceXS (Leiden, Netherlands) and sequenced with an Illumina Hi-Seq instrument. Single $50 \mathrm{nt}$ reads were aligned to hg19 genome assembly by TopHat and analysed by SAMtools and BAMtools. The analysis of the RNA-seq was processed in R/Bioconductor with the program DESeq (standard settings) to identify the differentially expressed genes, as well as generating the volcano plot.

\section{Pathway analysis with Ingenuity Pathway Analysis software}

The results of RNAseq gene expression data expressed as a ratio of resistin exposed adipocytes versus unexposed adipocytes along with the gene IDs with the concomitant pvalues were uploaded to Ingenuity Pathway Analysis (IPA). In IPA the information uploaded is matched to databases (based on an indepth analysis of biological literature). IPA generates algorithmically biological networks and defines canonical pathways and functions related to the genes uploaded. Each network acquires a score that indicates the possibility that the identified genes within the network are clustered together with focus genes that are from the uploaded list, pass filters that are applied, and are available for generating networks (http://www.ingenuity.com). 


\section{Results}

The exposure of adipocytes to resistin modulated significantly the expression of 55 genes. In table 1 the genes that were significantly differentially expressed after resistin exposure with the respective $\log 2$ fold change and p-value are shown. The $\log 2$ difference shows the effect of the presence as compared to the absence of resistin, while the p-value of 0.05 was applied to rule out effects that are purely random.

Table 1. Significantly up or down regulated genes after exposure of human adipocytes to resistin followed by the p-value. Inf stands for infinite $\log 2$ change indicating the absence of the gene in one of the two samples.

\begin{tabular}{|c|c|c|c|c|c|}
\hline Upregulated & p-value & Log2 change & Downregulated & p-value & Log2 change \\
\hline FLJ45340 & $7.43 e-21$ & 7.199672 & SNRPD2 & $3.44 \mathrm{e}-14$ & -3.05784 \\
\hline SIX4 & $9.36 \mathrm{e}-12$ & 5.178914 & KCTD7 & $2.07 e-13$ & -6.28716 \\
\hline ZNF138 & $6.16 \mathrm{e}-11$ & 6.894818 & HYAL3 & $1.28 \mathrm{e}-12$ & -6.18133 \\
\hline UFM-1 & $4.37 \mathrm{e}-11$ & 6.083479 & SETD4 & $9.35 \mathrm{e}-09$ & -Inf \\
\hline CNTFR & $3.64 \mathrm{e}-08$ & 4.988685 & LOC100133161 & $5.99 \mathrm{e}-08$ & $-\operatorname{Inf}$ \\
\hline C11orf10 & $1.84 \mathrm{e}-08$ & 3.269259 & DQ596074 & $5.88 \mathrm{e}-07$ & -Inf \\
\hline DQ598070 & $8.01 \mathrm{e}-07$ & Inf & DQ592203 & $3.37 \mathrm{e}-07$ & -Inf \\
\hline ISY1 & $4.34 \mathrm{e}-07$ & $\operatorname{Inf}$ & SNORD25 & $3.08 \mathrm{e}-07$ & -Inf \\
\hline AK023614 & $1.94 \mathrm{e}-07$ & Inf & JA429802 & 8.37e-06 & -1.954 \\
\hline C19orf42 & $7.89 \mathrm{e}-06$ & $\operatorname{Inf}$ & CHURC1-FNTB & $6.91 \mathrm{e}-06$ & -Inf \\
\hline MIR4731 & $3.41 \mathrm{e}-06$ & Inf & C4orf43 & $3.81 \mathrm{e}-06$ & -4.28011 \\
\hline SNORD113-1 & $3.41 \mathrm{e}-06$ & Inf & CNP & $1.73 \mathrm{e}-06$ & -3.63176 \\
\hline MIR4800 & $1.21 \mathrm{e}-06$ & Inf & DQ595216 & $9.89 \mathrm{e}-05$ & $-\operatorname{Inf}$ \\
\hline LPL & $7.89 \mathrm{e}-05$ & 2.134426 & U42379 & $7.32 \mathrm{e}-05$ & $-\operatorname{Inf}$ \\
\hline SNORD114-9 & $7.89 \mathrm{e}-05$ & 2.134426 & DQ786325 & $6.69 \mathrm{e}-05$ & -3.67646 \\
\hline MIR611 & $5.42 \mathrm{e}-05$ & $\operatorname{Inf}$ & ZFP161 & $4.75 \mathrm{e}-05$ & -3.84472 \\
\hline MIR125B1 & $5.15 \mathrm{e}-05$ & 3.839776 & C15orf38 & $1.12 \mathrm{e}-05$ & -Inf \\
\hline IFIT5 & $4.30 \mathrm{e}-05$ & 2.138414 & DQ576933 & 0.000302 & -Inf \\
\hline DM074112 & $2.83 e-05$ & Inf & DQ588370 & 0.000302 & -Inf \\
\hline FU270179 & $2.83 e-05$ & Inf & JA535805 & 0.000181 & -Inf \\
\hline FU270191 & $2.83 e-05$ & $\operatorname{Inf}$ & ZNF846 & 0.000109 & -Inf \\
\hline TIMM8A & $2.83 e-05$ & Inf & FLJ27352 & 0.000125 & -2.54785 \\
\hline MIR1206 & $1.49 \mathrm{e}-05$ & $\operatorname{Inf}$ & & & \\
\hline DQ595173 & 0.000313 & Inf & & & \\
\hline MIR181A1 & 0.000313 & $\operatorname{Inf}$ & & & \\
\hline SNORD115-5 & 0.000313 & Inf & & & \\
\hline AF047486 & 0.000263 & 2.383704 & & & \\
\hline DQ596932 & 0.000251 & Inf & & & \\
\hline DQ598479 & 0.000251 & Inf & & & \\
\hline CPEB3_ribozyme & 0.000201 & Inf & & & \\
\hline SNORD1A & 0.000166 & 2.222392 & & & \\
\hline DQ574699 & 0.00016 & 2.818582 & & & \\
\hline SNORD114-3 & 0.000129 & 2.971331 & & & \\
\hline
\end{tabular}

FLJ45340 showed the most significant upregulation with $7.199 \log 2$ fold change with a pvalue of 7.43e-21. SIX4, ZNF138, UFM1, CNTFR, C11orf10 DQ59870 and ISY1 followed. 


\section{Chapter 4}

Among the genes that were significantly upregulated were also a number of micro RNAs (miRNAs) (MIR4800, MIR4731, MIR2016, MIR611, MIR181A1, MIR125B1), small nucleolar RNAs (snoRNA) (SNORD113-1, SNORD115-5, SNORD114-3, SNORD1A, SNORD114-9) and piwi-RNAs (DQ598070, DQ595173, DQ596932, DQ598479, DQ574699).

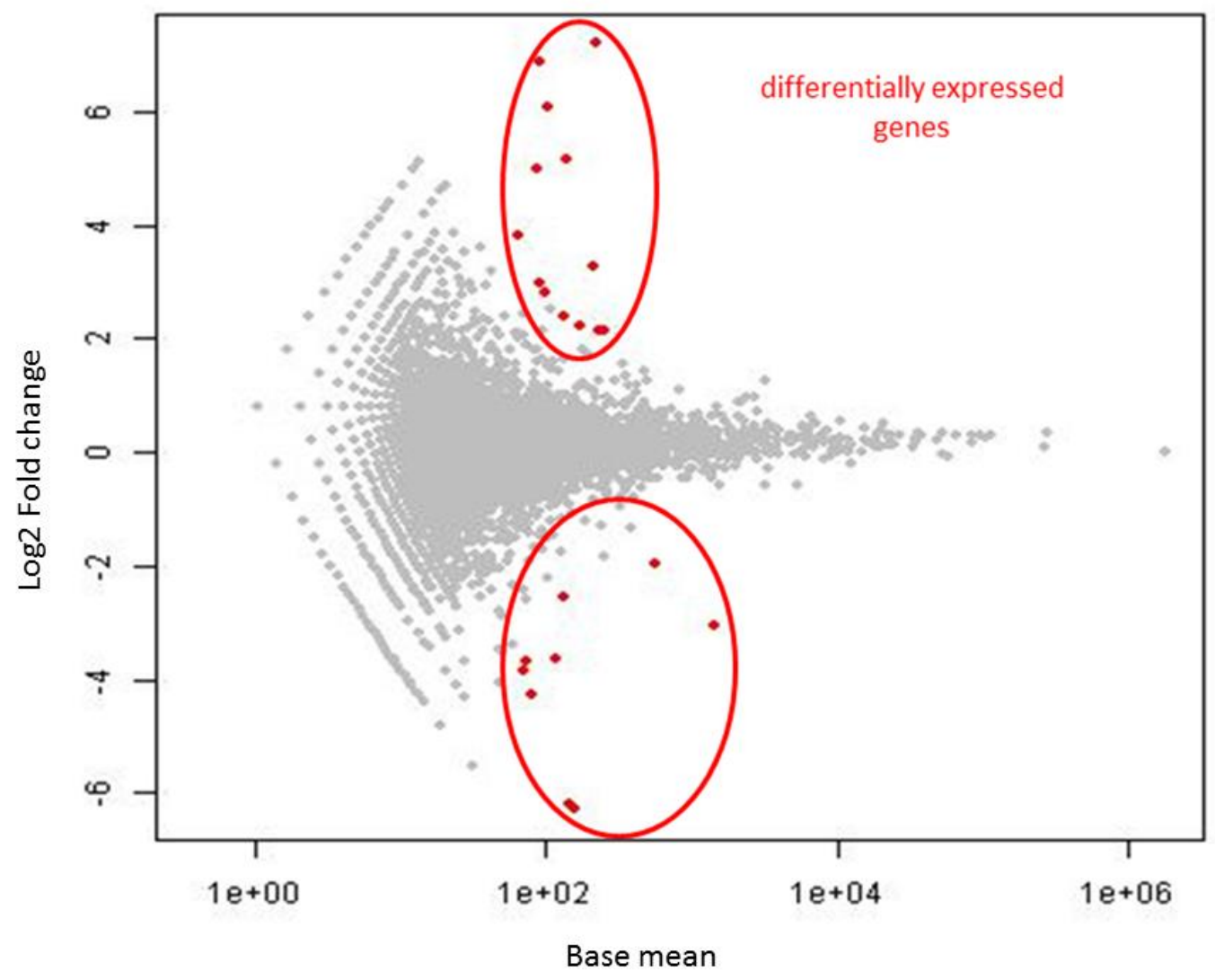

Figure 1. Volcano plot showing the $\log 2$ fold change of the genes identified versus the $\log 2$ significance of the change. The red color dots represent the genes that changed significantly $(\mathrm{p}<0.05)$ (see Table 1$)$. Only genes present in both samples are represented, so genes absent in one sample of the two samples are not shown.

The gene that showed the most significant downregulation was SNRPD2 with a log 2 fold change of -3.057 and a p-value of 3.44e-14. KCTD7, HYAL3 and SETD4 followed. Only one small nucleolar RNAs (SNORD25) and several piwi-RNAs (DQ596074, DQ592203, DQ595216, DQ786325, DQ576933, DQ588370) were found to be significantly downregulated.

Figure 2 shows the localization of the proteins encoded by the genes according to Uniprot (www.uniprot.org). SIX4, ZNF138, UFM-1, ISY1, CPEB3_ribozyme, SNRPD2, ZFP161, ZNF846 are positioned in the nucleus. UFM-1, CPEB3_ribozyme, SNRDP2, HYAL3, CNP, KCTD7 are positioned in the cytoplasm. CNTFR, C11ord10, C19orf42, LPL, IFIT5, KCTD7, 
CNP, DQ786325 are positioned in the membrane. TIMM8A, CNP, DQ786325 (MTX1) are positioned in the mitochondrion. C15orf38 is positioned in the lamellipodium. SETD4 is proposed to be either in the nucleus or in the ribosome. There is little information about the positioning of FLJ45340, DQ598070, LOC100133161, AK023614, DQ596074, DM074112, DQ592203, FU270179, JA429802, FU270191, CHURC1-FNTB, DQ595173, C4orf43, AF047486, DQ595216, DQ596932, U42379, DQ598479, DQ576933, DQ574699, DQ588370 and JA535805.

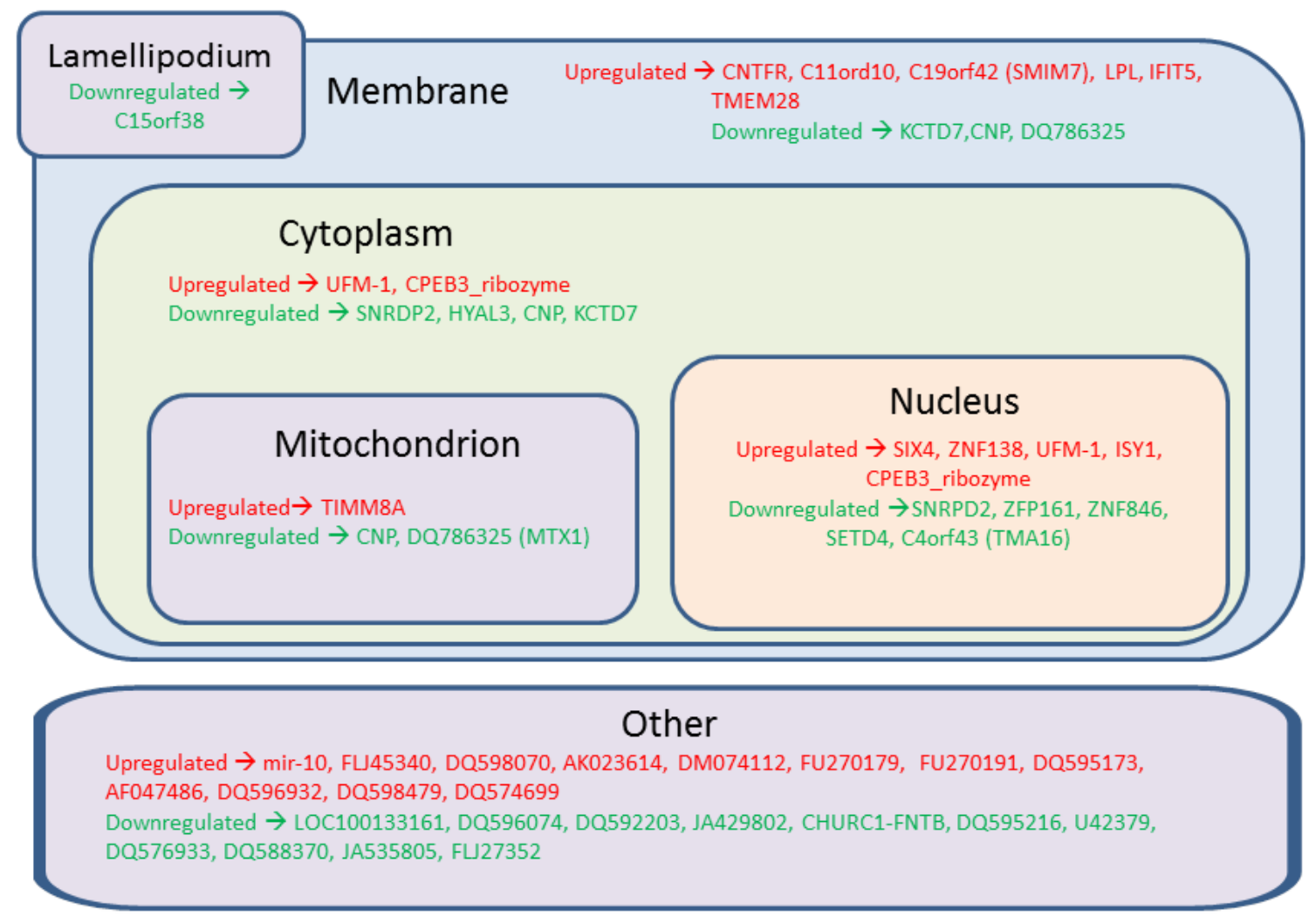

Figure 2. Position of genes in the cell that are significantly $(\mathrm{p}<0.05)$ up or down regulated after exposure of human adipocytes to resistin.

The 55 genes that are significantly differentially expressed when cells are exposed to resistin were uploaded to IPA software for further pathway analysis. Table 2 shows the two IPA networks which were shown to be important when analyzing the role of the differentially expressed genes, and the genes that are involved in these networks. More specifically network 1, which included top diseases and functions such as protein trafficking, assembly and organization, cellular function and maintenance, scored 29 with 12 focus genes that were actually differentially expressed of all genes linked to this pathway. Network 2, which included top diseases and functions such as cell death and survival, cancer, organismal injury and abnormalities, scored 26 with 11 focus genes. Three additional networks identified by 


\section{Chapter 4}

IPA were of no importance since the score that they acquired was 1 and they are here not discussed further.

Table 2. Networks identified by IPA software of differentially expressed gene profiles obtained after exposure of human omental adipocytes to resistin, including the score from IPA, number of focus genes (= genes obtained via IPA as important in our dataset), top diseases and functions of the networks and the genes involved printing the focus genes that were differentially expressed in bold as well as the direction of their change in expression. Red arrows show upregulation and green arrows show downregulation.

\begin{tabular}{|c|c|c|c|c|}
\hline Network & Score & Focus genes & Top diseases and functions & Genes \\
\hline \multirow{3}{*}{1} & \multirow{3}{*}{29} & \multirow{3}{*}{12} & Protein trafficking & \multirow{3}{*}{ 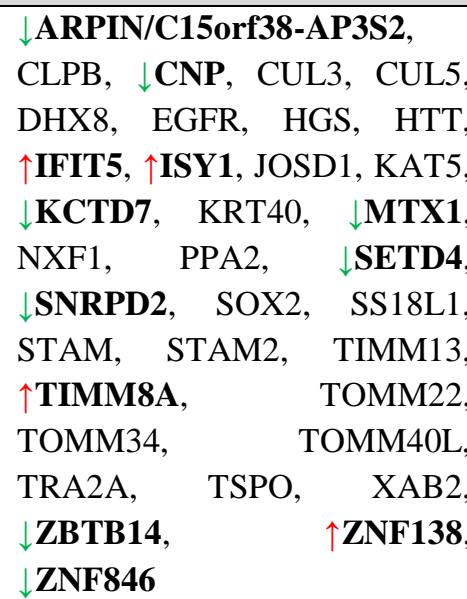 } \\
\hline & & & $\begin{array}{l}\text { Cellular Assembly and } \\
\text { organization }\end{array}$ & \\
\hline & & & $\begin{array}{l}\text { Cellular function and } \\
\text { maintenance }\end{array}$ & \\
\hline \multirow{3}{*}{2} & \multirow{3}{*}{26} & \multirow{3}{*}{11} & Cell Death and Survival & \multirow{3}{*}{ 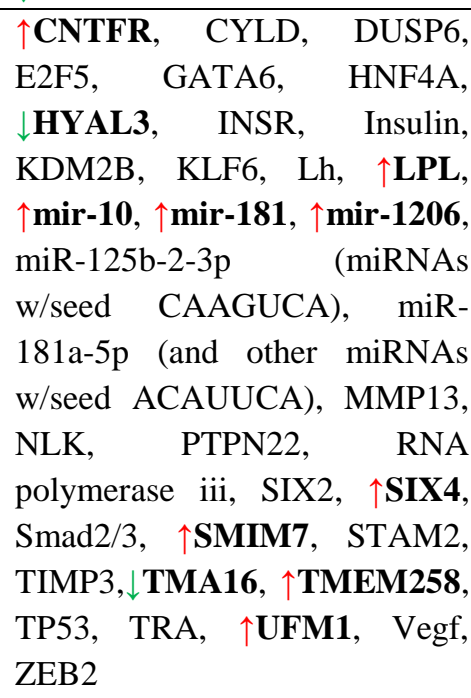 } \\
\hline & & & Cancer & \\
\hline & & & $\begin{array}{l}\text { Organismal Injury and } \\
\text { Abnormalities }\end{array}$ & \\
\hline
\end{tabular}

\section{Discussion}

In this study we analyzed the effect of exposure to resistin on gene expression in human omental adipocytes. Our study shows that several genes in the adipose cells were specifically and significantly affected in their expression by the presence of resistin. The proteins encoded by these genes are localized in different cell compartments, the nucleus, the mitochondrion as 
well as the cell membrane and cytosol. Most genes that were differently affected by resistin exposure encode proteins that are localized in the nucleus.

Analysis of the genes and proteins encoded shows that FLJ45340 is a protein with sequence similarity to tousled-like kinase 2, a nuclear serine/threonine kinase suggested to be involved in the regulation of chromatin assembly, the cell cycle and cell differentiation, SIX4 is a homeobox gene, involved in transcriptional regulation, ZNF138 is a zinc-finger protein involved in DNA binding and linked to RNA polymerase II, UFM1 is a ubiquitin-like protein, involved in protein regulation, CNTFR (Ciliary neurotrophic factor receptor) has a role in survival, differentiation and gene expression of neuronal cells. CNTFR is proposed to be involved in the IL-6 pathway (Pasquin et al., 2015; Schuster et al., 2003). IL-6 is one the adipocytokines with increased expression in obese adipose tissue (Cabia et al., 2016). ISY1 (ISY1 Splicing Factor Homolog) is involved in mRNA splicing. More specifically is involved in the first step of spliceosomal transesterification activity and in the biogenesis of primiRNA which in turn is a key factor in the developmental regulation of miRNA (Dix et al., 1999; Villa and Guthrie, 2005; Du et al., 2015).

The genes which are most significantly downregulated were SNRPD2, KCTD7, HYAL3 and SETD4. SNRPD2 is a small nuclear ribonucleoprotein, a protein required for pre-mRNA splicing and small nuclear ribonucleoprotein biogenesis, KCTD7 is a potassium channel protein which is involved in cell membrane hyperpolarization and HYAL3 is a hyaluronidaselike protein. Hyaluronidases are involved in degrading hyaluronan, a glycosaminoglycan thought to be involved in cell proliferation and differentiation. However, HYAL3 has not been shown to have hyaluronidase activity. The HYAL3 gene is located in a region on chromosome 3 with many genes associated with tumor suppression (Gerhard et al., 2004). SETD4 is a protein involved in ribosome biogenesis.

In addition to the genes most significantly up or down regulated discussed above, other genes of interest, are also significantly upregulated, albeit to lower extent. One of these genes encodes lipoprotein lipase (LPL). LPL functions as triglyceride hydrolase and as ligand/bridging factor for lipoprotein uptake (Klop et al., 2013). LPL plays also an important role in the processing of triglyceride-rich lipoproteins. More specifically it hydrolyzes circulating chylomicrons and very low density lipoproteins (VLDL) (Jung and Choi, 2014). Therefore the upregulation of LPL by resistin can lead to the increased number of free fatty acids in the blood, which might impair the proper storage of the fat. 


\section{Chapter 4}

The effects on a wide range of microRNAs shows the effect that resistin may have at the posttrancriptional regulation of gene expression. MicroRNAs are non-coding RNAs that can alter the post-transcriptional regulation of gene expression by affecting the stability and translation of mRNAs (NCBI).

Among the genes that are upregulated are also Piwi-interracting RNAs (piRNAs) (DQ598070 - piRNA-36136, DQ595173 - piRNA-61285, DQ596932 - piRNA-34998, DQ598479 piRNA-36545, DQ574699 - piRNA-35810) for which the function is still unclear. PiRNAs form complexes with piwi proteins that are related with both epigenetic and posttranscriptional gene silencing (Seto et al., 2007).

Also the function of C11orf10, C19orf42, MIR4731, SNORD113-1, MIR4800, SNORD1149, MIR611 and SNORD114-3 is still unknown and has to be further investigated. MIRs are microRNA's and SNORD are small nucleoar RNA's. The presence of these small RNA molecules suggests a possible regulation on mRNA stability or translation of mRNA into protein.

Besides the highly significantly downregulated genes discussed above other genes that are downregulated are interesting to discuss. JA429802 is a gene with high similarity to glutamine synthetase. Glutamine synthetase is important for nitrogen metabolism in cells and crucial for many fundamental processes as nucleotide synthesis. CHURC1-FNTB is proposed to be involved in the regulation of cell proliferation. SNORD25 is also downregulated in our study. This gene has been referred to as a guide RNA because it functions in the biogenesis of other small nucleolar RNAs. ZFP161 is long non-coding RNA (lncRNA) and is a transcriptional activator of dopamine (DAT) transporter and transcriptional repressor of Myc and thymidine kinase. Decrease of DAT has been related with aging (Bannon et al.,1992). Myc has been identified as proto-oncogene and is involved in cell proliferation, cell growth, apoptosis, stem cell self-renewal and cell competition (Denis et. al., 1991; Claveria et. al. 2013). C4orf43 is translation machinery-associated protein 16 involved in protein translation.

For other genes downregulated, the function is still unclear. These genes are LOC100133161, DQ596074, DQ592203, JA429802, C4orf43, DQ595216, U42379, DQ588370, JA535805, FLJ27352 and AK023614.

Overall the up- and down regulation of genes under the influence of resistin suggest modifications in transcriptional regulation processes with a suggested change, which is very 
likely a decrease, in cellular differentiation and maintenance processes in the presence of resistin.

Additionally, according to IPA software based pathway analysis performed for the 55 identified differentially expressed genes, the networks identified lead to the conclusion that resistin is also affecting lipid storage and usage. Altogether the results of the present study indicate that in the presence of resistin adipocytes downscale cellular processes with a concomitant decrease in energy expenditure. These changes in energy expenditure may be a reflection of the survival mode in which an organism is entering to cope with bad times in food supply, controlling lipid metabolism and assuring cellular maintenance. In modern societies where the access to food is easy the need of such an ancient surviving mechanism is redundant and may even turn into a harmful mode of action.

\section{References}

Bannon, M. J., et al. (1992). "Dopamine Transporter Messenger-Rna Content in Human Substantia-Nigra Decreases Precipitously with Age." Proc Natl Acad Sci U S A 89(15): 7095-7099.

Benomar, Y., et al. (2013). "Central Resistin Overexposure Induces Insulin Resistance Through Toll-Like Receptor 4." Diabetes 62(1): 102-114.

Caballero, A. E. (2003). "Endothelial dysfunction in obesity and insulin resistance: A road to diabetes and heart disease." Obesity Research 11(11): 1278-1289.

Cabia, B., et al. (2016). "A role for novel adipose tissue-secreted factors in obesity-related carcinogenesis." Obesity Reviews 17(4): 361-376.

Calle, E. E., et al. (2003). "Overweight, obesity, and mortality from cancer in a prospectively studied cohort of US adults." New England Journal of Medicine 348(17): 1625-1638.

Campo, G. M., et al. (2012). "The inhibition of hyaluronan degradation reduced proInflammatory cytokines in mouse synovial fibroblasts subjected to collagen-induced arthritis." Journal of Cellular Biochemistry 113(6): 1852-1867.

Claveria, C., et al. (2013). "Myc-driven endogenous cell competition in the early mammalian embryo." Nature 500(7460): 39-U53.

De Mars, G., et al. (2007). "Polymorphisms in the CNTF and CNTF receptor genes are associated with muscle strength in men and women." J Appl Physiol (1985) 102(5): 18241831.

Denis, N., et al. (1991). "Stimulation of Methotrexate Resistance and DihydrofolateReductase Gene Amplification by C-Myc." Oncogene 6(8): 1453-1457.

Dix, I., et al. (1999). "The identification and characterization of a novel splicing protein, Isy1p, of Saccharomyces cerevisiae." Rna-a Publication of the Rna Society 5(3): 360-368. 


\section{Chapter 4}

Du, P., et al. (2015). "A Biogenesis Step Upstream of Microprocessor Controls miR-17 similar to 92 Expression." Cell 162(4): 885-899.

Faria, J. A., et al. (2013). "SET domain-containing Protein 4 (SETD4) is a Newly Identified Cytosolic and Nuclear Lysine Methyltransferase involved in Breast Cancer Cell Proliferation." J Cancer Sci Ther 5(2): 58-65.

Freitas Lima, L. C., et al. (2015). "Adipokines, diabetes and atherosclerosis: an inflammatory association." Front Physiol 6: 304.

Galic, S., et al. (2010). "Adipose tissue as an endocrine organ." Mol Cell Endocrinol 316(2): 129-139.

Gerhard, D. S., et al. (2004). "The status, quality, and expansion of the NIH full-length cDNA project: the Mammalian Gene Collection (MGC)." Genome Res 14(10B): 2121-2127.

Hinman, J. D., et al. (2008). "Age-dependent accumulation of ubiquitinated 2',3'-cyclic nucleotide 3'-phosphodiesterase in myelin lipid rafts." Glia 56(1): 118-133.

Ingenuity Pathway Analysis. Available online: http://www.ingenuity.com

Jung, U. J. and M. S. Choi (2014). "Obesity and its metabolic complications: the role of adipokines and the relationship between obesity, inflammation, insulin resistance, dyslipidemia and nonalcoholic fatty liver disease." Int J Mol Sci 15(4): 6184-6223.

Kahn, B. B. and J. S. Flier (2000). "Obesity and insulin resistance." J Clin Invest 106(4): 473481.

Kawakami, K., et al. (1996). "Identification and expression of six family genes in mouse retina." FEBS Lett 393(2-3): 259-263.

Klop, B., et al. (2013). "Dyslipidemia in obesity: mechanisms and potential targets." Nutrients 5(4): 1218-1240.

Komatsu, M., et al. (2004). "A novel protein-conjugating system for Ufm1, a ubiquitin-fold modifier." Embo Journal 23(9): 1977-1986.

Lumeng, C. N. and A. R. Saltiel (2011). "Inflammatory links between obesity and metabolic disease." Journal of Clinical Investigation 121(6): 2111-2117.

McTernan, P. G., et al. (2002). "Increased resistin gene and protein expression in human abdominal adipose tissue." J Clin Endocrinol Metab 87(5): 2407.

Olefsky, J. M. and C. K. Glass (2010). "Macrophages, Inflammation, and Insulin Resistance." Annu Rev Physiol 72: 219-246.

Pasquin, S., et al. (2015). "Ciliary neurotrophic factor (CNTF): New facets of an old molecule for treating neurodegenerative and metabolic syndrome pathologies." Cytokine \& Growth Factor Reviews 26(5): 507-515.

Patel, L., et al. (2003). "Resistin is expressed in human macrophages and directly regulated by PPAR gamma activators." Biochem Biophys Res Commun 300(2): 472-476. 
Patel, S. D., et al. (2004). "Disulfide-dependent multimeric assembly of resistin family hormones." Science 304(5674): 1154-1158.

Reilly, M. P., et al. (2005). "Resistin is an inflammatory marker of atherosclerosis in humans." Circulation 111(7): 932-939.

Santos, S. H., et al. (2013). "Oral Angiotensin-(1-7) prevented obesity and hepatic inflammation by inhibition of resistin/TLR4/MAPK/NF-kappaB in rats fed with high-fat diet." Peptides 46: 47-52.

Schuster, B., et al. (2003). "Signaling of human ciliary neurotrophic factor (CNTF) revisited. The interleukin-6 receptor can serve as an alpha-receptor for CTNF." J Biol Chem 278(11): 9528-9535.

Schwartz, D. R. and M. A. Lazar (2011). "Human resistin: found in translation from mouse to man." Trends in Endocrinology and Metabolism 22(7): 259-265.

Seto, A. G., et al. (2007). "The coming of age for Piwi proteins." Molecular Cell 26(5): 603609.

Sheng, G. J., et al. (2003). "Churchill, a zinc finger transcriptional activator, regulates the transition between gastrulation and neurulation." Cell 115(5): 603-613.

Silha, J. V., et al. (2003). "Plasma resistin, adiponectin and leptin levels in lean and obese subjects: correlations with insulin resistance." European Journal of Endocrinology 149(4): 331-335.

Steppan, C. M., et al. (2001). "The hormone resistin links obesity to diabetes." Nature 409(6818): 307-312.

Tommerup, N. and H. Vissing (1995). "Isolation and Fine Mapping of 16 Novel Human Zinc Finger-Encoding Cdnas Identify Putative Candidate Genes for Developmental and Malignant Disorders." Genomics 27(2): 259-264.

Tycowski, K. T., et al. (1996). "A mammalian gene with introns instead of exons generating stable RNA products." Nature 379(6564): 464-466.

Vendrell, J., et al. (2004). "Resistin, adiponectin, ghrelin, leptin, and proinflammatory cytokines: Relationships in obesity." Obesity Research 12(6): 962-971.

Villa, T. and C. Guthrie (2005). "The Isy1p component of the NineTeen Complex interacts with the ATPase Prp16p to regulate the fidelity of pre-mRNA splicing." Genes \& Development 19(16): 1894-1904.

Wang, P., et al. (2004). "Profiling of the secreted proteins during 3T3-L1 adipocyte differentiation leads to the identification of novel adipokines." Cellular and Molecular Life Sciences 61(18): 2405-2417.

Wellen, K. E. and G. S. Hotamisligil (2003). "Obesity-induced inflammatory changes in adipose tissue." Journal of Clinical Investigation 112(12): 1785-1788.

WHO, Obesity and overweight. Fact sheet No311. 2015 



\section{Chapter 5}

\section{Identification of Type 2 Diabetes-related}

Biomarkers Derived from an in vitro Model of Inflamed Fat Tissue

J. P. ten Klooster
A. Sotiriou
S. Boeren
S. Vaessen
J. Vervoort
R.Pieters

Submitted for publication 


\title{
Chapter 5
}

\begin{abstract}
Currently, there is a worldwide increase of patients with Type 2 diabetes (T2DM). T2DM is highly correlated with elevated serum levels of IL6, TNF $\alpha$ and Hp and low levels of AdipoQ (Badawi et al., 2010). During the progression of healthy obese to T2DM status, there is an influx of immune cells, in particular macrophages, into adipose tissue, accompanied by an increase of inflammatory cytokines. To get a better insight in the underlying mechanisms, we performed a quantitative mass spectrometry analysis on a modified in vitro assay, combining 3T3L1 adipocytes and LPS activated RAW264.7 macrophages, thus mimicking inflamed adipose tissue. Clinically known markers, e.g. IL6, TNFa, AdipoQ, complement factor C3, B and $\mathrm{D}$ were identified, thus confirming the assay. In addition, we found 54 new proteins that can potentially be used for research into the mechanism of T2DM. Comparison of our results to a study on human visceral fat of obese non-diabetic and obese diabetic subjects, indicated that AUH, NAGK, pCYT2, NNMT, STK39 and CSNK2A2 have the potential of being prognostic biomarkers. Moreover, the expression of some of these genes was also changed in human blood samples at early stages of insulin desensitization. Overall, we conclude that the direct contact co-culture of 3T3L1 adipocytes with activated RAW264.7 macrophages is a mechanistically relevant and partially translational representative of inflamed visceral adipose tissue and can be used in identifying new "players" or biomarkers of T2DM regulation.
\end{abstract}




\section{Introduction}

In many Westernized cultures there is an increase in obesity, which may develop into metabolic syndrome and type 2 diabetes (T2DM) (Badawi et al., 2010; Arora et al., 2011). In obese subjects, an increase of lipid storage by adipocytes is observed (Badawi et al., 2010). In addition to lipid storage, adipose tissue can also have important endocrine and immunoregulatory functions (Badawi et al., 2010). Notably, by producing adipokines, hormones and inflammatory factors, adipose tissue can influence many processes in the human body (Hotamisligil et al., 1995; Spiegelman et al., 1993).

In the progression of healthy obese to a T2DM status the increase of the influx of immune cells, (especially macrophages) into adipose tissue is accompanied by release of inflammatory cytokines (TNF $\alpha$ and IL6 (Weisberg et al., 2003; Zhao et al., 2012)).

Although the role of IL6, CRP and Hp in insulin resistance is not clear, there are clear indications on the mechanism of insulin resistance caused by the macrophage-derived cytokine TNF $\alpha$ (Hotamisligil et al., 1996). Upon activation of the TNF $\alpha$ receptor, the adaptor molecule IRS1 will become phosphorylated on a serine residue, thus inhibiting tyrosine phosphorylation of this adaptor molecule. Tyrosine phosphorylation of IRS1 is required for relaying the signal coming from the insulin receptor towards the glucose transporter GLUT4, which will, subsequently, translocate to the plasma membrane and allow uptake of glucose (Hotamisligil et al. 1996). Indeed, studies in human patients suggest that fasting serum levels of glucose can be lowered by using an inhibitory anti-TNF $\alpha$ antibody (Gupta-Ganguli et al. 2011).

In order to treat $\mathrm{T} 2 \mathrm{DM}$ it is important to detect and prevent the onset of the disease as early as possible. However, the expression of most biomarkers which are currently used (AdipoQ, Hp, CRP, IL6, TNF $\alpha$ ) only change after serum glucose levels increase above normal levels, suggesting that they cannot be used as early and thus truly predictive biomarkers for onset of disease (Arora et al., 2011). Nevertheless, these biomarkers did help to clarify that T2DM is a metabolism-induced inflammatory disease (Arora et al., 2011). Many studies on biomarkeridentification have been performed, both in in vitro as well as in vivo models, with varying results (Badawi et al., 2010; Lopez-Villar et al., 2015).

Here, we used the co-culture system of 3T3L1 adipocytes and RAW264.7 macrophages, representing two cell types relevant in the onset of T2DM-related inflammation (Hotamisligil 


\section{Chapter 5}

et al., 1995; Spiegelman et al., 1993). We exposed the cultures to LPS, and subsequently analyzed protein expression by quantitative mass spectrometry analysis. We then verified the identified proteins in existing, published human patient data sets. This approach allowed us to identify new proteins/genes which may be used as early biomarkers for the development of T2DM in humans and help us to better understand the pathways that can lead to T2DM.

\section{Materials and Methods}

\section{Cell culture and 3T3L1 differentiation}

For all cultured cells, we used DMEM F12+ Glutamax medium (Gibco 31331-028) supplemented with $10 \%$ Fetal Calf Serum (Gibco 10082-147), 1\% Pen/Strep (Gibco 15070063), and 1mM sodium pyruvate (Gibco 11360-039). 3T3L1 (ATCC ${ }^{\circ}$ CL-173 ${ }^{\mathrm{TM}}$ ) and RAW264.7 (ATCC® TIB-71TM) cells were split 1:30 every 3 to 4 days. 3T3L1 preadipocytes were differentiated into adipocytes as described previously (Balvers et al., 2010). Briefly, cells were grown to confluency in a T75 bottle after which medium was replaced with fresh culture medium containing $1 \mu \mathrm{M}$ dexamethason (Sigma D4902), $10 \mu \mathrm{g}$ insulin $/ \mathrm{ml}$ (Sigma I0516) and $0.5 \mathrm{mM}$ IBMX (Sigma 1-5879). After 3 days, medium was replaced with culture medium supplemented with $10 \mu \mathrm{g}$ insulin $/ \mathrm{ml}$. Cells were allowed to differentiate for an additional 5 days after which cells were reseeded in collagen-coated (Advanced Biomatrix 5005-B) 6 well plates at a concentration of 500.000 cells per well. Cells were allowed to attach and grow for 2 additional days before exposure to RAW264.7 cells (50.000 cells per well) and E coli-derived LPS (Sigma L4391, $1 \mu \mathrm{g} / \mathrm{ml}$ for $18 \mathrm{~h}$ ) were started.

\section{RNA isolation and real-time PCR quantification}

Briefly, RNA from each sample was isolated (Zymo Research R1055) and 200 ng of RNA was used for cDNA synthesis by reverse transcription (Bio Rad 170-8891). Real-time PCR reactions included $5 \mu$ of diluted RT product (1:6 dilution), $10 \mu$ FAST SYBR Green buffer ( $\mathrm{AB} 4385614$ ) $4,8 \mu 1 \mathrm{H}_{2} \mathrm{O}$ and $0.5 \mu \mathrm{M}$ forward and $0.5 \mu \mathrm{M}$ reverse primer. Reactions were incubated in an Applied Biosystems 7500 Fast Real-Time PCR system in 96-well plates. The primers used are described in the Figure S1. Relative mRNA expression was determined by ddCT with HPRT mRNA expression as control.

\section{IL6 and TNFa ELISA}

Supernatants of exposed cells were collected and diluted 1:10 and 1:50 in ELISA dilution buffer according to manufacturer's protocol (R\&D cat nr DY206-05). Briefly, plates were 
coated with capture antibody for $18 \mathrm{~h}$, blocked for $1 \mathrm{~h}$, washed $3 \mathrm{x}$ and exposed for $2 \mathrm{~h}$ to 100 $\mu l$ diluted supernatant from exposed cells. Then plates were washed $3 \mathrm{x}$ and detection antibody was added for another $2 \mathrm{~h}$ after which the plates were washed again for $3 \mathrm{x}$ and streptavidinHRP was added for 30 minutes. Plates were washed again and HRP substrate (TMB, Sigma T4444) was added for 10-30 minutes after which the reaction was stopped by adding $50 \mu 1$ of $1 \mathrm{M} \mathrm{H} 2 \mathrm{SO} 4$. Colorimetric determination was performed in an ELISA plate reader at $450 \mathrm{~nm}$ wavelength.

\section{Mass spec analysis}

Four replicates of 3T3L1 adipocytes and RAW264.6 cells (untreated or LPS treated) were washed twice with PBS and then lysed in $250 \mu \mathrm{l}$ of $2 \mathrm{x}$ concentrated Laemli sample buffer. Samples were sonicated for $30 \mathrm{~min}$. Precast gels of $12 \%$ of Tris-glycine sodium dodecyl sulfate-polyacrylamide (SDS-PAGE) (Thermoscientific) were used for the separation of the proteins with gel electrophoresis. In each slot $35 \mu \mathrm{l}$ of sample-buffer (x5) diluted and boiled samples were added. The gel was stained with colloidal Coomasie blue (Colloidal blue staining kit, Thermoscientific). The gel was destained by washing twice with water. Each lane was cut into 5 equal pieces and placed in low protein-binding clean eppendorf tubes and were processed for in-gel digestion. Cysteine reduction was performed by adding $100 \mu \mathrm{l}$ of $50 \mathrm{mM}$ dithiotreitol (DTT) in $50 \mathrm{mM} \mathrm{NH} \mathrm{HCO}_{3}$ and incubated for $1 \mathrm{~h}$ at $60{ }^{\circ} \mathrm{C}$ by gently shaking. Alkylation followed by replacing DTT with $100 \mu \mathrm{l}$ of $100 \mathrm{mM}$ iodoacetamide (IAA) in 50 $\mathrm{mM} \mathrm{NH}_{4} \mathrm{HCO}_{3}$ and incubating for $1 \mathrm{~h}$ in room temperature by gently shaking. The enzymatic digestion was performed by adding $100 \mu \mathrm{l}$ of $10 \mathrm{ng} / \mu \mathrm{l}$ diluted in $50 \mathrm{mM} \mathrm{NH} \mathrm{NCO}_{3}$ and incubating overnight at room temperature. For the extraction of the proteins, samples were sonicated for $30 \mathrm{~s}$ and centrifuged shortly. Supernatants were transferred in clean low proteinbinding tubes. The $\mathrm{pH}$ was fixed between $2-4$ by adding $10 \%$ of TFA. The samples were analyzed by injecting $18 \mu \mathrm{l}$ sample over a $0.10 * 32 \mathrm{~mm}$ Prontosil 300-5-C18H (Bischoff, Germany) pre-concentration column (prepared in-house) at a constant pressure of 270 bar (normally resulting in a flow of ca. $7 \mu \mathrm{l} / \mathrm{min}$ ). Peptides were eluted from the pre-concentration column onto a $0.10 * 250 \mathrm{~mm}$ Prontosil 300-3-C18H analytical column (prepared in-house) with an acetonitrile gradient at a flow of $0.5 \mu \mathrm{l} / \mathrm{min}$ with a Proxeon EASY nanoLC. The gradient consisted of an increase from 9 to $34 \%$ acetonitrile in water with $1 \mathrm{ml} / \mathrm{l}$ formic acid in 50 minutes followed by a fast increase in the percentage acetonitrile to $80 \%$ (with $20 \%$ water and $1 \mathrm{ml} / \mathrm{l}$ formic acid in both the acetonitrile and the water) in 3 minutes as a column cleaning step. A P777 Upchurch microcross was positioned between the pre-concentration 


\section{Chapter 5}

and analytical column. An electrospray potential of $4.5 \mathrm{kV}$ was applied directly to the eluent via a stainless steel needle fitted into the waste line of the microcross. Full scan positive mode FTMS spectra were measured between $\mathrm{m} / \mathrm{z} 380$ and 1400 on a LTQ-Orbitrap (Thermo electron, San Jose, CA, USA) in the Orbitrap at high resolution (60000). CID MSMS scans of the four most abundant multiply charged peaks in the FTMS scan were recorded in data dependent mode in the linear trap (MS/MS threshold $=5.000)$.

\section{LC-MS analyses}

LC-MS runs with all MS/MS spectra obtained were analyzed with MaxQuant 1.3.0.5 (Cox et al., 2008) using default settings for the Andromeda search engine (Cox et al., 2008; Cox et al., 2011) except that extra variable modifications were set for de-amidation of $\mathrm{N}$ and $\mathrm{Q}$ as it was mentioned in a previous work (Arora et al., 2011). A mouse database downloaded from Uniprot (http://www.uniprot.org) was used together with a contaminants database that contains sequences of common contaminants as for instance: BSA (P02769, bovine serum albumin precursor), Trypsin (P00760, bovine), Trypsin (P00761, porcine), Keratin K22E (P35908, human), Keratin K1C9 (P35527, human), Keratin K2C1 (P04264, human) and Keratin K1CI (P35527, human). The "label-free quantification" as well as the "match between runs" (set to 2 minutes) options were enabled. De-amidated peptides were allowed to be used for protein quantification and all other quantification settings were kept default. Filtering and further bioinformatics analysis of the MaxQuant/Andromeda workflow output and the analysis of the abundances of the identified proteins were performed with the Perseus v.1.3.0.4 module (available at the MaxQuant suite). Accepted were peptides and proteins with a false discovery rate (FDR) of less than $1 \%$ and proteins with at least 2 identified peptides of which one should be unique and one should be unmodified. Reversed hits were deleted from the MaxQuant result table as well as all results showing a LFQ value of 0 for both LPS treated and untreated cells. Zero values for one of the two LFQ columns were replaced by a value of 4.5 to make sensible ratio calculations possible. Relative protein quantitation of sample to control was done with Perseus v1.3.0.4 by applying a two sample t-test using the normalized "LFQ intensity" columns obtained for LPS treated and untreated 3T3L1 adipocytes and RAW264.6 cells with threshold 0.05 and $\mathrm{S} 0=1$.

\section{Data analysis}

In vitro experiments were performed in triplicate and repeated at least three times in independent experiments. Data are presented as means with SD. Statistical analyses were performed on the data as depicted in the figures legends. 


\section{Results}

\section{Direct contact versus indirect contact between adipocytes and macrophages}

Currently, most publications on 3T3L1 adipocytes in co-culture with RAW264.7 macrophages describe indirect co-cultures by using transwell systems separating the adipocytes and the macrophages with a semi-permeable membrane that allows diffusion of cytokines and other cellular products, but prevents direct cellular contact (Sabater et al., 2013; Yamashita et al., 2007). In vivo, adipocytes and macrophages engage in direct cell-cell interactions and we postulate that this is important for the inflammatory response (Chatterjee et al. 2013). To test this, we differentiated 3T3-L1 pre-adipocytes into adipocytes for 1 week and subsequently reseeded the cells into collagen-coated wells and grew them for another week. Then, RAW 264.7 macrophages were added to the adipocytes directly (allowing cellcell contact) or indirectly by using a transwell or regular plates (no contact between macrophages and adipocytes). Cells were then co-cultured for $18 \mathrm{~h}$ in the absence or presence of LPS $(1 \mu \mathrm{g} / \mathrm{ml})$ to mimic an inflammatory response. We observed significantly higher levels of IL6 in the LPS-stimulated co-cultures with direct contact between adipocytes and macrophages when compared to LPS-stimulated co-cultures in which the cells were separated by a transwell membrane (Figure 1A). This was both observed by QPCR and ELISA, indicating that IL6 protein is newly synthesized. In addition, a similar effect was observed for IL1 $\alpha$ mRNA expression (Figure 1B). In contrast, the LPS-induced increase in TNF $\alpha$ mRNA expression appeared to be independent of cell-cell contact or even independent of adipocytes (Figure 1A), suggesting that the macrophages are the main source of TNF $\alpha$ in this co-culture. In addition, AdipoQ expression decreased after LPS in both direct and indirect co-cultures to similar levels (Figure 1C), suggesting that this decrease is independent of cell-cell contact.

\section{Identification of novel proteins involved in inflamed adipose tissue}

The established co-cultures of adipocytes and macrophages were then tested for changes in protein expression when stimulated with LPS for $18 \mathrm{~h}$. After exposure, cells were lysed and analyzed by LCMS (Figure 2A). From these analyses we observed 54 significantly changed proteins among which were $\mathrm{Hp}$ and complement factor B (CfB), which were both increased upon LPS exposure (Figure 2B). To confirm whether the increased Hp protein expression that we observed by mass spectrometry was due to increased gene activation, we performed microarray (Figure 2C) and QPCR (Figure 3A) analysis for the expression of Hp mRNA in 


\section{Chapter 5}
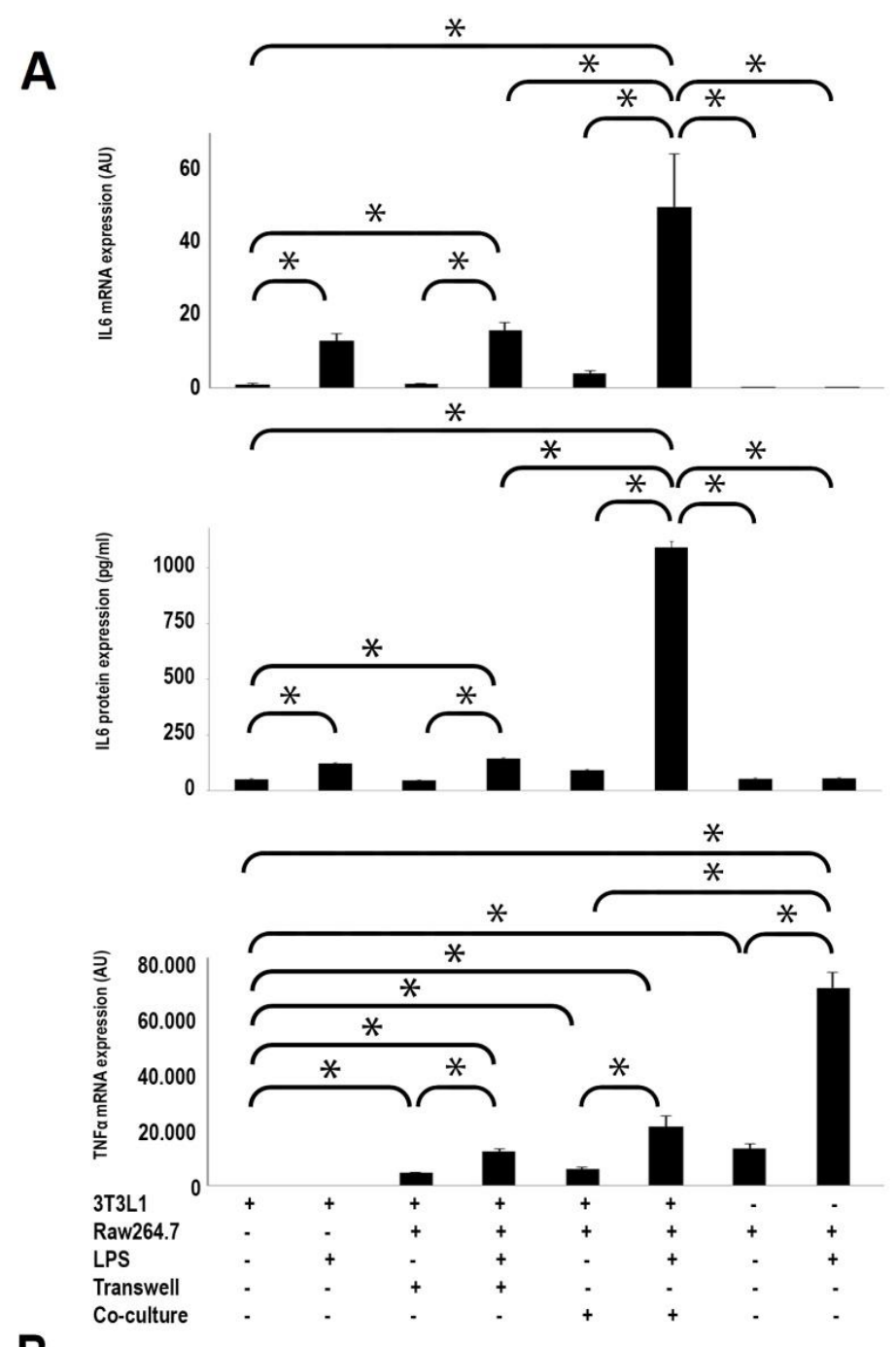

B

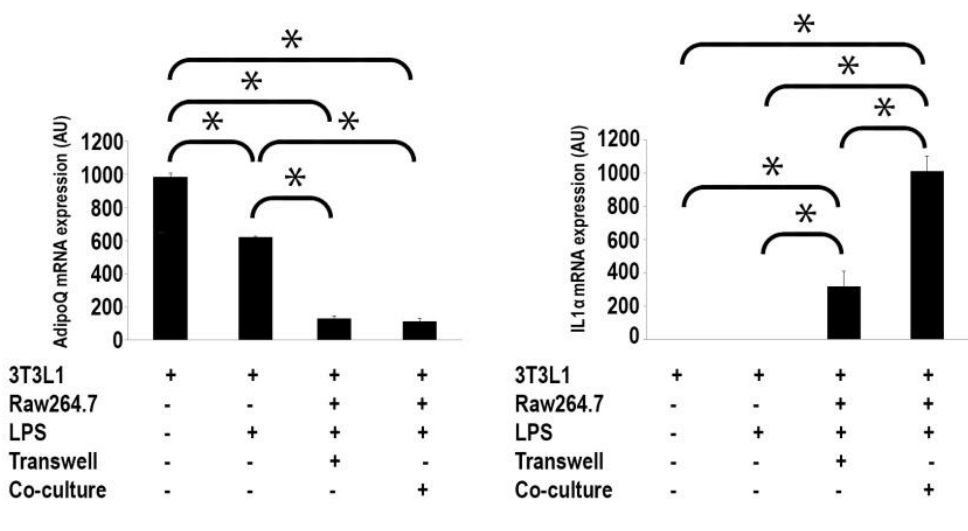

Figure 1. Direct contact between 3T3L1 adipocytes and RAW264.7 macrophages enhances inflammatory response. 3T3L1 adipocytes and RAW264.7 cells were either co-cultured with direct contact or separated by a transwell membrane. RAW264.7 cells were seeded in the upper compartment and the adipocytes in the lower compartment of the transwells. Cells were co-cultured and stimulated with LPS for $18 \mathrm{~h}$. (A) IL6 protein and mRNA and TNF $\alpha$ mRNA were normalized to untreated adipocytes. (B) IL1 $\alpha$ and AdipoQ mRNA were determined by QPCR and normalized to untreated 3T3L1 adipocytes. All experiments were performed 3 times (* indicates p-values which are lower than 0.01 with unpaired parametric Welch's t-test). 
adipocytes and macrophage co-cultures. Indeed, we observed an increase in $\mathrm{Hp}$ mRNA expression when adipocytes were stimulated with LPS, which appeared to be independent of macrophages (Figure 3A).

Also complement factor B was observed to be important (Figure 2B). In humans CfB and C3 expression levels were increased in patients with T2DM, whereas expression of adipsin was reported to be decreased in patients with T2DM (Wlazlo et al., 2014). The mRNA expression of $\mathrm{CfB}, \mathrm{C} 3$, and adipsin in our study reflects observations made in vivo in other studies. In addition, in agreement with literature(Wlazlo et al., 2014; Barbu et al., 2015; Vlaicu et al., 2016; van Greevenbroek et al., 2012; Moreno-Navarrete et al., 2010) and our ms data, CfB expression was increased when co-cultures of adipocytes and macrophages were stimulated with LPS for $18 \mathrm{~h}$ compared to non-stimulated co-cultures (Figure 3B). In contrast, C3 mRNA was increased in LPS-stimulated monocultures of adipocytes but not in monocultures of macrophages (Figure 3B), suggesting that the increase of C3 mRNA observed in the cocultures was, most likely, produced by the adipocytes.

When adipocytes were exposed to either macrophages or LPS, there was no significant decrease of adipsin observed on mRNA level. However, when adipocytes were stimulated with LPS in the presence of RAW264.7 macrophages, we observed a significant decrease of adipsin mRNA (Figure 3B). This decrease of adipsin is in line with lower levels of adipsin often found in human subjects suffering from T2DM (Derosa et al., 2013).

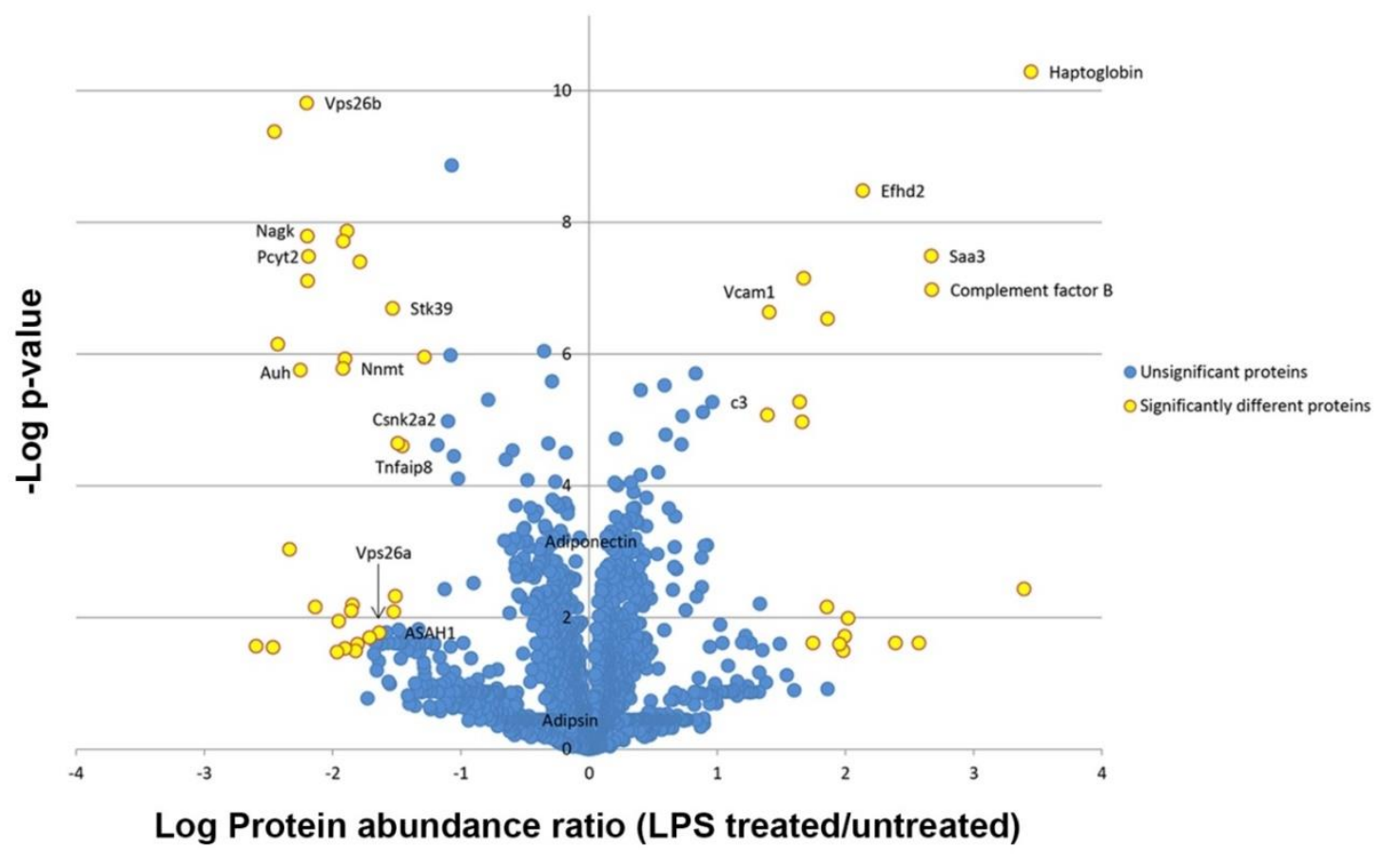


B

\begin{tabular}{|c|c|c|c|c|}
\hline Protein ID & Protein names & Gene names & ratio & $\begin{array}{l}\text {-Log t-test } \\
\text { p value }\end{array}$ \\
\hline Q3UBS3 & Haptoglobin & Нp & 3.45 & $\mathbf{1 0 . 2 9}$ \\
\hline E9Q5U3 & Glyceraldehyde-3-phosphate dehydrogenase & Gm10358 & 3.39 & 2.43 \\
\hline B8JJN0 & Complement factor $B$ & Gm20547 & 2.68 & 6.98 \\
\hline P04918 & Serum amyloid A-3 protein & Saa3 & 2.67 & 7.49 \\
\hline B7ZCQ3 & Non-histone chromosomal protein HMG-17 & Hmgn2 & 2.57 & 1.61 \\
\hline Q6ZQH8 & Nucleoporin NUP188 homolog & Nup188 & 2.39 & 1.61 \\
\hline Q8C845 & EF-hand domain-containing protein D2 & Efhd2 & 2.14 & 8.48 \\
\hline B2RTB0 & $28 \mathrm{kDa}$ heat- and acid-stable phosphoprotein & Pdap1 & 2.02 & 1.99 \\
\hline Q9Z1G4 & V-type proton ATPase $116 \mathrm{kDa}$ subunit a isoform 1 & Atp6v0a1 & 2.00 & 1.72 \\
\hline Q66JR8 & Ptms protein & Ptms & 1.98 & 1.50 \\
\hline A0JNY3 & Gephyrin & Gphn & 1.96 & 1.59 \\
\hline P15116 & Cadherin-2 & $\mathrm{Cdh} 2$ & 1.86 & 6.54 \\
\hline В1B079 & Zinc finger $\mathrm{CCCH}$ domain-containing protein 15 & Zc3h15 & 1.86 & 2.16 \\
\hline P17095 & High mobility group protein HMG-I/HMG-Y & Hmga1 & 1.75 & 1.61 \\
\hline Q3UGJ5 & Ras GTPase-activating protein 3 & Rasa3 & 1.67 & 7.15 \\
\hline P99027 & $60 \mathrm{~S}$ acidic ribosomal protein $\mathrm{P} 2$ & Rplp2 & 1.66 & 4.97 \\
\hline P54987 & Immune-responsive gene 1 protein & $\operatorname{Irg} 1$ & 1.64 & 5.27 \\
\hline P29533 & Vascular cell adhesion protein 1 & Vcam1 & 1.41 & 6.64 \\
\hline Q6IRU2 & Tropomyosin alpha-4 chain & Tpm4 & 1.39 & 5.08 \\
\hline Q541Z2 & Protein farnesyltransferase/geranylgeranyltransferase & Fnta & -1.18 & 4.62 \\
\hline A2AVJ6 & COMM domain-containing protein 9 & Commd9 & -1.28 & 5.96 \\
\hline D3Z312 & Tumor necrosis factor alpha-induced protein 8 & Tnfaip8 & -1.46 & 4.61 \\
\hline 054833 & Casein kinase II subunit alpha & Csnk2a2 & -1.49 & 4.64 \\
\hline Q9D967 & Magnesium-dependent phosphatase 1 & Mdp1 & -1.51 & 2.32 \\
\hline Q8R3Q6 & Coiled-coil domain-containing protein 58 & $\operatorname{Ccdc} 58$ & -1.52 & 2.09 \\
\hline A2AQL0 & STE20/SPS1-related proline-alanine-rich protein kinase & Stk39 & -1.53 & 6.70 \\
\hline Q3TWT5 & Acid ceramidase & Asah1 & -1.58 & 1.77 \\
\hline Q6P069 & Sorcin & Sri & -1.64 & 1.77 \\
\hline P67871 & Casein kinase II subunit beta & Csnk2b & -1.66 & 1.61 \\
\hline P40336 & Vacuolar protein sorting-associated protein $26 \mathrm{~A}$ & Vps26a & -1.67 & 1.62 \\
\hline Q3UBR8 & Mitochondrial import inner membrane translocase & Timm17b & -1.71 & 1.70 \\
\hline Q8VE96 & Transmembrane protein C2orf18 homolog & 4930471M23Rik & -1.79 & 7.40 \\
\hline Q9JKV1 & Proteasomal ubiquitin receptor ADRM1 & Adrm1 & -1.81 & 1.60 \\
\hline Q924D0 & Reticulon-4-interacting protein 1 , mitochondrial & Rtn4ip1 & -1.82 & 1.49 \\
\hline P50428 & Arylsulfatase A & Arsa & -1.84 & 2.19 \\
\hline A2AN96 & Putative hydrolase RBBP9 & Rbbp9 & -1.85 & 2.10 \\
\hline Q3TA37 & Elongation factor Ts & Tsfm & -1.89 & 7.87 \\
\hline Q01149 & Collagen alpha-2(I) chain & Col1a2 & -1.90 & 5.93 \\
\hline B1B0W8 & Hypoxanthine-guanine phosphoribosyltransferase & Hprt & -1.90 & 1.53 \\
\hline E9Q272 & Protein Hook homolog 3 & Hook3 & -1.92 & 7.71 \\
\hline 055239 & Nicotinamide N-methyltransferase & Nnmt & -1.92 & 5.78 \\
\hline Q7TNL5 & Serine/threonine-protein phosphatase $2 \mathrm{~A} 56 \mathrm{kDa}$ regulatory & Ppp2r5d & -1.95 & 1.94 \\
\hline Q542Y0 & $\mathrm{NAD}(\mathrm{P}) \mathrm{H}$ dehydrogenase [quinone] 1 & Nqo1 & -1.96 & 1.48 \\
\hline P20918 & Plasminogen & $\mathrm{Plg}$ & -2.13 & 2.16 \\
\hline Q3USD5 & Ethanolamine-phosphate cytidylyltransferase & Pcyt2 & -2.19 & 7.48 \\
\hline Q99LY9 & NADH dehydrogenase [ubiquinone] iron-sulfur protein 5 & Ndufs5 & -2.19 & 7.11 \\
\hline Q9D997 & $\mathrm{N}$-acetyl-D-glucosamine kinase & Nagk & -2.20 & 7.79 \\
\hline Q8C0E2 & Vacuolar protein sorting-associated protein 26B & Vps26b & -2.20 & 9.81 \\
\hline Q9JLZ3 & Methylglutaconyl-CoA hydratase, mitochondrial & Auh & -2.25 & 5.76 \\
\hline Q3UWG5 & CD81 antigen & $\mathrm{Cd} 81$ & -2.34 & 3.04 \\
\hline A2AMS1 & Sigma non-opioid intracellular receptor 1 & Sigmar1 & -2.43 & 6.15 \\
\hline P61082 & NEDD8-conjugating enzyme Ubc12 & Ube2m & -2.45 & 9.38 \\
\hline A3E4B1 & ATP synthase protein 8 & ATP8 & -2.46 & 1.55 \\
\hline O70456 & 14-3-3 protein sigma & Sfn & -2.60 & 1.57 \\
\hline
\end{tabular}



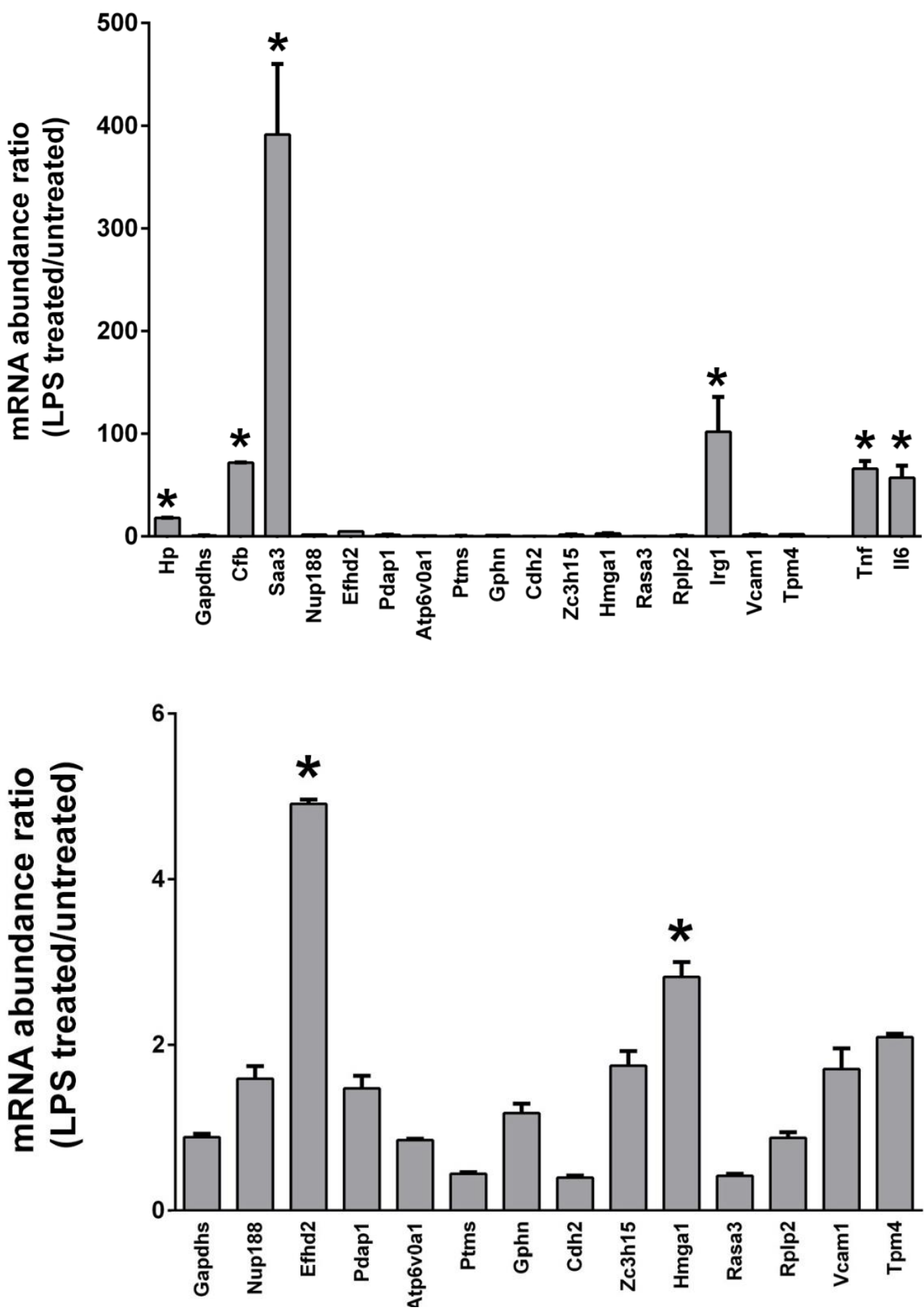


\section{Chapter 5}

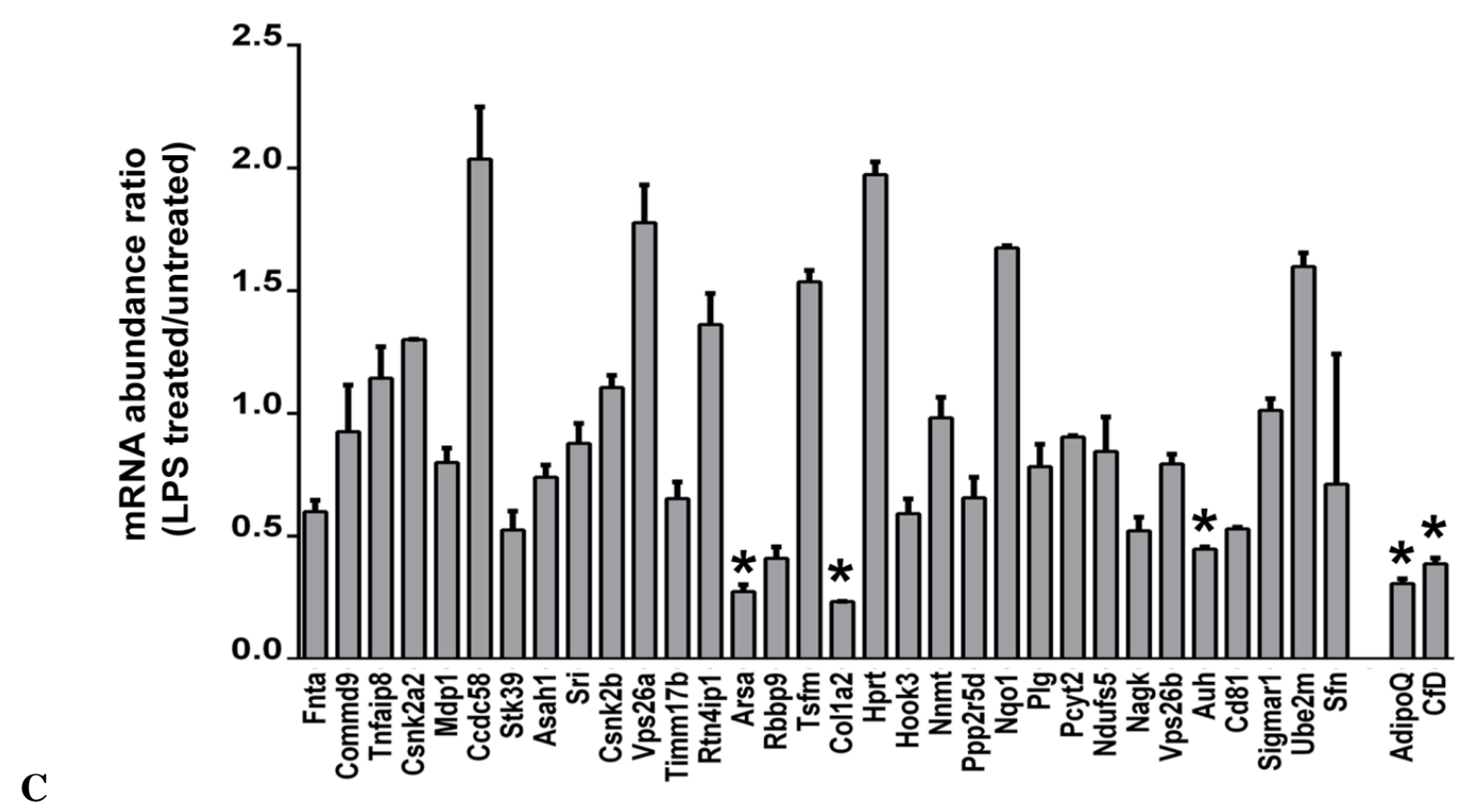

Figure 2. Quantitative mass spectrometry and mRNA analysis of LPS-stimulated co-cultures of 3T3L1 adipocytes and RAW264.7 macrophages. 3T3L1 adipocytes and RAW264.7 cells were co-cultured and stimulated with LPS $(1 \mu \mathrm{g} / \mathrm{ml})$ for $18 \mathrm{~h}$. (A) Vulcano plot of identified proteins in quantitative mass spectrometry, showing proteins that become upregulated upon LPS treatment on the positive right side and downregulated proteins on the negative left hand side in the figure. T-test p-values are shown on the y-axis as "Log p-value". (B) Table showing proteins (with their gene names) that have changed their expression after LPS stimulation as determined by quantitative mass spectrometry. Proteins that change their mRNA expression in humans are indicated in Bold. (C) mRNA expression, of proteins identified in quantitative mass spectrometry, in adipocytes versus co-cultures of adipocytes and RAW264.7 macrophages which were stimulated with LPS (1 $\mu \mathrm{g} / \mathrm{ml}$ ) for $18 \mathrm{~h}$. All experiments were performed 3 times (* indicates p-values which are lower than 0.01 with unpaired parametric Welch's t-test).

These observations suggest that a macrophage-derived factor might be responsible for the reduced expression of adipsin, possibly TNF $\alpha$ and/or IL6. Therefore, we tested whether IL6 or $\mathrm{TNF} \alpha$ themselves can alter the expression of $\mathrm{CfB}$, adipsin and $\mathrm{C} 3$ to the same level as observed with LPS stimulation in co-cultures of 3T3L1 and RAW264.7 cells. Indeed, when TNF $\alpha$ was added to 3T3L1 adipocytes, there was an increase of $\mathrm{CfB}$ and $\mathrm{C} 3$, and a decrease of adipsin to similar levels as observed for LPS-stimulated co-cultures, whereas IL6 did not affect the expression of these complement factors (Figure 3C). This suggests that TNF $\alpha$ is a key player in the regulation of complement expression by adipocytes. 
A

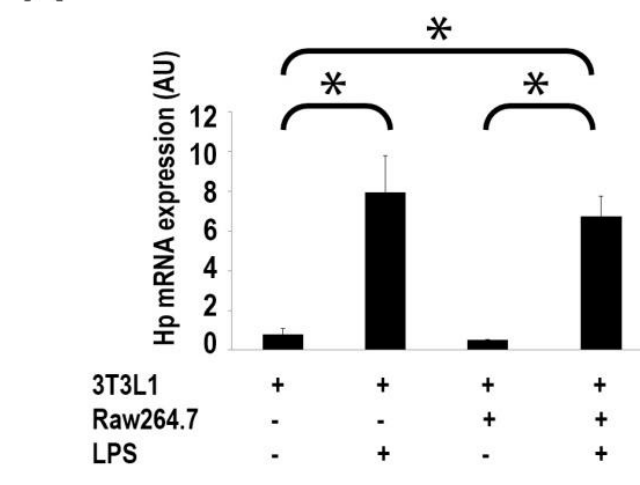

B
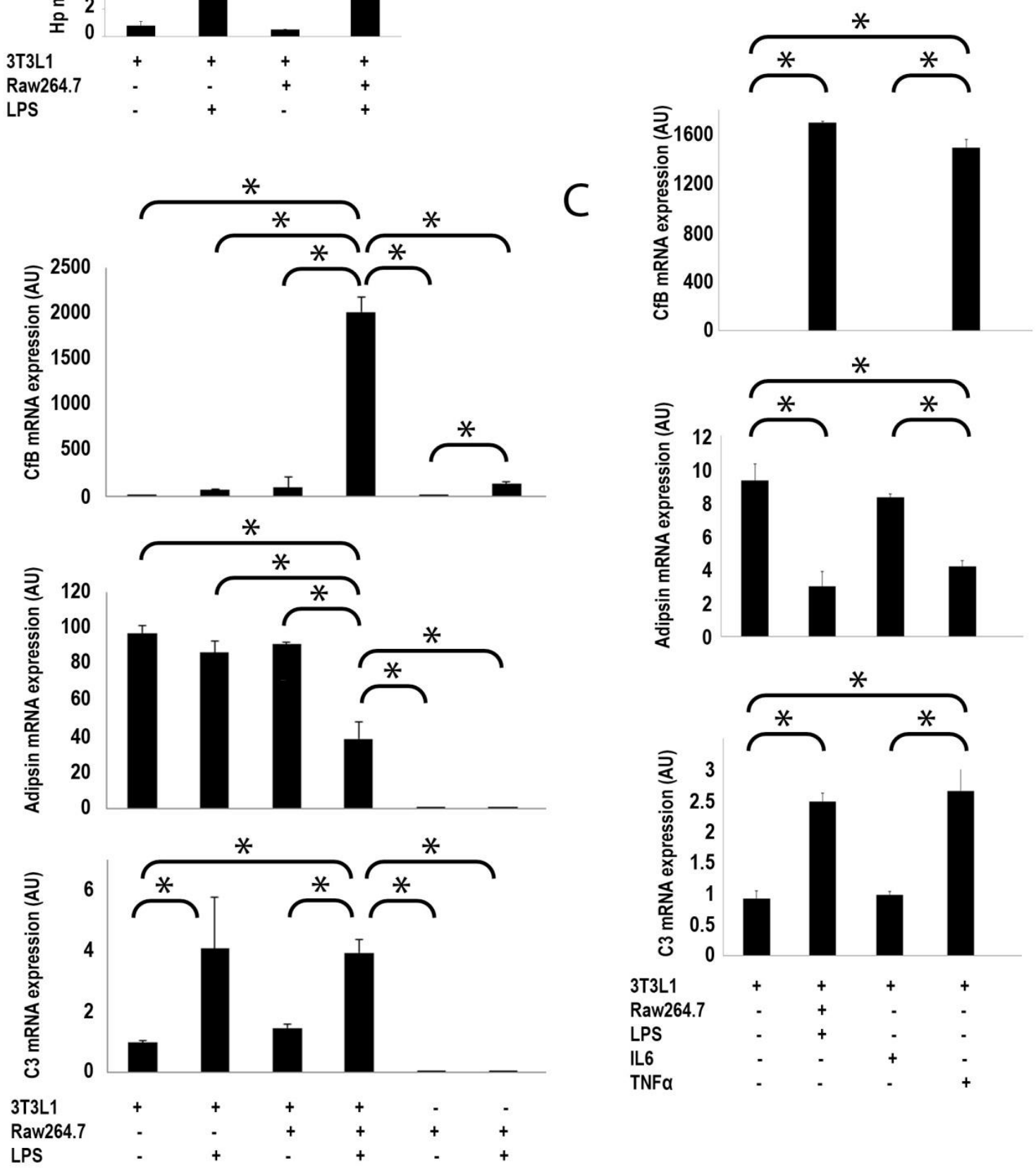

Fig $3 \mathrm{TNF} \alpha$ is a key-player in complement expression by 3T3L1 adipocytes and RAW264.7 macrophages. 3T3L1 adipocytes and RAW264.7 cells were co-cultured and stimulated with LPS $(1 \mu \mathrm{g} / \mathrm{ml})$ or TNF $\alpha(1 \mathrm{ng} / \mathrm{ml})$ for $18 \mathrm{~h}$ where indicated. (A) Hp mRNA was determined by QPCR and normalized to untreated adipocytes. (BC) $\mathrm{CfB}$, adipsin and $\mathrm{C} 3 \mathrm{mRNA}$ were determined by QPCR and normalized to untreated 3T3L1 adipocytes. All experiments were performed 3 times $(*$ indicates p-values which are lower than 0.01 with unpaired parametric Welch's t-test). 


\section{Chapter 5}

\section{Visceral adipose tissue in healthy obese versus T2DM obese women}

To further identify whether the protein hits from our mass spec analysis are relevant as biomarkers for human studies, we analyzed a publicly available human gene expression data set of visceral adipose tissue of 10 Indian obese women (Mathur et al. 2013). Five of these subjects were healthy obese and five were indicated as having T2DM. Both groups were matched for BMI. Expression of the 54 genes of the proteins we identified in our mass spec analysis as important with a notable difference between healthy obese and T2DM obese subjects are shown in Figure 4.

A decreased expression for VPS26A, VPS26B, pCYT2, AUH, STK39 and CSNK2A2 and an increase for VCAM1, EFHD2 and SAA1 (human orthologue for mouse SAA3), are in agreement with our mass spec data (Figure 4, Figure 2). However, NAGK and NNMT mRNA showed an increase in expression in vivo, whereas, we observed a decreased expression in vitro on protein level. Of note, not all of the 54 newly identified proteins, whose expression changed in our in vitro T2DM inflammatory model, showed an altered gene expression in the T2DM obese subjects compared to healthy obese subjects in the in vivo study on obese Indian women (Mathur et al. 2013). This could be due to differences between human versus mouse, in vivo versus in vitro or simply protein versus mRNA expression. To test the latter, we compared mRNA array data and proteomics data from 3T3L1 adipocytes and LPS-stimulated co-cultures of 3T3L1 adipocytes and RAW264.7 macrophages (Figure 2). For most of the genes that showed a difference in mRNA expression in diabetic versus non-diabetic obese women, we also observed a difference in mRNA expression in our in vitro model, suggesting that differences found between our in vitro proteomics data and the in vivo mRNA data from Mathur et al. (2013) is mainly because of differences between mRNA versus protein expression.

Currently, visceral fat is considered to have a larger impact on the development of T2DM than subcutaneous fat (Wajchenberg et al., 2002). To test whether the observed differences in gene expression were specific for visceral fat or can also be observed in subcutaneous fat from subjects suffering from T2DM, we analyzed the expression of the genes from figure $2 \mathrm{~A}$ as compared to a published study on human subcutaneous adipose tissue data sets from healthy and T2DM donors (Keller et al., 2011). Whereas we observed changes in gene expression levels upon T2DM development in visceral fat, no differences in gene expression were observed in subcutaneous fat from healthy obese versus T2DMobese patients (Figure 
S2). This accounted for all 54 newly identified genes (Figure S2) and AdipoQ (Figure S2), suggesting that the in vitro co-culture reflects visceral adipose tissue rather than subcutaneous adipose tissue (Figure S2).

\section{Some significant visceral adipose markers are reflected in blood}

When using biomarkers as indicators for disease progression, blood samples are more easily obtained than visceral adipose tissue samples. The proteins, observed in our study, can be either produced by adipocytes, macrophages or both. Although adipocytes, most likely, will not easily enter the blood or lymphatic system, the more motile macrophages can. So therefore, we analyzed in another publicly available dataset (Karolina et al., 2011) whether the expression of the genes from Figure 2 is significantly changed in blood samples from obese male adult human subjects with either a normal fasting glucose level, an impaired fasting glucose (IFG) level or diagnosed as T2DM patient. As a control, we first checked whether the expression of currently used biomarkers, such as AdipoQ and Hp (Badawi et al., 2010; Quaye et al., 2008; Chiellini et al., 2004; Lisi et al,. 2011; Maffei et al., 2016), were changed in the blood cell analyses of that study. Indeed, expression of AdipoQ and Hp decreased and increased, respectively, in subjects with decreased insulin sensitivity or T2DM (Figure 5). Although not all genes in blood changed their expression as compared to our analysis on visceral fat, we noted a downregulation of pCYT2 (expressed highly by RAW264.7), STK39, NAGK and SAA1 and a slight up-regulation of NNMT and AUH mRNA in blood of subjects with IFG and T2DM (Figure 5). This suggests that products of these genes can potentially be used as prognostic markers for insulin sensitivity. 

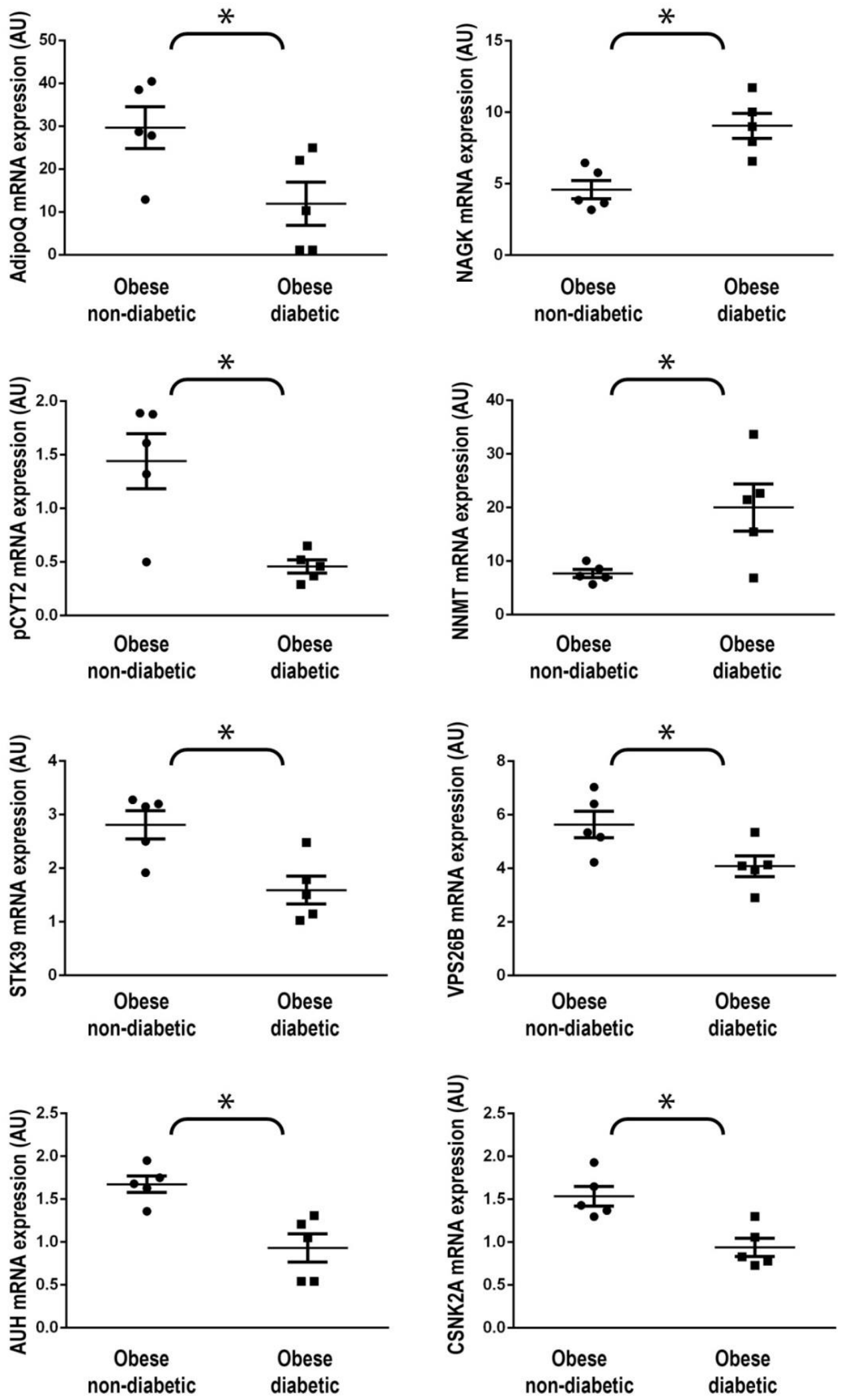

Figure 4. mRNA expression in obese non-diabetic and obese diabetic Indian women of genes identified in quantitative mass spectrometry. Through literature data mining we determined the mRNA expression of genes identified through proteomics in 5 obese non-diabetic and 5 obese diabetic women. Every data point represents 1 subject (* indicates p-values which are lower than 0.05 with non-parametric t-test according to Mann-Whitney). 

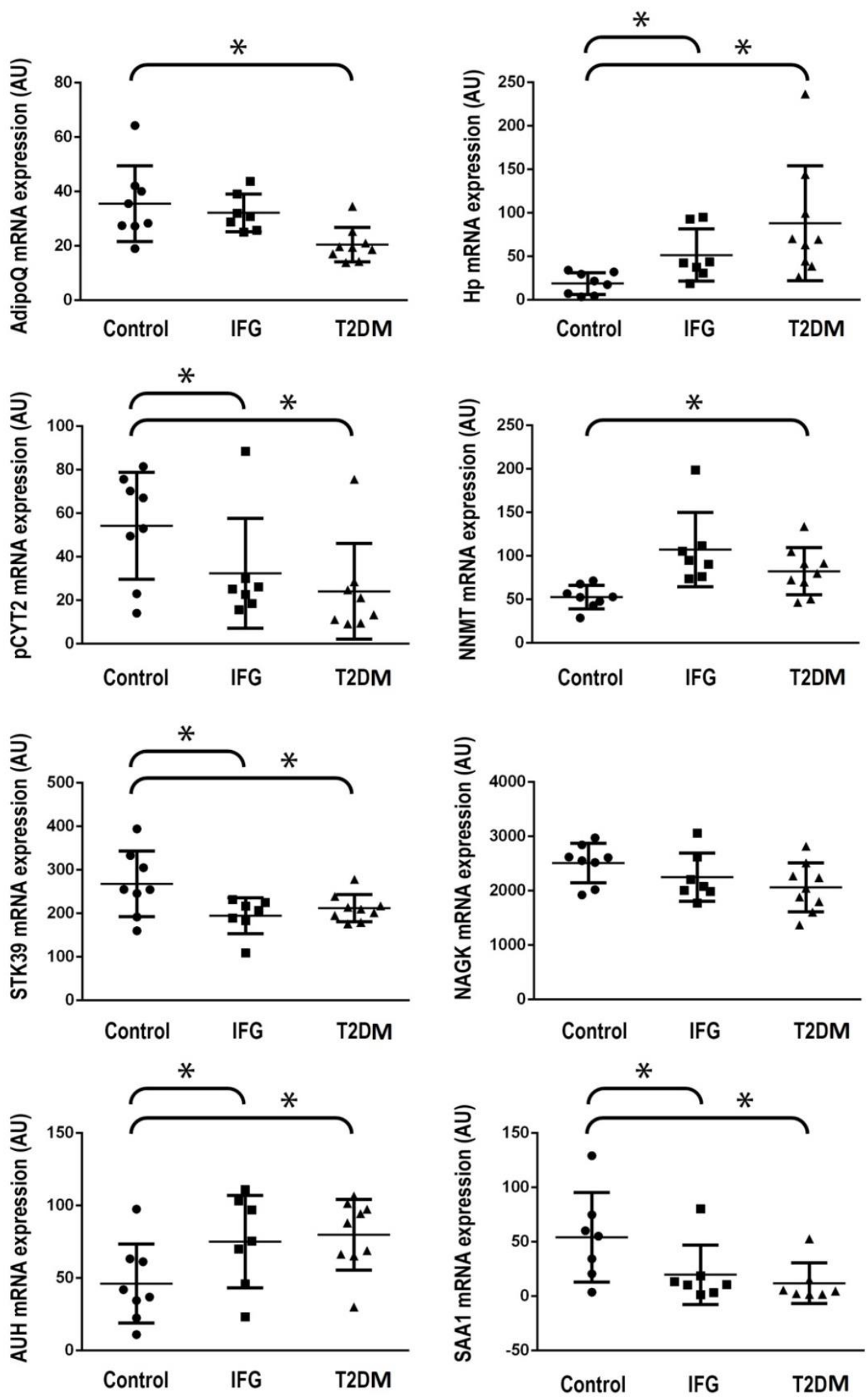

Figure 5. mRNA expression in blood samples of healthy obese, IFG and T2DM subjects of genes identified in quantitative mass spectrometry. Through literature data mining we determined the mRNA expression, of genes identified through proteomics, in blood samples which were obtained from obese male adult human subjects (21 to 70 years of age) with normal fasting glucose $(<6.1 \mathrm{mmol}$ glucose/L), impaired fasting glucose (IFG) (6.0 mmol glucose/1 - $7.0 \mathrm{mmol}$ glucose/L) and T2DM (>7.0 mmol glucose/L) (Karolina et al., 2011). Every data point represents 1 subject (* indicates p-values which are lower than 0.05 with non-parametric t-test according to Mann-Whitney). 


\section{Chapter 5}

\section{Druggable targets in co-culture of adipocytes and macrophages.}

To test whether our in vitro model can be used for drug screening we tested rosiglitazone. Rosiglitazone is known for its beneficial effect on insulin sensitivity in humans by activating PPAR $\gamma$ and increasing AdipoQ (Starner et al., 2008). To test whether rosiglitazone can affect the expression of IL6, TNF $\alpha, \mathrm{Hp}$, AdipoQ or adipsin in the in vitro co-cultures, we exposed 3T3L1 adipocytes, RAW264.7 and 3T3L1/RAW264.7 co-cultures to rosiglitazone during LPS stimulation analyzing the expression of IL6, TNF $\alpha, \mathrm{Hp}$, AdipoQ or adipsin. After an overnight exposure, ELISAs for IL6 and TNFa (Figure 6A) indicated that rosiglitazone inhibited LPS-induced IL6 and TNF $\alpha$ secretion, which points to a beneficial effect for insulin sensitivity.

The rosiglitazone-samples were tested for mRNA expression of Hp, AdipoQ and adipsin by QPCR (Figure 6B) and noticed that, similar to what is observed in humans, rosiglitazone can increase AdipoQ mRNA expression in adipocyte mono-cultures (Figure 6B). However, this beneficial effect of rosiglitazone on AdipoQ is completely abolished when adipocytes were co-cultured with macrophages in the presence of LPS. In addition, rosiglitazone caused a higher Hp mRNA expression but could not prevent the LPS-induced decrease of adipsin (Figure 6B). This suggests that rosiglitazone can affect T2DM through multiple pathways. 
A
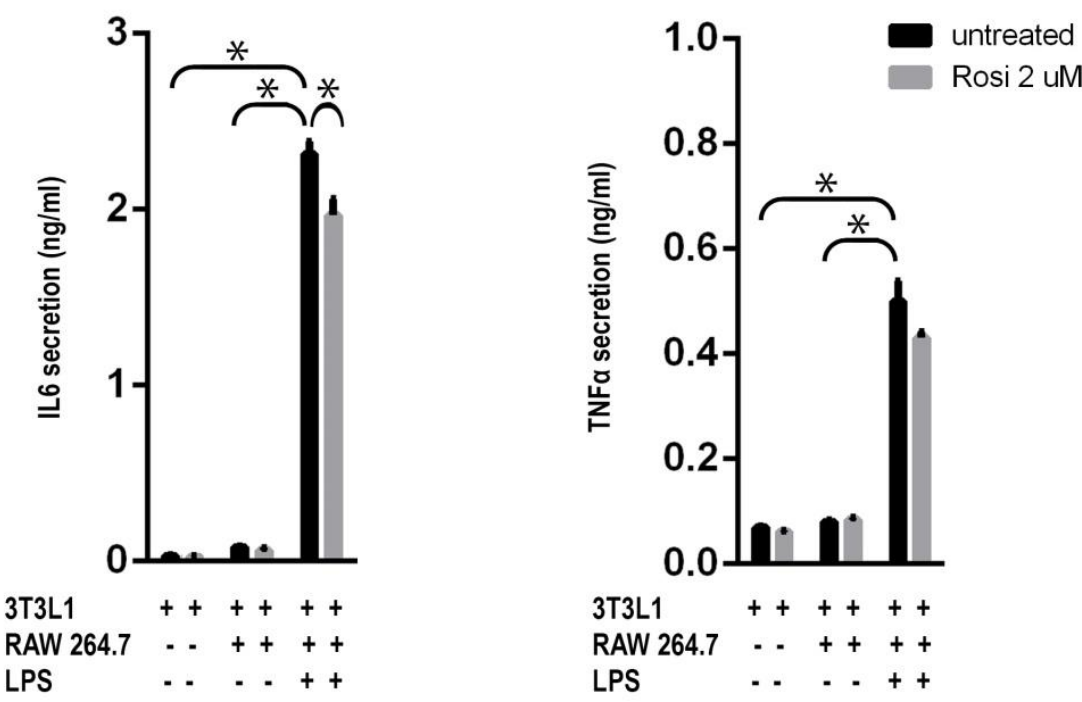

B
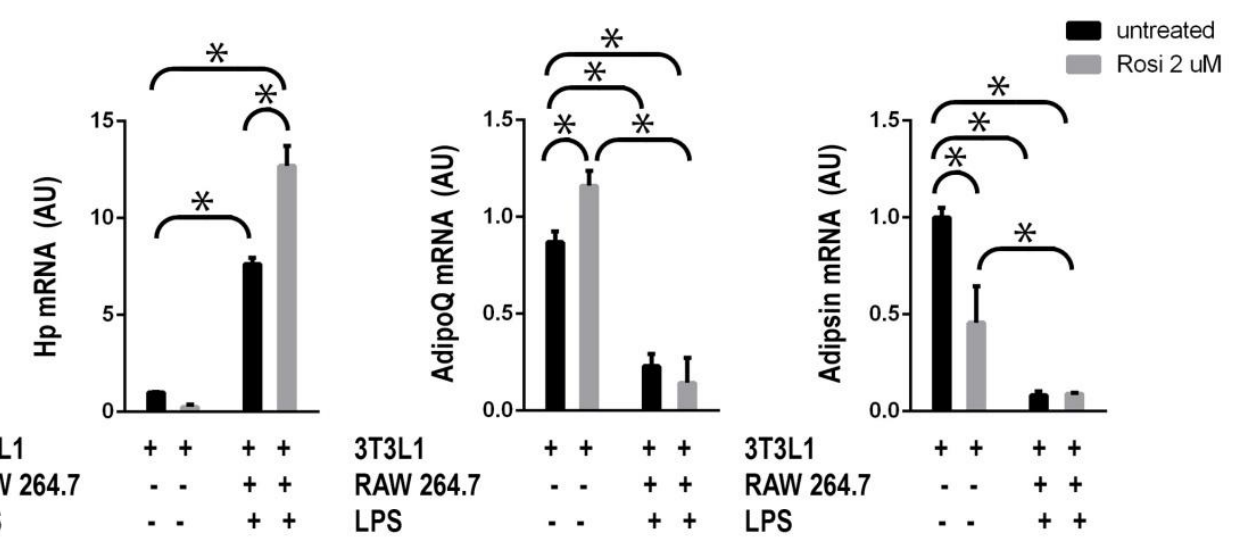

Fig 6 Involvement of PPAR $\gamma$ agonist in adipocyte inflammation. 3T3L1 adipocytes and RAW264.7 cells were co-cultured and stimulated with LPS $(1 \mu \mathrm{g} / \mathrm{ml})$ for $18 \mathrm{~h}$ where indicated. (A) IL6 and TNF $\alpha$ protein concentrations were determined by ELISA and normalized to untreated adipocytes. (B) Hp, AdipoQ and adipsin mRNA expression was determined by QPCR normalized to untreated adipocytes (* indicates p-values which are lower than 0.01 with unpaired parametric Welch's t-test).

\section{Discussion}

Through mass spec analysis on an in vitro co-culture of macrophages and adipocytes we have shown that this model might be helpful in researching many aspects of T2DM development as indicated by the altered expression of known biomarkers, such as AdipoQ, Hp, TNF $\alpha$ and IL6 (Badawi et al., 2010; Hotamisligil et al., 1996; Maffei et al., 2016; Xita and Tsatsoulis, 2012). Importantly, most of the known biomarkers for T2DM are produced by visceral adipose tissue rather than by subcutaneous adipose tissue (Badawi et al., 2010; Wajchenberg et al., 2002) 


\section{Chapter 5}

(Figure S2). Both protein and mRNA expression profiles of these known biomarkers, confirm that our in vitro co-culture system has more overlap with visceral adipose tissue then subcutaneous adipose tissue. Also newly identified proteins (a.o. pCYT2, NNMT, STK39, NAGK, SAA1, VPS26A\&B and AUH) in our study were confirmed by comparison to a published study on human mRNA expression. These proteins have the potency to be used as biomarkers for T2DM progression. The observed biomarkers can be linked to pathways for a better understanding of the development of $\mathrm{T} 2 \mathrm{DM}$ and to design specific therapeutic approaches (Figure 7). Here, we will discuss the potential roles of the proteins in T2DM we observed to change their expression in vitro and in vivo (Figure 7). First we will discuss key regulator TNF $\alpha$ followed by other biomarkers from this study that are promising but with a hitherto less well known role in T2DM development.

\section{TNFa}

A key molecule in insulin desensitization is $\mathrm{TNF} \alpha$, well known to inhibit insulin receptor signaling pathways by blocking IRS1 function, and subsequently GLUT4-mediated glucose transport across the plasma membrane (Hotamisligil et al., 1995). Although TNF $\alpha$ is an important factor in insulin resistance it is still not clear whether other pathways emanating from the TLR4 receptor might also play an important role in T2DM. Hence, in the co-cultures of adipocytes and macrophages we induced TNF $\alpha$ expression through activation of TLR4 by LPS. Besides its effect on IRS1 functioning, it is also possible that TNF $\alpha$ has other ways of affecting diabetes. Indeed, in our studies we show that $\mathrm{TNF} \alpha$ regulates the expression of $\mathrm{CfB}$, C3, and adipsin in 3T3L1 adipocytes (Figure 3). Adipsin from adipocytes, which is downregulated by $\mathrm{TNF} \alpha$, is involved in the production of $\mathrm{C} 3 \mathrm{a}$ from $\mathrm{C} 3$, the first being a recently newly identified secretagogue for insulin (Lo et al., 2014). Although we observed an increase of $\mathrm{C} 3 \mathrm{mRNA}$, we found a decrease in adipsin mRNA, suggesting that less C3a will be produced after TNF $\alpha$ exposure. This indicates that TNF $\alpha$ might not only be involved in desensitizing the insulin receptor but also in regulating insulin secretion by the pancreas.

Although $\mathrm{TNF} \alpha$ can induce the expression of most biomarkers, it is striking that the expression of TNF $\alpha$ is lower in the LPS-stimulated co-cultures of 3T3L1 and RAW246.7 then in RAW264.7 mono-cultures, whereas the induced expression and secretion of IL6 and IL1 $\alpha$ is much higher in the direct contact co-cultures. This suggests that TNF $\alpha$ is not the only protein involved in the inflammatory response of these co-cultures. 


\section{pCYT2}

One of the down-regulated proteins upon LPS stimulation of adipocyte/macrophage cocultures was pCYT2, an enzyme involved in de novo biosynthesis of $\mathrm{PE}$ (phosphatidylethanolamine) from ethanolamine and diacylglycerol. (Pavlovic et al., 2013). $\mathrm{pCYT2}^{+/-}$mice, containing less of this enzyme, show elevated lipogenesis and lipoprotein secretion, hypertriglyceridemia, obesity, and insulin resistance (Fullerton et al., 2009). This suggests that decreased expression of pCYT2 as a result of inflamed adipose tissue, could be one of the underlying mechanisms involved in T2DM development.

\section{NNMT}

Another protein whose expression decreased after LPS treatment in vitro was NNMT (Figure 2).The mRNA expression of NNMT, on the other hand, was increased in obese non-diabetic $v s$. obese diabetic Indian women (Figure 4). Recently, the importance of NNMT for T2DMrelated health effects was demonstrated by showing that NNMT expression was markedly increased in adipose tissue of GLUT4 $4^{-/-}$mice (Kraus et al., 2014). Moreover, NNMT knockout $\left(\mathrm{NNMT}^{--}\right)$mice were protected against diet-induced obesity and showed improved glucose tolerance while inhibition of NNMT increased adipose NAD+ levels which is related to energy metabolism (Kraus et al., 2014). The finding in the human study with Indian women is in agreement with the data observed in $\mathrm{NNMT}^{-/-}$mice. The discrepancy of these in vivo findings with the observed decreased NNMT expression in our in vitro model cannot be explained by a difference between mRNA and protein, because the NNMT mRNA expression in our LPS-stimulated in vitro co-cultures did not change compared to non-stimulated cocultures (Figure 2). Perhaps, the decreased expression found in vitro is a result of a short-term TLR4 stimulation, as cells may behave differently when cells are exposed to TLR4 agonists, such as LPS or Fetuin/Free Fatty Acids complex (Pal et al., 2012), for a longer period, e.g. weeks or months, which might be the case in vivo.

\section{STK39}

A third protein we found down-regulated after treating adipocyte/macrophage co-cultures with LPS was STK39/SPAK (Figure 2 and 4). The serine/threonine kinase STK39/SPAK is part of the WNK (With-No-Lysine)/SPAK/NCC pathway that regulates sodium and potassium transport across the plasma membrane (Moriguchi et al., 2005; Gagnon and Delpire, 2010). Besides changes in expression of STK39/SPAK, it has been observed that 


\section{Chapter 5}

STK39/SPAK and its substrate NCC (Na-Cl co-transporter), are highly phosphorylated in hyperinsulinemic $\mathrm{db} / \mathrm{db}$ mice compared to non-diabetic $\mathrm{db} / \mathrm{db}$ mice (Nishida et al., 2012), indicating a role for STK39 and NCC in the development of T2DM. In addition, clinical studies with the NCC specific inhibitor, thiazide, used to prevent high blood pressure, showed that patients with diabetes increase their fasting glucose and decrease their insulin sensitivity after administration of thiazide, indeed suggesting that the WNK/SPAK/NCC-pathway is involved in increasing insulin resistance (Eriksson et al., 2008; Hirst et al., 2015). Besides affecting NCC, STK39/SPAK can also inactivate the chloride-transporter CFTR, which is involved in Cystic Fibrosis (Yang et al., 2011). This raises the question whether this transporter might be involved in diabetes. Recently, a new CFTR-activating drug (Ivacaftor) was developed, which is currently used as a treatment for a sub-population of CF patients and preliminary data with Ivacaftor show that CF patients suffering from the first stage of diabetes revert their fasting glucose levels to normal when treated with this drug (Bellin et al. 2013). This indeed suggests that the WNK/STK39/NCC pathway might be involved in T2DM development and can be a potential druggable target (Bellin et al. 2013).

\section{NAGK}

NAGK was found to be upregulated after LPS treatment in vitro and in vivo (Figure 2 and 4) but has never been functionally linked directly to T2DM. NAGK is a kinase that phosphorylates N-acetyl-glucosamine (GlcNac) (Berger et al., 2002; Weihofen et al., 2006) and it has been described that increased O-linked beta-N-acetylglucosamine (O-GlcNAc) is associated with insulin resistance in muscle and adipocytes (Copeland et al., 2008; Myslicki et al., 2014). In addition, GlcNac might be involved in insulin receptor signaling by regulating the interaction between PI3K and the Insulin Receptor Substrates 1 and 2 (IRS1 and IRS2)(Klein et al., 2009; Whelan et al., 2010), suggesting that GlcNac and perhaps its kinase NAGK play an important role in insulin signaling.

\section{SAA}

The human acute-phase serum amyloid A protein 1 (hSAA1) is produced in adipose tissue (Sjoholm et al., 2005) and, in agreement with our data, the expression of this inflammatory adipokine correlates with metabolic complications, such as atherosclerosis and insulin resistance (Yang et al., 2006). In our study, we used LPS-mediated activation of TLR4 as a trigger for insulin resistance, however, in vivo the expression of mSAA3 (homologue of hSAA1) can also be triggered by a high fed-diet (HFD) and the expression of mSAA3 
correlates with the onset of insulin resistance (Scheja et al., 2008). Moreover, exposure of 3T3L1 adipocytes to SAA3, caused alterations in gene expression, which closely resembled those observed in HFD-mice (Scheja et al., 2008).

\section{VPS26B}

VPS26B is another protein that was down-regulated in our co-culture system after LPS exposure. VPS26A and B are part of the prototypical heteropentameric mammalian retromer (sorting nexin (SNX)-BAR retromer) complex that regulates the translocation of GLUT4 from endosomes to the Trans-Golgi-Network (TGN) (Ma et al., 2014). When expression of VPS26 is lost, GLUT4 is no longer transported to the TGN but rather redirected to lysosomes, where it will be degraded (Ma et al., 2014). This redirection is dependent on CSNK2-activity and inhibition of CSNK2 blocks the lysosomal targeting and degradation of GLUT4 (Ma et al., 2014). In our study, both VPS26 and CSNK2 are down regulated in protein and mRNA expression in vitro (Figure 2) and in vivo (Figure 4), suggesting that VPS26 and CSNK2 are important factors in reduction of glucose transport.

\section{AUH}

Finally, the protein expression of AUH, an RNA-binding protein with enoyl-CoA hydratase activity (Nakagawa et al., 1995), decreases upon LPS stimulation in co-cultures of adipocytes and macrophages. However, the mRNA expression of this gene is upregulated in blood samples of subjects that have increased glucose levels (Figure 5). Currently, there are no papers describing a role for this protein in diabetes, however, this crotonase can hydrate the double bond between the second and third carbons in acyl-CoA (Nakagawa et al., 1995), suggesting that it might be involved in lipogenesis and thus has the potency of linking AUspecific RNA elements to metabolism.

3T3L1 adipocytes have been used in many studies for unraveling the pathways of adipogenesis, insulin signaling and T2DM development. Most of these studies focus on known targets or try to identify new genes, based on mRNA expression profiles by microarray-based techniques (Vikman et al., 2014; Dehwah et al., 2012; Mori et al., 2012), whereas only a few papers have been published with respect to proteomics on adipose tissue (Lopez-Villar et al., 2015; Fang et al., 2015). In this paper, we show that many relevant genes change their protein expression without significantly changing their mRNA levels (Figure 2). 


\section{Chapter 5}

The co-cultures of adipocytes and macrophages have expression profiles that are more comparable to visceral then subcutaneous adipose tissues (Figure 4, S2) and, as such, are very interesting as an in vitro model for drug screening (Figure 6) and research on signaling pathways involved in insulin sensitivity (Figure 1, 2 and 7). In addition, we identified genes that are known factors in development of atherosclerosis indicating that this in vitro model might also help to clarify the role of inflamed adipose tissue in other aspects of the metabolic syndrome and might not be restricted to diabetes.

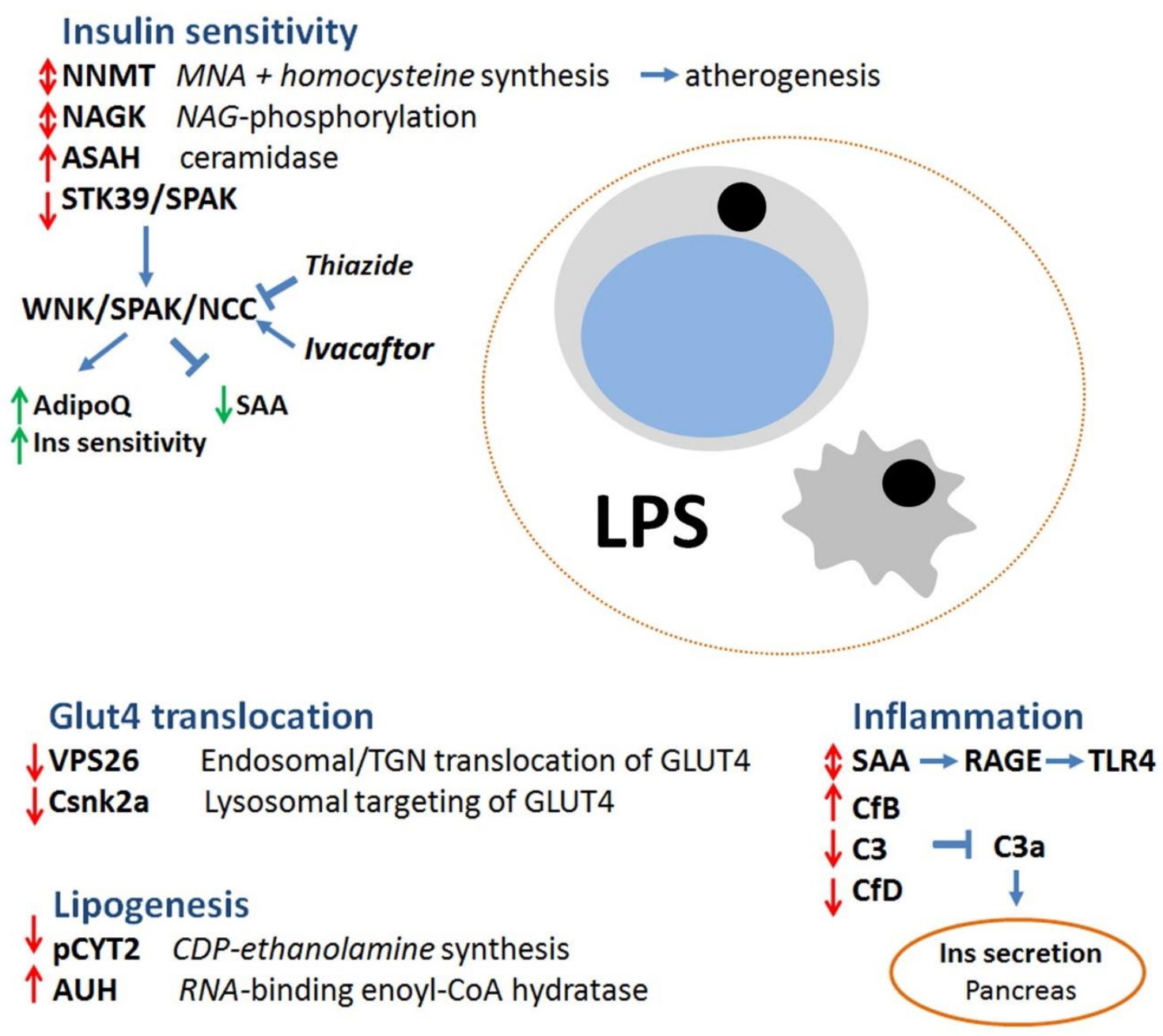

Figure 7. Schematic overview of identified proteins. Inflamed adipose tissue is in this model represented by coculture of adipocytes and macrophages, stimulated with LPS. Red arrows indicate the change in expression after LPS-exposure. Green arrows indicate the change in expression when the WNK/STK39/SPAK/NCC is activated. Blue arrows indicate downstream pathways. 


\section{References}

Arora, P., et al. (2011). "Genetic polymorphisms of innate immunity-related inflammatory pathways and their association with factors related to type 2 diabetes." Bmc Medical Genetics 12: 95.

Badawi, A., et al. (2010). "Type 2 diabetes mellitus and inflammation: Prospects for biomarkers of risk and nutritional intervention." Diabetes Metab Syndr Obes 3: 173-186.

Balvers, M. G., et al. (2010). "Docosahexaenoic acid and eicosapentaenoic acid are converted by 3T3-L1 adipocytes to $\mathrm{N}$-acyl ethanolamines with anti-inflammatory properties." Biochim Biophys Acta 1801(10): 1107-1114.

Barbu, A., et al. (2015). "The role of complement factor C3 in lipid metabolism." Mol Immunol 67(1): 101-107.

Bellin, M. D., et al. (2013). "Insulin secretion improves in cystic fibrosis following ivacaftor correction of CFTR: a small pilot study." Pediatr Diabetes 14(6): 417-421.

Berger, M., et al. (2002). "Structure and function of N-acetylglucosamine kinase Identification of two active site cysteines." European Journal of Biochemistry 269(17): 4212-4218.

Chatterjee, P., et al. (2013). "Adipocyte fetuin-A contributes to macrophage migration into adipose tissue and polarization of macrophages." J Biol Chem 288(39): 28324-28330.

Chiellini, C., et al. (2004). "Serum haptoglobin: a novel marker of adiposity in humans." J Clin Endocrinol Metab 89(6): 2678-2683.

Copeland, R. J., et al. (2008). "Cross-talk between GlcNAcylation and phosphorylation: roles in insulin resistance and glucose toxicity." American Journal of PhysiologyEndocrinology and Metabolism 295(1): E17-E28.

Cox, J. and M. Mann (2008). "MaxQuant enables high peptide identification rates, individualized p.p.b.-range mass accuracies and proteome-wide protein quantification." Nat Biotechnol 26(12): 1367-1372.

Cox, J., et al. (2011). "Andromeda: a peptide search engine integrated into the MaxQuant environment." Journal of Proteome Research 10(4): 1794-1805.

Dehwah, M. A. S., et al. (2012). "MicroRNAs and Type 2 Diabetes/Obesity." Journal of Genetics and Genomics 39(1): 11-18.

Derosa, G., et al. (2013). "Adipocytokine levels in obese and non-obese subjects: an observational study." Inflammation 36(4): 914-920.

Eriksson, J. W., et al. (2008). "Hydrochlorothiazide, but not Candesartan, Aggravates Insulin Resistance and Causes Visceral and Hepatic Fat Accumulation The Mechanisms for the Diabetes Preventing Effect of Candesartan (MEDICA) Study." Hypertension 52(6): 10301037. 


\section{Chapter 5}

Fang, L., et al. (2015). "Analysis of the Human Proteome in Subcutaneous and Visceral Fat Depots in Diabetic and Non-diabetic Patients with Morbid Obesity." J Proteomics Bioinform 8(6): 133-141.

Fullerton, M. D., et al. (2009). "The Development of a Metabolic Disease Phenotype in CTP: Phosphoethanolamine Cytidylyltransferase-deficient Mice." Journal of Biological Chemistry 284(38): 25704-25713.

Gagnon, K. B. and E. Delpire (2010). "On the substrate recognition and negative regulation of SPAK, a kinase modulating $\mathrm{Na}+-\mathrm{K}+-2 \mathrm{Cl}(-)$ cotransport activity." American Journal of Physiology-Cell Physiology 299(3): C614-C620.

Gupta-Ganguli, M., et al. (2011). "Does therapy with anti-TNF-alpha improve glucose tolerance and control in patients with type 2 diabetes?" Diabetes Care 34(7): e121.

Hirst, J. A., et al. (2015). "Quantifying the effects of diuretics and beta-adrenoceptor blockers on glycaemic control in diabetes mellitus a systematic review and meta-analysis." British Journal of Clinical Pharmacology 79(5): 733-743.

Hotamisligil, G. S., et al. (1995). "Increased Adipose-Tissue Expression of Tumor-NecrosisFactor-Alpha in Human Obesity and Insulin-Resistance." Journal of Clinical Investigation 95(5): 2409-2415.

Hotamisligil, G. S., et al. (1996). "IRS-1-mediated inhibition of insulin receptor tyrosine kinase activity in TNF-alpha- and obesity-induced insulin resistance." Science 271(5249): 665-668.

Karolina, D. S., et al. (2011). "MicroRNA 144 impairs insulin signaling by inhibiting the expression of insulin receptor substrate 1 in type 2 diabetes mellitus." PLoS One 6(8): e22839.

Keller, P., et al. (2011). "Gene-chip studies of adipogenesis-regulated microRNAs in mouse primary adipocytes and human obesity." BMC Endocr Disord 11: 7.

Klein, A. L., et al. (2009). "O-Linked N-Acetylglucosamine Modification of Insulin Receptor Substrate-1 Occurs in Close Proximity to Multiple SH2 Domain Binding Motifs." Molecular \& Cellular Proteomics 8(12): 2733-2745.

Kraus, D., et al. (2014). "Nicotinamide N-methyltransferase knockdown protects against dietinduced obesity." Nature 508(7495): 258-+.

Lisi, S., et al. (2011). "Obesity-associated hepatosteatosis and impairment of glucose homeostasis are attenuated by haptoglobin deficiency." Diabetes 60(10): 2496-2505.

Lo, J. C., et al. (2014). "Adipsin Is an Adipokine that Improves beta Cell Function in Diabetes." Cell 158(1): 41-53.

Lopez-Villar, E., et al. (2015). "A proteomic approach to obesity and type 2 diabetes." J Cell Mol Med 19(7): 1455-1470. 
Ma, J. H., et al. (2014). "Prolonged Insulin Stimulation Down-regulates GLUT4 through Oxidative Stress-mediated Retromer Inhibition by a Protein Kinase CK2-dependent Mechanism in 3T3-L1 Adipocytes." Journal of Biological Chemistry 289(1): 133-142.

Maffei, M., et al. (2016). "The Multifaceted Haptoglobin in the Context of Adipose Tissue and Metabolism." Endocr Rev 37(4): 403-416.

Mathur, S. K., et al. (2013). "Transcriptomic analysis of visceral adipose from healthy and diabetic obese subjects." Indian J Endocrinol Metab 17(3): 446-450.

Moreno-Navarrete, J. M., et al. (2010). "Complement factor H is expressed in adipose tissue in association with insulin resistance." Diabetes 59(1): 200-209.

Mori, S., et al. (2014). "Characteristic Expression of Extracellular Matrix in Subcutaneous Adipose Tissue Development and Adipogenesis; Comparison with Visceral Adipose Tissue." International Journal of Biological Sciences 10(8): 825-833.

Moriguchi, T., et al. (2005). "WNK1 regulates phosphorylation of cation-chloride-coupled cotransporters via the STE20-related kinases, SPAK and OSR1." Journal of Biological Chemistry 280(52): 42685-42693.

Myslicki, J. P., et al. (2014). "Role of O-GlcNAcylation in nutritional sensing, insulin resistance and in mediating the benefits of exercise." Applied Physiology Nutrition and Metabolism 39(11): 1205-1213.

Nakagawa, J., et al. (1995). "Auh, a Gene Encoding an Au-Specific Rna-Binding Protein with Intrinsic Enoyl-Coa Hydratase Activity." Proc Natl Acad Sci U S A 92(6): 2051-2055.

Nishida, H., et al. (2012). "Phosphatidylinositol 3-Kinase/Akt Signaling Pathway Activates the WNK-OSR1/SPAK-NCC Phosphorylation Cascade in Hyperinsulinemic db/db Mice." Hypertension 60(4): 981-+.

Pal, D., et al. (2012). "Fetuin-A acts as an endogenous ligand of TLR4 to promote lipidinduced insulin resistance." Nat Med 18(8): 1279-+.

Pavlovic, Z. and M. Bakovic (2013). "Regulation of Phosphatidylethanolamine HomeostasisThe Critical Role of CTP: Phosphoethanolamine Cytidylyltransferase (Pcyt2)." Int J Mol Sci 14(2): 2529-2550.

Quaye, I. K. (2008). "Haptoglobin, inflammation and disease." Trans R Soc Trop Med Hyg 102(8): 735-742.

Sabater, D., et al. (2013). "The use of Transwells improves the rates of differentiation and growth of cultured 3T3L1 cells." Anal Bioanal Chem 405(16): 5605-5610.

Scheja, L., et al. (2008). "Acute-Phase Serum Amyloid A as a Marker of Insulin Resistance in Mice." Experimental Diabetes Research.

Sjoholm, K., et al. (2005). "A Microarray search for genes predominantly expressed in human omental adipocytes: Adipose tissue as a major production site of serum amyloid A." Journal of Clinical Endocrinology \& Metabolism 90(4): 2233-2239. 


\section{Chapter 5}

Spiegelman, B. M., et al. (1993). "Regulation of Adipocyte Gene-Expression in Differentiation and Syndromes of Obesity Diabetes." Journal of Biological Chemistry 268(10): 6823-6826.

Starner, C. I., et al. (2008). "Rosiglitazone and pioglitazone utilization from January 2007 through May 2008 associated with five risk-warning events." Journal of Managed Care Pharmacy 14(6): 523-531.

van Greevenbroek, M. M., et al. (2012). "Up-regulation of the complement system in subcutaneous adipocytes from nonobese, hypertriglyceridemic subjects is associated with adipocyte insulin resistance." J Clin Endocrinol Metab 97(12): 4742-4752.

Vikman, P., et al. (2014). "RNA sequencing: current and prospective uses in metabolic research." Journal of Molecular Endocrinology 53(2): R93-R101.

Vlaicu, S. I., et al. (2016). "The role of complement system in adipose tissue-related inflammation." Immunol Res 64(3): 653-664.

Wajchenberg, B. L., et al. (2002). "Depot-specific hormonal characteristics of subcutaneous and visceral adipose tissue and their relation to the metabolic syndrome." Horm Metab Res 34(11-12): 616-621.

Weihofen, W. A., et al. (2006). "Structures of human N-acetylglucosamine kinase in two complexes with $\mathrm{N}$-acetylglucosamine and with ADP/glucose: Insights into substrate specificity and regulation." J Mol Biol 364(3): 388-399.

Weisberg, S. P., et al. (2003). "Obesity is associated with macrophage accumulation in adipose tissue." Journal of Clinical Investigation 112(12): 1796-1808.

Whelan, S. A., et al. (2010). "Regulation of Insulin Receptor Substrate 1 (IRS-1)/AKT Kinase-mediated Insulin Signaling by O-Linked beta-N-Acetylglucosamine in 3T3-L1 Adipocytes." Journal of Biological Chemistry 285(8): 5204-5211.

Wlazlo, N., et al. (2014). "Complement factor 3 is associated with insulin resistance and with incident type 2 diabetes over a 7 -year follow-up period: the CODAM Study." Diabetes Care 37(7): 1900-1909.

Xita, N. and A. Tsatsoulis (2012). "Adiponectin in Diabetes Mellitus." Current Medicinal Chemistry 19(32): 5451-5458.

Yamashita, A., et al. (2007). "Macrophage-adipocyte interaction: marked interleukin-6 production by lipopolysaccharide." Obesity (Silver Spring) 15(11): 2549-2552.

Yang, D. K., et al. (2011). "IRBIT governs epithelial secretion in mice by antagonizing the WNK/SPAK kinase pathway." Journal of Clinical Investigation 121(3): 956-965.

Yang, R. Z., et al. (2006). "Acute-phase serum amyloid A: An inflammatory adipokine and potential link between obesity and its metabolic complications." Plos Medicine 3(6): 884894.

Zhao, X., et al. (2012). "Silencing SOCS3 could inhibit TNF-alpha induced apoptosis in 3T3L1 and mouse preadipocytes." Molecular Biology Reports 39(9): 8853-8860. 


\section{Supplementary figures}

\begin{tabular}{|l|l|}
\hline mNAGK F1 & CACGGTCCAAAGTCCTTTTACT \\
\hline mNAGK R1 & GTCTGTGCCAATCAGCCAGT \\
\hline mNAGK F2 & GCTAGGGTCGAGGTTGCTAC \\
\hline mNAGK R2 & AGTAAAAGGACTTTGGACCGTG \\
\hline mAUH F1 & AGCTGGCTCTAGCGTGTGA \\
\hline mAUH R1 & GTTCTGTTCTAACACATGGCTGA \\
\hline mAUH F2 & AATTTGCACCTTTTTGGTGGC \\
\hline mAUH R2 & AGTCAGGAGTCACTTCTGGCT \\
\hline mNNMT F1 & TGTGCAGAAACGAGATCCTC \\
\hline mNNMT R1 & AGTTCTCCTTTTACAGCACCCA \\
\hline mNNMT F2 & GCCCCACCATCTATCAGCTTC \\
\hline mNNMT R2 & ACGCCTCAACTTCTCCTCCT \\
\hline m pCYT2 F1 & CGATGGCTGCTATGACATGGT \\
\hline m pCYT2 R1 & GCCCCTTATGCTTGGCAATCT \\
\hline mSTK39 F1 & TCAGGCAGCCCTATGCAAAC \\
\hline mSTK39 R1 & TGGCTCATGGCTTGAATTTCTT \\
\hline mSTK39 F2 & TTCAGGCAGCCCTATGCAAAC \\
\hline mSTK39 R2 & TGGCTCATGGCTTGAATTCTT \\
\hline mIL1a F1 & CGAAGACTACAGTTCTGCCATT \\
\hline mIL1a R1 & GACGTTTCAGAGGTTCTCAGAG \\
\hline mIL1b F1 & GCAACTGTTCCTGAACTCAACT \\
\hline mIL1b R1 & ATCTTTTGGGGTCCGTCAACT \\
\hline mCsnk2b F1 & AATGAGCAGGTGCCTCACTAT \\
\hline mCsnk2b R1 & TGTTCGATCAAGTCGCTCTGG \\
\hline mCsnk2a1 F1 & ATGTGGTGGAATGGGGGAATC \\
\hline mCsnk2a1 R1 & GCAAGTGTGATGATGTTGGGC \\
\hline mCsnk2a1 F2 & AGGATAGCCAAGGTTCTGGGA \\
\hline mCsnk2a1 R2 & CCATCGCTTACGGGAGTGTC \\
\hline C3-FOR & AGCAGGTCATCAAGTCAGGC \\
\hline C3-REV & GATGTAGCTGGTGTTGGGCT \\
\hline CFB-FOR & CTCCTCTGGAGGTGTGAGCG \\
\hline CFB-REV & GGTCGTGGGCAGCGTATTG \\
\hline Adipsin-FOR & TCAATCATGAACCGGACAAC \\
\hline Adipsin-REV & ATGCCTGCCATCCAACCTG \\
\hline Adiponectin F & \\
\hline Adiponectin R & \\
\hline
\end{tabular}

Figure S1. QPCR primers. 


\section{Chapter 5}

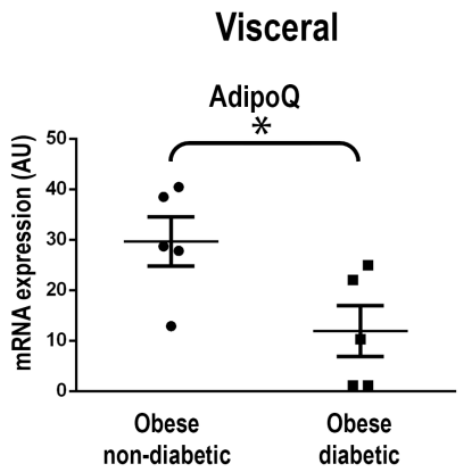

Subcutaneous
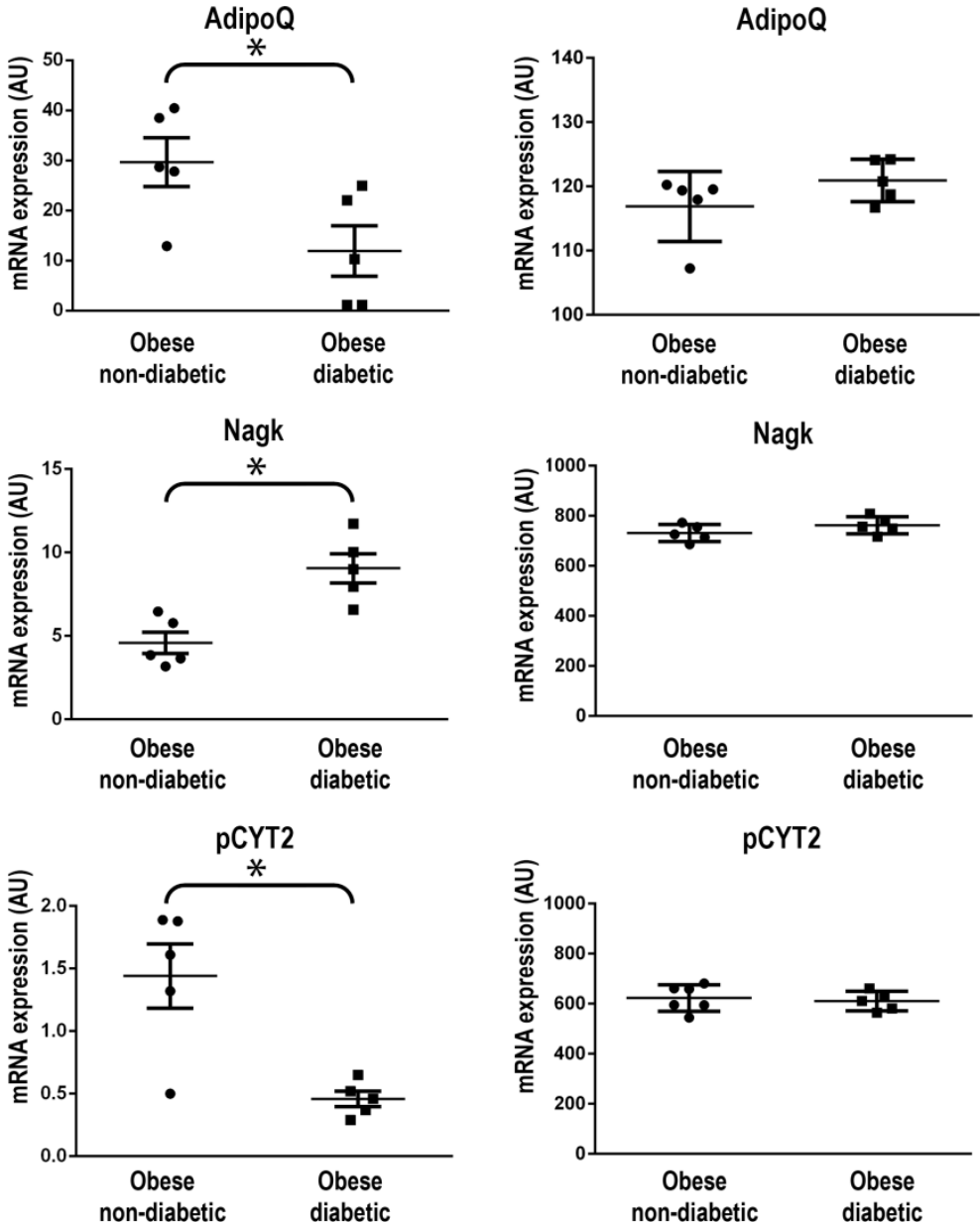

pCYT2
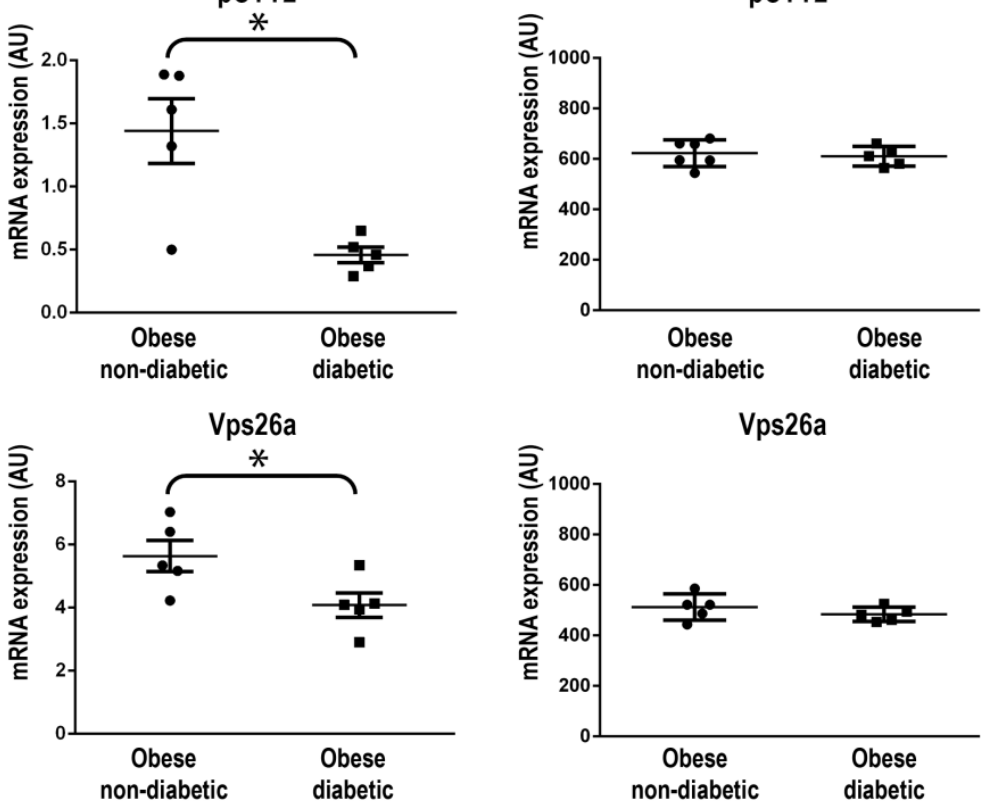

Figure S2. mRNA expression in visceral and subcutaneous sample of obese non-diabetic and obese diabetic subjects. We analyzed the mRNA expression, of genes identified through proteomics, in visceral and subcutaneous adipose tissue of healthy obese versus T2DM obese from published data sets. Every data point represents 1 subject. * indicates p-values which are lower than 0.05 with non-parametric t-test according to Mann-Whitney. 




\section{Chapter 6}

\section{Discussion and future perspectives}




\section{Chapter 6}

\section{Discussion}

One of the most frequent diseases related to lifestyle and obesity is type 2 diabetes mellitus (T2DM). Diabetes results when the body is unable to produce sufficient amounts of insulin or does not respond properly to insulin. Insulin is the hormone that regulates the level of glucose in blood (Saltiel et al., 2001). The increase of glucose in the blood in T2DM may cause hyperglycemia that leads to long term complications.

T2DM is the most common type of diabetes and is a result of impaired activity of $\beta$-cells, which are the cells that produce insulin, in combination with the reduced insulin sensitivity of the insulin-targeted organs. T2DM is closely related with the metabolic syndrome which is described by abdominal obesity, atherogenic dyslipidemia, elevated blood pressure, insulin resistance and prothrombotic and proinflammatory states (Tenenbaum et al., 2003). Obesity is also characterized by "low grade" chronic inflammation (Monterio et al., 2010), or as metabolically triggered inflammation (metaflammation) (Hotamisligil, 2006) or parainflammation, meaning the state between normal and inflammation states (Medzhitov, 2008). Until now, the treatments for diabetes did not have a preventive character for the complications associated with diabetes, such as neuropathy, retinopathy and nephropathy (Segev et al., 2003). Usually treatments focused on the use of exogenous insulin to control the level of glucose or on the transplantation of the whole-pancreas organ which has been the most effective treatment for diabetic people with severe complications, until now (BonnerWeir et al., 2005). One of the major drawbacks of this approach is the limited supply of donor tissues (Weir et al., 1997). Until recently one of the focus points for medication for T2DM was on the use of peroxisome proliferator-activated receptor gamma (PPAR $\gamma$ ) agonists. Specific ligands for PPAR $\gamma$ are thiazolidinediones (TZDs) (Berger et al., 2002; Yki-Jarvinen, 2004). However, only pioglitazone has remained in use after troglitazone and rosiglitazone were withdrawn from the market because of hepatotoxicity and increased risk of heart attacks, respectively. In some countries however even pioglitazone has been withdrawn because of its possible association with increased incidence of bladder tumors. Recently new strategies, such as the GLP-1 agonists and DPP-4 inhibitors started being established as potential T2DM therapies and they start to be recognized by the American and European Diabetes Associations (Nauck 2016).

T2DM is a systemic disease which makes it difficult to identify and establish proper targets for the treatment or cure of the patients. Until recently research was focused on the main 


\section{Discussion and future perspectives}

target organs such as the pancreas and the skeletal muscle. Nowadays, after the adipose tissue has been characterized as an organ, research focuses on adipocytes and their role in the disease (Galic et al., 2010).

The aim of this project was to define novel targets for the possible development of drugs for T2DM. Optimization of present preventive therapies including development of less expensive drugs may be of help. In the present thesisa search for novel biomarkers and drug targets for T2DM was undertaken.

Chapter 1 of the thesis provided an introduction to the topic and an overview of T2DM and the mechanisms behind it. T2DM results from impaired insulin production by the beta-cells of the pancreas and from the inability of the peripheral organs targeted by insulin to exert adequate glucose uptake from the blood leading to insulin resistance. In this thesis we focused especially on possible new drug strategies and novel drug targets that deal with insulin resistance.

In chapter 2 we focused on another possible target for insulin therapy in addition to PPAR $\gamma$ and investigated whether resistin downregulation in mouse adipocytes would provide an alternative for PPAR $\gamma$ agonist activity when testing new diabetic drugs. The results obtained revealed significant correlations between the $\mathrm{EC}_{50}$ for PPAR $\gamma$ activation in a reporter gene cell line and resistin downregulation in mouse adipocytes, and between the $\mathrm{IC}_{50}$ for resistin downregulation and the minimum effective dose for antihyperglycemic activity in a mouse model. These correlations indicate that PPAR $\gamma$ mediated downregulation of resistin might promote insulin sensitivity and that downregulation of resistin in mouse adipocytes provides an adequate bioassay for screening of newly developed antihyperglycemic compounds. These findings can assist in the finding of insulin sensitizing compounds by using resistin as a target. Resistin is a hormone that is involved in inflammation and insulin resistance (Saltiel et al., 2001; Steppan et al., 2005; Lazar et al., 2007; Owecki et al., 2011). Resistin mRNA is mainly found in adipose tissue in mouse and in adipose tissue and macrophages in human. On the other hand resistin is found in blood which means that resistin can be distributed over and affect the whole body. The most important is that resistin is a link of obesity to T2DM since elevated levels of resistin were found in diabetes patients (Hartman et al., 2002; Lazar, 2007; Rajala et al., 2004; Stofkova et al., 2010). Exactly this link makes resistin an interesting possible novel target in this field of studies. Interesting is the fact that until now the actual receptor of resistin is not known. Few speculations by different research groups suggested 


\section{Chapter 6}

different receptors but this has to be further confirmed. One of the proposals is the involvement of the Toll-like receptor 4 (TLR-4) and this was investigated in more detail in Chapter 3 since it would provide an interesting novel target for development of new drugs for 2TDM.

In chapter 3, we investigated if a known commercially available assay for TLR-4 binding can be used for detecting compounds that could assist in defining novel drugs for insulin sensitivity (Tarkowski et al., 2010). TLR-4 is a receptor known to be involved in inflammation and to be correlated with insulin sensitivity (Tarkowski et al., 2010). In Chapter 3 we investigated whether a reporter assay detecting TRL-4 mediated gene expression could be a possible bioassay to detect candidate drugs able to inhibit resistin mediated TLR-4 activation and subsequent effects. If so, inhibition of this resistin mediated activity may prove a novel target for development of new generations of antidiabetic drugs. To detect activation of TLR-4 mediated gene expression and enable detection of possible antagonists for resistin mediated activation of the receptor, a TLR-4 reporter gene cell line (HEK-Blue-TLR-4) was applied. The results obtained in chapter 3 reveal that resistin, like the natural TLR-4 ligand lipopolysaccharide (LPS) is able to activate TLR-4 mediated gene expression with $\mathrm{EC}_{50}$ values for LPS and resistin amounting to respectively $0.05 \mu \mathrm{g} / \mathrm{ml}$ and $5 \mu \mathrm{g} / \mathrm{ml}$. Resistin was also shown to compete with LPS for binding to TLR-4 in a human epithelial cell line and to promote overall inflammation (Tarkowski et al., 2010; Benomar et al., 2012). The LPS or resistin dependent induction of TLR-4 mediated gene expression was inhibited by two known TLR-4 inhibitors including LPS-RS (Visintin et al., 2005) and Polymyxin B (Duff et al., 1982). It is concluded that the TLR-4 reporter gene cell line provides a novel tool to detect resistin antagonists and may be of use as a new target to screen for a possible new type of antidiabetic drugs.

The importance of resistin in insulin sensitivity and T2DM has been established by several researchers. In chapters 2 and 3 of the thesis we investigated methods that include resistin and would help the identification of compounds interfering with the possible role of resistin in impairment of insulin sensitivity. To further increase the possible identification of novel targets for antidiabetic drugs via the possible role of resistin we focused in chapter 4 on the mechanism behind resistin mediated effects by exposing adipocytes to resistin and analyzing the effects on genes expression. Thus we identified genes in adipocytes that were up or down regulated upon their exposure to resistin. Overall the results showed that resistin upregulates or downregulates a large set of genes in the adipocytes. The protein products of the genes up- 


\section{Discussion and future perspectives}

or downregulated are located mostly in the nucleus but also in the cell membrane, in the cytoplasm and in the mitochondria. It was also observed that several miRNAs were influenced in the presence of resistin. From the gene expression data it can be observed that resistin is involved in the downscaling of cellular maintenance and cellular processes. Resistin is in addition involved in lipid storage. From these observations we propose that resistin is expressed when energy conservation is essential. When energy conservation is accompanied by food overconsumption, obesity, inflammation and insulin resistance can be the consequence. Resistin can therefore be considered as a biomarker protein for these health related issues.

In chapter 5 we continued the search for novel targets for T2DM drugs using an in vitro model that mimics inflamed adipocytes by combining 3T3-L1 adipocytes and activated RAW264.7 macrophages, producing an environment for testing compounds under conditions more similar to the in vivo situation in T2DM patients. Using this cell model we identified new proteins and we confirmed these proteins by literature data-mining on human mRNA expression of visceral fat of obese healthy versus obese T2DM subjects. These proteins have the potency to be used as biomarkers for T2DM progression. Furthermore, the observed biomarkers are connected to pathways that will assist to describe the development of T2DM and consequently to design more specific therapeutic approaches. Importantly, our results also indicate that the in vitro approaches we have undertaken are translational to the human situation.

The co-cultures of adipocytes and macrophages have expression profiles that are comparable to visceral adipose tissues and, as such, are very interesting as an in vitro model for drug screening and research on signaling pathways involved in insulin sensitivity. In addition, we identified proteins that are known factors in the development of atherosclerosis (Kraus et al., 2014; Yang et al., 2006), indicating that this in vitro model can also help to clarify the role of inflamed adipose tissue in multiple other aspects of the metabolic syndrome and it is not restricted to diabetes.

Al together it is concluded that the contact co-culture of 3T3-L1 with activated RAW264.7 is a mechanistically relevant and translational representative of inflamed visceral adipose tissue, which links to development of T2DM and can be used in identifying biomarkers of T2DM regulation. 


\section{Chapter 6}

\section{Future perspectives}

As mentioned above T2DM results in insufficient insulin production and impaired insulin uptake by the targeted peripheral organs (insulin resistance). In this thesis we focused mainly on searching for novel targets that can be of use in defining new strategies and methods to overcome insulin resistance. Based on the results presented here and on other scientific literature additional strategies can be formulated, focusing not only on insulin resistance but also on insulin production. Some future perspectives, directions and suggestions that follow from this work and the results obtained in the present thesis are discussed in the following sections.

\section{Future perspective: Stem cell based models}

In the present thesis adipocytes as well as inflamed adipocytes consisting of cocultures of 3T3-L1adipocytes and RAW264.7 macrophages were used to define novel models for defining drugs for T2DM. Novel stem cell based approaches will provide even more challenging and promising approaches for the future. Human embryonic stem cells are pluripotent and self-renewing cells derived from the inner cell mass of a fertilized egg, which has been cultured in vitro to reach the blastocyst stage (5-7 days after fertilization) (Thomson et al., 1998). Their inherent abilities (i) to indefinitely proliferate in the undifferentiated form, (ii) to differentiate in all three germ layers, under appropriate culture conditions, and (iii) the fact that they are cells of human origin, makes them very useful in regenerative medicine and drug safety assessments. More than $400 \mathrm{hESC}$ lines are available, and some of them are well characterized and eligible for commercial use. These are organized in international stem cell banks, such as the National stem cell bank (www.nationalstemcellbank.org), the UK stem cell bank (www.ukstemcellbank.org.uk), the hESCreg (www.hescreg.eu) (Jensen et al., 2009).

The need to provide cell-based assays that are physiologically relevant to human, without these cells losing their functional properties after some time, and that are relatively easy to access, is really important for drug discovery and risk assessment. These properties are all gathered in hESCs. They provide a source of virtually unlimited cells that are physiologically relevant to human cells, which could lower the cost-effective assays leading to lower attrition rates and safer drugs by increasing the predictability of the assays (Sartipy et al., 2007). These hESCs should be considered to develop into models that facilitate studies on novel targets for T2DM therapy, including for example pancreatic lineage and insulin producing $\beta$-cells 


\section{Discussion and future perspectives}

There is a number of methods that have been developed for the differentiation of hESC to pancreatic lineage (Kroon et al., 2007; Jiang et al., 2007; D’Amour et al., 2006; Chen et al., 2009). Due to the difficulties in differentiation, most of the methods are practically insufficient, since the final population of $\beta$-cells is really low comparison with the total cell population cultured in the beginning. A highly relevant protocol was described by D'Amour et al. (2006), taking into consideration the pathway of the pancreas organogenesis. It is a five stage process from hESC to $\beta$-cell aggregates. The final result is insulin producing cells.

The five different stages of the differentiation process of the hESC to $\beta$-cell, aggregates can be described as follows (D'Amour et al., 2006):

Stage 1: Definitive endoderm (DE): Critical for the direction to pancreatic lineage is the differentiation of the human embryonic stem cells into definitive endoderm. This is because the differentiation of stem cells is nonreversible under normal cultures conditions (Guo et al., 2009). Thus the differentiation must be efficient in the sense of the percentage of cells differentiated compared with the cells differentiated in a developing embryo. The differentiation in this first stage is achieved by exposing the undifferentiated hESCs to activin A $(100 \mathrm{ng} / \mathrm{ml})$ for 3 days. Wnt3a $(25 \mathrm{ng} / \mathrm{ml})$ is added during the first day of activin A exposure. The differentiation should approximately reach $80 \%$ of the initial cell population. The cells at this point should express definitive endoderm's markers SOX17 and CXCR4 (D’Amour et al., 2005).

Stage 2: Primitive gut (PG): To reach stage 2, activin A is removed from the cells. This allows the cells to differentiate to primitive gut tube. By adding at this point also FGF10 (fibroblast growth factor 10) and a Hedgehog inhibitor, KAAD-cyclopamine, for 2-4 days, INS mRNA will increase at a later stage (stage 5). At this point gut tube markers HNF1B and HNF4A should be expressed along with SOX17. On the other hand definitive endoderm markers CER and CXCR4 should be reduced.

Stage 3: Posterior foregut (PF): After 4 days the cells proceed to stage 3. Along with FGF10 and KAAD-cyclopamine cells are exposed to retinoic acid (RA) for 2-4 days. By this time cells are directed to posterior foregut. RA has been proven to be crucial for the direction to pancreatic lineage (Chen et al., 2004). High levels of PDX1 and HNF6 markers should be expressed at this point. Parallel an increase of HNF1B and HNF4A should also be observed. The differentiation at this point reaches $50 \%$ of the initially present hESCs. 


\section{Chapter 6}

Stage 4: Pancreatic endoderm (PE): The next stage of the differentiation, stage 4, is achieved by the removal of RA. With the removal of RA, after 3 days, the cells differentiate from posterior foregut to pancreatic endoderm and endocrine precursor. Co-expression of PDX1 and NKX1 should be observed, at this point, which represents pancreatic epithelium. In addition, many NKZ2-2 expressing cells should co-express NGN3, reflecting initial commitment to the endocrine lineage. At this point of differentiation the differentiated cells amount to $35 \%$ of the original population.

Stage 5: Langerhans islets, that contain $\beta$-cells: The last and final stage according to D'Amour et al. (2006) is achieved by adding growth factors, such as Ex4, IGF1 and HGH. By leaving the cells for at least 3 days to mature, expression of PDX1 and NKX61 should be observed. The final differentiated cells (representing $\beta$-cells in islets-like aggregates) amount to only a relatively low percentage $(7 \%)$ of the total cell population. These cells respond to multiple secretory stimuli but not to glucose, which is also a characteristic of the fetal pancreas, indicating that they are immature (D'Amour et al., 2006).

An additional study from Kroon et al. (2007) described a procedure in which the final differentiation step in this process was carried out in vivo and resulted in the formation of glucose responsive cells with insulin secretory properties similar in kinetics and magnitude to similarly engrafted adult human islets. This was achieved by a direct engraftment of cell aggregates of the pancreatic endoderm (stage 4) into immunosufficient mice and generated glucose-responsive, insulin-secreting cells providing a very promising cell-replacement therapy.

An alternative option at stage 4 is to maintain RA and transfer cells to modified islet maturation medium as described by Jiang et al. 2007. In this modified protocol, bFGF (10 $\mathrm{ng} / \mathrm{ml})$ was added the first 3 days and nicotinamide $(10 \mathrm{nM})$ for the next 5 (Jiang et al., 2007). By this, maturation of the cells that are already committed (stage 4) to pancreatic lineage is achieved. Using this modified protocol the percentage of hESC differentiated to the final stage of differentiation might reach $15 \%$ and these cells might even respond to variations in glucose levels.

It is also worth noting that in the method of Chen et al. (2009) they have identified a small molecule, (-)-indolactam V (ILV), that induces differentiation of hESCs to a substantial number of Pdx-1 expressing cells. This population could release c-peptide following glucose stimulation. The ILV has been proven to work specifically at one stage of pancreatic 


\section{Discussion and future perspectives}

development. It directs the pancreatic specification of hESCs that have already committed to the endoderm lineage; that is the gut tube endoderm (stage 3 of the method of D' Amour et al., 2006). According to Chen et al. (2009) one of the remaining challenges is to find molecules inducing differentiation to pancreatic lineage when added at each stage and also to determine which stage is optimal for transplantation. A suggestion is to aim at identification of new chemical compounds to optimize the differentiation process.

More recent studies pointed in this direction. Several groups studied and/or used insulin producing $\beta$-cells differentiated from hESC to find new genes involved, characterize novel compounds that can assist the differentiation or optimizing the existing methods. Schaffer et al. (2013) found a gene NKx6.1 (NK6 homeobox 1) that regulates a network responsible for pancreatic $\beta$-cell identity. Likewise Cai et al. (2013) showed that NGN-3 expressing progenitors assist in the generation of pancreatic endocrine cells, and Cage et al. (2015) showed that ARX (Aristaless Related Homeobox) is a major factor in the specification of somatostatin, glucagon and insulin in cells derived from hESCs. Resveratrol is one of the compounds that it was shown to improve the maturation of $\beta$-cells derived from hESCs (Pezolla et al., 2015). Also several studies have demonstrated optimization and improvement in the steps needed in achieving insulin producing $\beta$-cells (Wang et al., 2015; Bose et al., 2012; Richardson et al., 2015),

Another advantage of stem cell technology is that by understanding the function of stem cells, we understand how organisms develop and grow and how tissues are maintained through life. A significant knowledge to understand diseases and injuries and how to treat them is revealed with the understanding of stem cells. Therefore stem cell technology provides a new tool for better understanding the mechanisms involved in drug-induced adverse reactions and to potentially predict and avoid toxicity in humans (Davila et al., 2004).

\section{Future perspective: elucidating the role of resistin in insulin sensitivity.}

Another aspect that deserves attention in future developments is the further elucidation of the role of resistin in T2DM. Resistin plays an important role in insulin resistance and in inflammation (Mojiminiyi et al., 2007; McTernan et al., 2006; Holcomb et al., 2000; Reilly et al., 2005; Fasshauer and Blüher, 2015). Several mechanisms have been proposed for resistin's activity such as inhibition of cell surface glucose transporters, activation of suppressors of cytokine signaling (SOCS-3), blocking of insulin signaling pathways, stimulation of hepatic 


\section{Chapter 6}

glucose production and activation of free fatty acids release from adipose tissue (Moon et al., 2003; Steppan et al., 2005; Brown et al., 2007; Sheng et al., 2008; Banerjee et al., 2004; Pravenec et al., 2006; Stofkova, 2010; Fasshauer and Blüher, 2015; Al Hannan and Culligan, 2015). Additionally resistin promotes overall inflammation through TLR-4 (Benomar et al., 2012). It is obvious that prevention of resistin activity would improve insulin sensitivity (Lazar, 2007; Blüher, 2014). Reduction of resistin's expression may possibly contribute to T2DM treatment, and may even not be restricted to treatment of the adverse health effects of T2DM. Testing and finding inhibitors or antagonists of resistin from known chemical data bases using the methods described in this thesis may prove to be a way forward. Additionally blockage of resistin function might be possible with the use of anti-resistin antibodies from immunized animals as Steppan and his colleagues showed before (2001) that administration of anti-resistin antibodies improved blood sugar and insulin action in mice with diet-induced obesity. To further elucidate the role of resistin in T2DM the actual nature and role of the receptor of resistin must be further elucidated (Daquinag et al., 2011; Al Hannan and Culligan, 2015) since only putative receptors have been reported such as CAP-1 (Lee et al., 2014), an isoform of decorin (Daquinag et al., 2011), mouse receptor tyrosine kinase-like orphan receptor 1 (ROR1) (Sanchez-Solana et al., 2012) and Toll-like receptor 4 (Tarkowski et al., 2010). The identification of the actual receptor will reveal the pathophysiological role of resistin which in turn will contribute to the development of novel diagnostic tools (Park et al., 2013). Considering that resistin protein is found to circulate in the blood and can affect multiple tissues and organs a possible way forward would be to perform RNA sequencing in samples from all the affected organs from diabetic patients and compare the genes that are regulated with their expression levels in non-diabetic patients. This extended comparison might shed light on the actual mechanism behind resistin mediated effects in T2DM and reveal possible biomarkers that could be resistin's receptor or could be used as potential novel pharmacological targets. All the above possible findings have to be translated into human subjects considering the fact that human and mouse resistin are not homologous (Al Hannan and Culligan, 2015; Park et al., 2013; Ghosh et al., 2003; Patel et al., 2004).

\section{Future perspective: Organokines}

It has been mentioned before that adipose tissue acts as an endocrine organ secreting cytokines called adipokines that regulate systemic metabolism and energy homeostasis (Galic et al., 2009; Molica et al., 2014; Choi, 2015). Similarly, studies have shown that other organs secret proteins that affect whole body metabolism through autocrine, paracrine and endocrine 


\section{Discussion and future perspectives}

activity (Choi, 2015; Jung et al., 2016) and they are termed in line with the organ from which they are secreted. Skeletal muscles secrete myokines and liver secretes hepatokines (Febbraio and Pedersen, 2005; Stefan and Haring, 2013).

Skeletal muscle is considered to be the largest organ, composing $40 \%$ of body weight and not only contributing to energy storage but also to the depletion of energy (Eckardt et al., 2014; Roden, 2015; Samuel and Shulman, 2012). Skeletal muscle is now characterized as an endocrine organ (Febbraio et al., 2005; Pratesi et al., 2013). Hundreds of proteins have been identified to be secreted by skeletal muscle but more recently 50 novel myokines have been identified that cross-talk with other tissues such as adipose tissue, liver and pancreas and their regulation may improve insulin resistance (Eckardt et al., 2014; Raschke et al., 2013; Hartwig et al., 2013; Norheim et al., 2011; Gamas et al., 2015). Skeletal muscle's secretion of myokines depends on their contractile activity and this may be proved to be the link of physical exercise and health promoting effects of exercise (Eckardt et al., 2014). Also physical inactivity is shown that is a risk factor for a number of diseases including T2DM and cardiovascular disease (Tuomilehto et al., 2001; Nocon et al., 2008; Pedersen, 2010; Ertek and Cicero, 2012). Here it is mentioned one example of these myokines which is interleukin-6 (IL-6) (Ahima and Park, 2015; Eckardt et al., 2014). IL-6 was the myokine that was found to be secreted first in the blood upon muscle contraction and is also known as proinflammatory cytokine (Choi, 2015). IL-6 is secreted mainly by macrophages but during exercise, is also secreted by myofibres (Hiscock et al., 2004). IL-6 improves insulin sensitivity during acute exercise but induces insulin resistance in adipose tissue and in liver when its levels are chronically elevated (Catoire and Kersten, 2015). When secreted by macrophages then its signaling is regulated by the NF-kB pathway but when it is secreted by the myofibres then it is regulated mainly by $\mathrm{Ca} 2+/$ nuclear factor of activated T-cells (NFAT) and glycogen/p38 mitogen-activated protein kinase (MAPK) pathways (Brandt and Pedersen, 2010, Pedersen, 2010). The ratio of muscle to immune cell is considered to be responsible for the antiinflammatory or proinflammatory properties of IL-6 (Pedersen and Fabbraio, 2008, Pedersen, 2010). When the balance is on the antiflammatory side then IL-6 has a beneficial role in insulin sensitivity and glucose homeostasis (Pedersen and Fabbraio, 2007).

Comparable to proteins secreted form skeletal muscles, liver also secretes proteins, hepatokines, that regulate whole body homeostasis through the regulation of glucose and lipid metabolism and cross-talking with other tissues (Stefan and Haring, 2013; Iroz et al., 2015). Yoo et al. have shown that hepatokines are a link between obesity and cardiovascular 


\section{Chapter 6}

diseases. Two examples of such hepatokines are Fetuin-A and Fibroblast growth factor 21 (FGF21). Fetuin-A proved to play an important role in metabolic disease (Iroz et al., 2015). Elevated levels of Fetuin-A were observed in obese subjects and are correlated with insulin resistance and glucose intolerance through the inhibition of tyrosine kinase activity of the insulin receptor and the inhibition of GLUT4 (Iroz et al., 2015; Stefan and Haring, 2013; Takata et al., 2009). Additionally Fetuin-A may induce inflammation and insulin resistance as it is characterized as an adaptor protein for saturated fatty acid mediated TLR-4 activity (Pal et al., 2013; Mukhopadhyay and Bhattacharya, 2016). Consequently, Fetuin-A is contributing to the development of T2DM (Heinrichsdorff et al., 2012; Andersen et al., 2008; Mathews et al., 2002). On the other hand FGF21 proved to be beneficial to glucose metabolism and lipid homeostasis (Iroz et al., 2015). FGF21 has the ability to induce rapid loss of weight in rodents and to reduce blood glucose levels by stimulating glucose uptake in human primary adipocytes without the help of insulin (Kharitonenkov and Adams 2014). The beneficial effects of FGF21 have been translated to non-human primates and obese patients with T2DM which makes it a promising pharmacological target (Iroz et al., 2015; Gimeno et al., 2014).

These recent discoveries, recognitions and characterizations of novel organokines seem to provide a proof and a motive to continue unravelling the secretome of such complex diseases. And in order to decipher the secretome and identify novel biomarkers that can be used as pharmacological targets new robust and unequivocal methods have to be developed (Park et al., 2015, Bajaj and DeFrozo, 2003).

\section{Future perspective: Incretin effect}

The incretin effect is defined as the increase in glucose-dependent insulin secretion in response to nutrients, in comparison to glucose infused intravenously (Holst and Orskov 2004; Nauck 2016; Lutz and Osto 2016). In T2DM the incretin effect is decreased (Nauck 2016). Incretin hormones such as the gastrointestinal peptide hormone glucagon-like peptide 1 (GLP-1) bind to GLP-1 receptor and promote the release of insulin secretion from pancreatic $\beta$-cells (de Graaf et al. 2016). Additionally GLP-1 has been shown to play a role in different models that promote $\beta$-cell proliferation, neogenesis and inhibit apoptosis (Buteau 2011; Perfetti et al. 2011; de Graaf et al. 2016). Therefore finding agonists for the GLP-1 receptor can be a promising tool to define novel drug targets for T2DM. However the half life of GLP-1 is very low ( $2 \mathrm{~min}$ ) because of degradation by dipeptidyl peptidase-4 (DPP-4) (Drucker and Nauck 2006; Nauck 2016). Thus the therapeutic strategies so far focus on either 
extending the half life of GLP-1 by reducing its degradation via inhibitors of DPP-4 or defining GLP-1 agonists with longer half lives. Progress has been made in this field and the first GLP-1 agonist discovered, exenatide, has a half life of $2.4 \mathrm{~h}$ while the latest agonist which is albiglutide has a half life of 5 days. Furthermore DPP-4 inhibitors have been demonstrated to reduce DPP-4 activity maintaining elevated levels of GLP-1 concentrations (Ahren et al. 2004, Nauck 2016). Several selective inhibitors have been reported until now such as sitagliptin, saxagliptin, linagliptin and alogliptin (Nauck 2016).

\section{Future perspective: SGLT-2 inhibitors}

Another strategy that is recently used for the treatment of T2DM is the inhibition of sodiumglucose transporter-2 (SGLT-2). SGLT-2 is responsible for almost 90\% of the reabsorption of glucose in the blood after it is filtered into the pro-urine in the kidney (Schwartz and Ahmed 2016; DeFrozo et al. 2012). Increased expression and activity of SGLT-2 is observed in T2DM patients (Schwartz et al. 2016). Increased expression of SGLT-2 results in increased reabsorption of glucose and makes that glucose excretion in the urine occurs only when plasma glucose levels are high, sustaining in this way hyperglycemia (DeFrozo et al. 2013; Schwartz et al. 2016). The role of SGLT-2 inhibitors is to decrease glucose reabsorption leading to excretion of glucose in the urine, thus facilitating lowering glucose plasma levels (Reed 2016; Scheen 2016; Jabbour et al. 2012; DeFrozo et al. 2012; Schwartz et al. 2016). Examples of SGLT-2 inhibitors are canagliflozin, dapaglifloxin and empagliflozin.

\section{Future perspective: Cholesterol arachidonate ester}

According to our findings from RNA sequencing of human adipocytes exposed to resistin (chapter 4), c11orf10 gene is up regulated. This gene, according to Tanaka et al. (2008) is associated with the FADS (fatty acid desaturate) genes, which in turn are associated with elevated levels of polyunsaturated fatty acids (PUFAs) and arachidonic acid (Guan et al., 2015). The increase of cholesteryl esters is correlated with insulin resistance (Melone et al., 2012). Based on the results of our pilot experiments it can be speculated that this cholesterol ester is cholesterol arachidonate ester. Therefore a suggestion is that regulation of cholesterol arachidonate ester by resistin is another mechanism of resistin towards insulin resistance and T2DM. Also from pilot experiments reported in the present thesis, in which gene expression was analysed in adipocytes exposed to resistin in combination with cholesterol ester arachidonate possible target genes that showed up or down regulation were identified such as Ribosomal Protein L11 (RPL11), Rho-associated Protein Kinase 2 (ROCK2), Integrinalpha-6 


\section{Chapter 6}

(ITGA6), Acidic Ribosomal Phosphoprotein P0 (RPLP0) and Basic Transcription Factor 3 (BTF-3). These genes were regulated to a high extent but not significantly, thus giving us only indications and not solid proof for their resistin mediated regulation. Further investigation is needed about these new possible targets to verify their role in insulin sensitivity.

\section{Conclusions}

In this thesis new therapeutic targets for treatment and prevention of T2DM were investigated and identified. The fact that T2DM is a systemic disease makes it hard to identify specific targets and makes identification of novel drug targets a complex problem. Nevertheless the approaches described and results obtained pointed at some new targets that can be used in the future to identify new drug development strategies. There is sincere hope that the models developed in the present thesis will contribute to the definition of novel therapeutic strategies and enable answering some questions about a disease that has been studied for a very long period, although at the same time it is acknowledged that there are a lot of remaining questions for a complex endpoint.

\section{References}

Ahren, B., et al. (2004) "Inhibition of dipeptidyl peptidase-4 reduces glycemia, sustains insulin levels, and reduces glucagon levels in type 2 diabetes." J Clin Endocrinol Metab 89: 2078-2084.

Al Hannan, F. and K. G. Culligan (2015). "Human resistin and the RELM of Inflammation in diabesity." Diabetology \& Metabolic Syndrome 7.

Andersen, G., et al. (2008). "AHSG tag single nucleotide polymorphisms associate with type 2 diabetes and dyslipidemia: studies of metabolic traits in 7,683 white Danish subjects." Diabetes 57(5): 1427-1432.

Bajaj, M. and R. A. Defronzo (2003). "Metabolic and molecular basis of insulin resistance." J Nucl Cardiol 10(3): 311-323.

Banerjee, R. R., et al. (2004). "Regulation of fasted blood glucose by resistin." Science 303(5661): 1195-1198.

Bluher, M. (2014). "Adipokines - removing road blocks to obesity and diabetes therapy." Molecular Metabolism 3(3): 230-240.

Bonner-Weir, S. and G. C. Weir (2005). "New sources of pancreatic beta-cells." Nat Biotechnol 23(7): 857-861. 
Bose, B., et al. (2012). "Human embryonic stem cell differentiation into insulin secreting beta-cells for diabetes." Cell Biol Int 36(11): 1013-1020.

Brandt, C. and B. K. Pedersen (2010). "The role of exercise-induced myokines in muscle homeostasis and the defense against chronic diseases." J Biomed Biotechnol 2010: 520258.

Brown, R., et al. (2007). "Adipokine gene expression in a novel hypothalamic neuronal cell line: resistin-dependent regulation of fasting-induced adipose factor and SOCS-3." Neuroendocrinology 85(4): 232-241.

Buteau, J., (2011). "GLP-1 signaling and the regulation of pancreatic $\beta$-cells mass/function." Avances en Diabetología 27:3-8.

Catoire, M. and S. Kersten (2015). "The search for exercise factors in humans." FASEB J 29(5): 1615-1628.

Chen, S., et al. (2009). "A small molecule that directs differentiation of human ESCs into the pancreatic lineage." Nat Chem Biol 5(4): 258-265.

Chen, Y., et al. (2004). "Retinoic acid signaling is essential for pancreas development and promotes endocrine at the expense of exocrine cell differentiation in Xenopus." Dev Biol 271(1): 144-160.

Choi, K. M. (2016). "The Impact of Organokines on Insulin Resistance, Inflammation, and Atherosclerosis." Endocrinol Metab (Seoul) 31(1): 1-6.

D'Amour, K. A., et al. (2006). "Production of pancreatic hormone-expressing endocrine cells from human embryonic stem cells." Nat Biotechnol 24(11): 1392-1401.

Daquinag, A. C., et al. (2011). "An isoform of decorin is a resistin receptor on the surface of adipose progenitor cells." Cell Stem Cell 9(1): 74-86.

Davila, J. C., et al. (2004). "Use and application of stem cells in toxicology." Toxicological Sciences 79(2): 214-223.

DeFronzo, R.A., et al.(2012) "The role of the kidneys in glucose homeostasis: a new path towards normalizing glycaemia.” Diabetes Obes Metab 2012;14:5-14.

DeFronzo, R.A., et al. (2013). "Characterization of renal glucose reabsorption in response to dapagliflozin in healthy subjects and subjects with type 2 diabetes." Diabetes Care 2013;36:3169-76.

Drucker, D.J,, Nauck, M,A., (2006) “The incretin system: glucagon-like peptide-1 receptor agonists and dipeptidyl peptidase-4 inhibitors in type 2 diabetes." Lancet 368: 1696-1705.

Duff, G. W. and E. Atkins (1982). "The Inhibitory Effect of Polymyxin-B on EndotoxinInduced Endogenous Pyrogen Production." J Immunol Methods 52(3): 333-340.

Eckardt, K., et al. (2014). "Myokines in insulin resistance and type 2 diabetes." Diabetologia 57(6): 1087-1099. 


\section{Chapter 6}

Ertek, S. and A. Cicero (2012). "Impact of physical activity on inflammation: effects on cardiovascular disease risk and other inflammatory conditions." Archives of Medical Science 8(5): 794-804.

Fasshauer, M. and M. Bluher (2015). "Adipokines in health and disease." Trends Pharmacol Sci 36(7): 461-470.

Febbraio, M. A. and B. K. Pedersen (2005). "Contraction,Induced myokine production and release: Is skeletal muscle an endocrine organ.?" Exercise and Sport Sciences Reviews 33(3): 114-119.

Galic, S., et al. (2010). "Adipose tissue as an endocrine organ." Mol Cell Endocrinol 316(2): 129-139.

Gamas, L., et al. (2015). "Irisin and Myonectin Regulation in the Insulin Resistant Muscle: Implications to Adipose Tissue: Muscle Crosstalk." Journal of Diabetes Research.

Ghosh, S., et al. (2003). "The genomic organization of mouse resistin reveals major differences from the human resistin: functional implications." Gene 305(1): 27-34.

Gimeno, R. E. and D. E. Moller (2014). "FGF21-based pharmacotherapy - potential utility for metabolic disorders." Trends in Endocrinology and Metabolism 25(6): 303-311.

Graaf de, C., (2016) "Glucagon-Like Peptide-1 and Its Class B G Protein-Coupled Receptors: A Long March to Therapeutic Successes”. Pharmacol Rev 68(4): 954-1013.

Guan, W. H., et al. (2014). "Genome-Wide Association Study of Plasma N6 Polyunsaturated Fatty Acids Within the Cohorts for Heart and Aging Research in Genomic Epidemiology Consortium." Circulation-Cardiovascular Genetics 7(3): 321-331.

Guo, T. X. and M. Hebrok (2009). "Stem Cells to Pancreatic beta-Cells: New Sources for Diabetes Cell Therapy." Endocr Rev 30(3): 214-227.

Hartman, H. B., et al. (2002). "Mechanisms regulating adipocyte expression of resistin." J Biol Chem 277(22): 19754-19761.

Hartwig, S., et al. (2014). "Secretome profiling Of primary human skeletal muscle cells." Biochimica Et Biophysica Acta-Proteins and Proteomics 1844(5): 1011-1017.

Heinrichsdorff, J. and J. M. Olefsky (2012). "Fetuin-A: the missing link in lipid-induced inflammation." Nat Med 18(8): 1182-1183.

Hiscock, N., et al. (2004). "Skeletal myocytes are a source of interleukin-6 mRNA expression and protein release during contraction: evidence of fiber type specificity." FASEB J 18(9): 992-994.

Holcomb, I. N., et al. (2000). "FIZZ1, a novel cysteine-rich secreted protein associated with pulmonary inflammation, defines a new gene family." Embo Journal 19(15): 4046-4055.

Holst, J.J., Orskov, C. (2004) "The incretin approach for diabetes treatment: modulation of islet hormone release by GLP-1 agonism.” Diabetes. 53 Suppl 3:S197-204.

Hotamisligil, G. S. (2006). "Inflammation and metabolic disorders." Nature 444(7121): 860867. 
Iroz, A., et al. (2015). "Hepatokines: unlocking the multi-organ network in metabolic diseases." Diabetologia 58(8): 1699-1703.

Jabbour, S.A., et al. (2012). "Targeting renal glucose reabsorption for the treatment of type 2 diabetes mellitus using the SGLT2 inhibitor dapagliflozin.” Postgrad Med 124:62-73.

Jensen, J., et al. (2009). "Human embryonic stem cell technologies and drug discovery." J Cell Physiol 219(3): 513-519.

Jiang, W., et al. (2007). "In vitro derivation of functional insulin-producing cells from human embryonic stem cells." Cell Res 17(4): 333-344.

Kawamoto, T., et al. (2008). "TAK-242 selectively suppresses Toll-like receptor 4-signaling mediated by the intracellular domain." Eur J Pharmacol 584(1): 40-48.

Kharitonenkov, A. and A. C. Adams (2014). "Inventing new medicines: The FGF21 story." Molecular Metabolism 3(3): 221-229.

Kraus, D., et al. (2014). "Nicotinamide N-methyltransferase knockdown protects against dietinduced obesity." Nature 508(7495): 258-262.

Kroon, E., et al. (2008). "Pancreatic endoderm derived from human embryonic stem cells generates glucose-responsive insulin-secreting cells in vivo." Nat Biotechnol 26(4): 443452 .

Lazar, M. A. (2007). "Resistin- and Obesity-associated metabolic diseases." Horm Metab Res 39(10): 710-716.

Lee, S., et al. (2014). "Adenylyl Cyclase-Associated Protein 1 Is a Receptor for Human Resistin and Mediates Inflammatory Actions of Human Monocytes." Cell Metab 19(3): 484-497.

Lutz, T.A., Osto, E.(2016) “Glucagon-like peptide-1, glucagon-like peptide-2, and lipid metabolism.” Curr Opin Lipidol 27(3):257-63. doi: 10.1097/MOL.0000000000000293.

Mathews, S. T., et al. (2002). "Improved insulin sensitivity and resistance to weight gain in mice null for the Ahsg gene." Diabetes 51(8): 2450-2458.

McTernan, P. G., et al. (2006). "Resistin." Curr Opin Lipidol 17(2): 170-175.

Medzhitov, R. (2008). "Origin and physiological roles of inflammation." Nature 454(7203): 428-435.

Melone, M., et al. (2012). "Discovery of a New Role of Human Resistin in Hepatocyte LowDensity Lipoprotein Receptor Suppression Mediated in Part by Proprotein Convertase Subtilisin/Kexin Type 9." J Am Coll Cardiol 59(19): 1697-1705.

Mojiminiyi, O. A. and N. A. Abdella (2007). "Associations of resistin with inflammation and insulin resistance in patients with type 2 diabetes mellitus." Scandinavian Journal of Clinical \& Laboratory Investigation 67(2): 215-225.

Molica, F., et al. (2015). "Adipokines at the crossroad between obesity and cardiovascular disease." Thrombosis and Haemostasis 113(3): 553-566. 


\section{Chapter 6}

Monteiro, R. and I. Azevedo (2010). "Chronic Inflammation in Obesity and the Metabolic Syndrome." Mediators of Inflammation.

Moon, B., et al. (2003). "Resistin inhibits glucose uptake in L6 cells independently of changes in insulin signaling and GLUT4 translocation." American Journal of PhysiologyEndocrinology and Metabolism 285(1): E106-E115.

Mukhopadhyay, S. and S. Bhattacharya (2016). "Plasma fetuin-A triggers inflammatory changes in macrophages and adipocytes by acting as an adaptor protein between NEFA and TLR-4." Diabetologia 59(4): 859-860.

Nauck, M. (2016). Incretin therapies : highlighting common features and differences in the modes of action of glucagon-like peptide- 1 receptor agonists and dipeptidyl peptidase-4 inhibitors, 203-216.

Nocon, M., et al. (2008). "Association of physical activity with all-cause and cardiovascular mortality: a systematic review and meta-analysis." European Journal of Cardiovascular Prevention \& Rehabilitation 15(3): 239-246.

Norheim, F., et al. (2011). "Proteomic identification of secreted proteins from human skeletal muscle cells and expression in response to strength training." American Journal of Physiology-Endocrinology and Metabolism 301(5): E1013-E1021.

Owecki, M., et al. (2011). "Serum Resistin Concentrations are Higher in Human Obesity but Independent from Insulin Resistance." Experimental and Clinical Endocrinology \& Diabetes 119(2): 117-121.

Pal, D., et al. (2012). "Fetuin-A acts as an endogenous ligand of TLR4 to promote lipidinduced insulin resistance." Nat Med 18(8): 1279-+.

Park, H. K. and R. S. Ahima (2013). "Resistin in rodents and humans." Diabetes Metab J 37(6): 404-414.

Park, S. E., et al. (2015). "Biomarkers of insulin sensitivity and insulin resistance: Past, present and future." Crit Rev Clin Lab Sci 52(4): 180-190.

Patel, S. D., et al. (2004). "Disulfide-dependent multimeric assembly of resistin family hormones." Science 304(5674): 1154-1158.

Pedersen, B. K. (2011). "Muscles and their myokines." Journal of Experimental Biology 214(2): 337-346.

Pedersen, B. K. and M. A. Febbraio (2007). "Interleukin-6 does/does not have a beneficial role in insulin sensitivity and glucose homeostasis - Point: Interleukin-6 does have a beneficial role in insulin sensitivity and glucose homeostasis." J Appl Physiol (1985) 102(2): 814-816.

Pedersen, B. K. and M. A. Febbraio (2008). "Muscle as an endocrine organ: Focus on musclederived interleukin-6." Physiological Reviews 88(4): 1379-1406. 
Perfetti, R,, et al. (2000). "Glucagon-like peptide-1 induces cell proliferation and pancreaticduodenum homeobox-1 expression and increases endocrine cell mass in the pancreas of old, glucose-intolerant rats." Endocrinology. 141(12):4600-5.

Pratesi, A., et al. (2013). "Skeletal muscle: an endocrine organ." Clin Cases Miner Bone Metab 10(1): 11-14.

Pravenec, M., et al. (2006). "Fat-specific transgenic expression of resistin in the spontaneously hypertensive rat impairs fatty acid re-esterification." Int J Obes (Lond) 30(7): 1157-1159.

Rajala, M. W., et al. (2004). "Regulation of resistin expression and circulating levels in obesity, diabetes, and fasting." Diabetes 53(7): 1671-1679.

Raschke, S. and J. Eckel (2013). "Adipo-Myokines: Two Sides of the Same Coin-Mediators of Inflammation and Mediators of Exercise." Mediators of Inflammation.

Reed J.W., (2016).”Impact of sodium-glucose cotransporter 2 inhibitors on blood pressure.” Vasc Health Risk Manag. 27;12:393-405.

Reilly, M. P., et al. (2005). "Resistin is an inflammatory marker of atherosclerosis in humans." Circulation 111(7): 932-939.

Richardson, T., et al. (2016). "Capsule stiffness regulates the efficiency of pancreatic differentiation of human embryonic stem cells." Acta Biomaterialia 35: 153-165.

Saltiel, A. R. and C. R. Kahn (2001). "Insulin signalling and the regulation of glucose and lipid metabolism." Nature 414(6865): 799-806.

Samuel, V. T. and G. I. Shulman (2012). "Mechanisms for Insulin Resistance: Common Threads and Missing Links." Cell 148(5): 852-871.

Sanchez-Solana, B., et al. (2012). "Mouse Resistin Modulates Adipogenesis and Glucose Uptake in 3T3-L1 Preadipocytes Through the ROR1 Receptor." Molecular Endocrinology 26(1): 110-127.

Sartipy, P., et al. (2007). "The application of human embryonic stem cell technologies to drug discovery." Drug Discovery Today 12(17-18): 688-699.

Scheen A,J., (2016). "DPP-4 inhibitor plus SGLT-2 inhibitor as combination therapy for type 2 diabetes: from rationale to clinical aspects.” Expert Opin Drug Metab Toxicol. 29:1-11.

Schwartz, S. S., \& Ahmed, I. (2016). Sodium - glucose cotransporter 2 inhibitors : an evidence-based practice approach to their use in the natural history of type 2 diabetes, 7995(November). http://doi.org/10.1185/03007995.2016.1151774

Segev, H., et al. (2004). "Differentiation of human embryonic stem cells into insulinproducing clusters." Stem Cells 22(3): 265-274.

Sheng, C. H., et al. (2008). "Resistin is expressed in human hepatocytes and induces insulin resistance." Endocrine 33(2): 135-143. 


\section{Chapter 6}

Siddiq, A., et al. (2005). "A synonymous coding polymorphism in the alpha 2-HeremansSchmid glycoprotein gene is associated with type 2 diabetes in French Caucasians." Diabetes 54(8): 2477-2481.

Stefan, N. and H. U. Haring (2013). "The role of hepatokines in metabolism." Nature Reviews Endocrinology 9(3): 144-152.

Steppan, C. M. and M. A. Lazar (2004). "The current biology of resistin." J Intern Med 255(4): 439-447.

Steppan, C. M., et al. (2005). "Activation of SOCS-3 by resistin." Mol Cell Biol 25(4): 15691575.

Stofkova, A. (2010). "Resistin and visfatin: regulators of insulin sensitivity, inflammation and immunity." Endocr Regul 44(1): 25-36.

Takata, H., et al. (2009). "High Glucose Induces Transactivation of the alpha 2-HS Glycoprotein Gene Through the ERK1/2 Signaling Pathway." Journal of Atherosclerosis and Thrombosis 16(4): 448-456.

Tanaka, T., et al. (2009). "Genome-Wide Association Study of Plasma Polyunsaturated Fatty Acids in the InCHIANTI Study." Plos Genetics 5(1).

Tarkowski, A., et al. (2010). "Resistin competes with lipopolysaccharide for binding to tolllike receptor 4." J Cell Mol Med 14(6B): 1419-1431.

Tenenbaum, A., et al. (2003). "Metabolic syndrome and type 2 diabetes mellitus: focus on peroxisome proliferator activated receptors (PPAR)." Cardiovasc Diabetol 2: 4.

Thomson, J. A. (1998). "Embryonic stem cell lines derived from human blastocysts (vol 282, pg 1147, 1998)." Science 282(5395): 1827-1827.

Tuomilehto, J., et al. (2001). "Prevention of type 2 diabetes mellitus by changes in lifestyle among subjects with impaired glucose tolerance." New England Journal of Medicine 344(18): 1343-1350.

Visintin, A., et al. (2005). "Pharmacological inhibition of endotoxin responses is achieved by targeting the TLR4 coreceptor, MD-2." J Immunol 175(10): 6465-6472.

Wang, H., et al. (2015). "Improvement of Cell Survival During Human Pluripotent Stem Cell Definitive Endoderm Differentiation." Stem Cells and Development 24(21): 2536-2546.

Weir, G. C. and S. BonnerWeir (1997). "Scientific and political impediments to successful islet transplantation." Diabetes 46(8): 1247-1256.

Willson, T. M., et al. (1996). "The structure-activity relationship between peroxisome proliferator-activated receptor gamma agonism and the antihyperglycemic activity of thiazolidinediones." Journal of Medicinal Chemistry 39(3): 665-668.

Yang, R. Z., et al. (2006). "Acute-phase serum amyloid A: An inflammatory adipokine and potential link between obesity and its metabolic complications." Plos Medicine 3(6): 884894. 


\section{Discussion and future perspectives}

Yoo, H. J. and K. M. Choi (2015). "Hepatokines as a Link between Obesity and Cardiovascular Diseases." Diabetes Metab J 39(1): 10-15. 



\section{Chapter 7}

Summary 


\section{Chapter 7}

\section{Summary}

Type 2 Diabetes Mellitus (T2DM) is a modern metabolic disorder. T2DM is related with modern lifestyle and results when the body is unable to produce sufficient insulin or respond properly to insulin, which is the hormone that regulates the levels of glucose in blood. The aim of this project was to define novel targets for the possible development of drugs for T2DM.

Chapter 1 introduces the topic and offers an overview of T2DM and the mechanisms behind it. T2DM results when there is impaired insulin production by beta-cells of the pancreas and from the inability of the peripheral organs to respond properly to insulin by adequate glucose uptake from the blood, reflecting insulin resistance. Insulin sensitizing drugs such as thiazolidinediones (TZDs) and their analogues are currently used for the treatment of T2DM. New directions though are shown since insulin resistance started to be correlated with obesity and inflammation and new targets, like adipokines, might proof to be of value as potential targets for the development of drugs that counteract the effects of T2DM.

In chapter 2 we focused on a possible novel target for insulin therapy which is the adipokine, resistin. It is shown that resistin downregulation in mouse adipocytes would provide an alternative for PPAR $\gamma$ agonist activity, the mode of action of TZDs. To this purpose a reporter gene cell line for PPAR $\gamma$ activation was used and a series of TZDs were tested, obtaining $\mathrm{EC}_{50}$ values for PPAR $\gamma$ activation for each one. The same TZDs were also tested for effects on resistin mRNA expression in adipocytes. The results showed that TZDs with the lowest $\mathrm{EC}_{50}$ for PPAR $\gamma$ activation had also the lowest $\mathrm{IC}_{50}$ for resistin mRNA downregulation. More specifically the results revealed a significant correlation between the $\mathrm{EC}_{50}$ for PPAR $\gamma$ activation in a reporter gene cell line and the $\mathrm{IC}_{50}$ for resistin downregulation in adipocytes. The outcomes indicate that $\operatorname{PPAR} \gamma$ mediated downregulation of resistin might promote insulin sensitivity and that resistin can be used as a target for searching insulin sensitizing compounds.

In chapter 3 we used a commercially available reporter gene cell line (HEK-Blue-TLR-4) to investigate whether detection of TLR-4 mediated gene expression could be a possible bioassay to detect candidate antidiabetic drugs able to inhibit resistin mediated TLR-4 activation and subsequent effects. Firstly it was shown that resistin can activate TLR-4 signaling in the TLR-4 reporter gene assay similar to the natural ligand of TLR-4 which is lipopolysaccharide (LPS). Inhibition of LPS mediated TLR-4 signaling by two known TLR-4 


\section{Summary}

inhibitors could be demonstrated as well as inhibition of resistin mediated TLR-4 signaling by these two known TRL-4 inhibitors. It was concluded that the TLR-4 reporter gene cell line provides a novel tool for detection of resistin antagonists and might be of use as a new target to screen for a possible new type of antidiabetic drugs.

To further increase the possibility for identification of novel targets for antidiabetic drugs via the possible role of resistin, in chapter 4 the mechanism behind resistin mediated effects was investigated. More specifically adipocytes were exposed to resistin and the effects on gene expression were analysed by RNA sequencing. Large sets of genes were upregulated or downregulated. It was observed that resistin dependent differentially expressed genes were involved in downscaling cellular maintenance and cellular processes and in lipid storage. Based on these observations it was proposed that resistin dependent gene expression is related to modifications in cellular maintenance processes, slowing down metabolic changes resulting in energy conservation. Under these circumstances of metabolic adaptation in the presence of resistin, excess food consumption as in modern societies can result in obesity, inflammation and insulin resistance.

In chapter 5 an in vitro model was introduced for the search on novel targets for T2DM that mimics inflamed adipocytes. Adipocytes (3T3-L1) were co-cultured directly with activated macrophages (RAW264.7) producing an environment for testing compounds under conditions more similar to the in vivo situation in T2DM patients. Quantitative mass spectrometry analysis on the co-cultured cells identified clinically known biomarkers, thus confirming the assay. At the same time 54 new proteins that have the potency to be used for research into the mechanism of T2DM were found. Additionally comparison of our results to a study on human visceral fat of obese non-diabetic and obese diabetic patients, indicated that AUH, NAGK, pCYT2, NNMT, STK39 and CSNK2A2 have the potential of being prognostic biomarkers.

Chapter 6 discusses the strategies described in the thesis and gives future perspectives for the detection of novel targets to screen for new drug candidates for the treatment of T2DM. Although T2DM has been studied for a very long period its complexity and systemic nature raises more questions. Altogether it is believed that the strategies presented in this thesis will contribute in answering some of the questions, defining possible novel targets for the development of new drugs candidates for T2DM. 

Acknowledgements

About the author

List of publications

Overview of training activities 


\section{Acknowledgements}

This is the end of a wonderful journey. I would like to express my sincere gratitude to my promotor Ivonne Rietjens and my co-promotor Jacques Vervoort for giving me this great opportunity. I thank you both very much for your support, guidance and trust.

I would not even be in the Netherlands if I did not have the help of professor Konstantinos Zoulfos and professor Ioannis Ambrosiadis. I thank you very much for the first nudge that you gave me for getting into the world of research.

I had a great time at TOX and BIC and I shared wonderful moments with all of you. Therefore I would like to thank all friends and colleagues: Jaime, Henriques, Walter, Ana, Merel, Wasma, Nynke, Samantha, Karsten, Si, Reiko, Erryana, Jonathan, Suzanne, Jochem, Laura, Bert, Hans, Ans, Arif, Sourav, David, Gre, Lidy, Irene, Sjef, Walter, Christof, Christina. And of course my students that helped me. Thank you Marjolein, Corianne, Yijin, Illona, Han and Myrto. My apologies if I forgot someone.

I would like also to thank my project partners Mark, Linda, Richard, Renato and Jean-Paul for all the help they gave me and the constructive meetings we had.

Wageningen has been a meeting point and a place where everybody, including me, could come together with people from all over the world. I grasped this opportunity and met people and made numerous friends that helped me escape the routine. No matter how much I would like to thank each one of you separately, the number of names that I would like to include would not fit in only one or two pages. Thank you all for the memorable times, good and bad (whatever does not kill you, makes you stronger), we had.

My family was always the steadiest mark in my life. A beacon that never stopped guiding me. I thank you so much for the immeasurable help and support that you gave me.

My dear Anastasia. Nothing would be possible without your love, your support and patience. You were my anchor to sanity, and for that I thank you the most. 


\section{About the author}

Alexandros Sotiriou was born on July $3^{\text {rd }} 1980$ in Thessaloniki, Greece. In 1998 he finished secondary school at the Lyceum of Neapoli. In 1999 he succeeded in Panhellenic exams and started his education on Animal production of ATEI of Larisa. In 2003 did his BSc internship at the Veterinary school of Aristotle University in the Laboratory of Food Technology of Animal Origin under the supervision of Prof, Dr. Ioannis Ambrosiadis. In 2005 he decided to move to the Netherlands and follow the MSc programme of Food Safety in Wageningen University. In 2007 after he finished his MSc internship in RIKILT food safety institute under the supervision of Dr. Monique Bremer, he obtained his MSc degree. The same year he worked as a research assistant in the same department in RIKILT. In 2009 he started his PhD work described in this thesis which was a collaboration between the Division of Toxicology, the Laboratory of Biochemistry at Wageningen University, Leadpharma, Modiquest, Chiralix, Biqualis and Hogeschool Utrecht. During his $\mathrm{PhD}$, amongst others he followed the necessary postgraduate courses in toxicology to enable him to register as a European toxicologist. 


\section{List of publications}

Sotiriou A., Blaauw R.H., Meijer C., Gijsbers L.H., van der Burg B., Vervoort J., Rietjens I.M.C.M.. Correlation between activation of PPAR $\gamma$ and resistin downregulation in a mouse adipocyte cell line by a series of thiazolidinediones. Toxicol In Vitro. 2013 Aug;27(5):142532 .

Sotiriou A. Xiong H., De Haan L.H.J., Vervoort J., Rietjens I.M.C.M. Toll-like receptor 4 (TLR-4) signaling as a method for detecting resistin antagonism. Submitted for publication.

Sotiriou A., Gijsbers L., Denissov S., Rietjens I.M.C.M., Musters M., Vervoort J. Resistin exposure of human omental adipocytes affects transcriptional regulation processes involved in cellular differentiation and maintenance. In preparation.

Ten Klooster J.P., Sotiriou A., Boeren S., Vaessen S., Vervoort J., Pieters R. Identification of Type 2 Diabetes-related Biomarkers Derived from an in vitro Model of Inflamed Fat Tissue. Submitted for publication. 


\section{Overview of training activities}

\section{Discipline specific courses}

Toxicogenomics

Molecular Toxicology

Risk Assessment

Proteomics

Organ Toxicology

Mutagenesis and Carcinogenesis

Pathobiology

Immunotoxicology

Medical and Forensic Toxicology

Legal and Regulatory Toxicology

\section{Attended conferences}

Annual meeting of the NVT

Annual meeting of the NVT

SOT annual meeting and ToxExpo

\section{General courses}

VLAG PhD week

Coaching for Personal Effectiveness

Lab. Animal Science (B)

\section{Optional activities}

Preparation of $\mathrm{PhD}$ research proposal

Attending scientific presentations and discussions

General Toxicology

Ecotoxicology
Zeist, the Netherlands 2010

Zeist, the Netherlands 2011

San Antonio, USA 2013

$\begin{array}{ll}\text { PET } & 2010 \\ \text { PET } & 2011 \\ \text { VLAG } & 2011 \\ \text { PET } & 2011 \\ \text { PET } & 2011 \\ \text { PET } & 2012 \\ \text { PET } & 2012 \\ \text { PET } & 2012 \\ \text { PET } & 2012\end{array}$

VLAG 2010

Meijer and Meijaard 2011

PET

2012
TOX-BIC 2009

TOX-BIC 2009-2013

TOX 2013

TOX 
The research described in this thesis was financially supported by the Dutch Ministry of Economic Affairs, Agriculture and Innovation, Grant Pieken in de Delta - Healthy ageing project, PID082022.

Cover design: D. Sotiriou

Printed by: Ridderprint, Ridderkerk (NL). Ridderprint.nl 